\title{
Pfaffians, the $G$-signature theorem and Galois Hodge discriminants
}

\author{
Ted Chinburg, Georgios Pappas and Martin Taylor
}

\begin{abstract}
Let $G$ be a finite group acting freely on a smooth projective scheme $X$ over a locally compact field of characteristic 0 . We show that the $\varepsilon_{0}$-constants associated to symplectic representations $V$ of $G$ and the action of $G$ on $X$ may be determined from Pfaffian invariants associated to duality pairings on Hodge cohomology. We also use such Pfaffian invariants, along with equivariant Arakelov Euler characteristics, to determine hermitian Euler characteristics associated to tame actions of finite groups on regular projective schemes over $\mathbb{Z}$.
\end{abstract}

\section{Introduction}

Suppose $F$ is a locally compact field of characteristic 0 and that $X$ is a smooth projective scheme over $F$ which is equidimensional of dimension $d$ and which has a free action by a finite group $G$. Deligne's theory of local constants associates to each complex representation $V$ of $G$ an $\varepsilon_{0}$-constant $\varepsilon_{0}(X, V)$ depending on some additional choices which enters into the theory of functional equations of L-series. The object of this paper is to give a characterization of $\varepsilon_{0}(X, V)$ when $V$ is symplectic in terms of invariants associated to the duality pairings on Hodge cohomology. If $F$ is archimedean, the Hodge cohomology in question is that of $X$. If $F$ is non-archimedean with ring of integers $O_{F}$, we must assume that there is a regular projective scheme $\mathcal{X}$ over $O_{F}$ having a tame action of $G$ and general fiber the $G$-scheme $X$. The Hodge cohomology one takes is then that of $\mathcal{X}$.

The invariants we study arise from Pfaffians of the $V$-isotypic components of duality pairings on Hodge cohomology. Pfaffians are associated to alternating non-degenerate bilinear forms $\langle$,$\rangle on a$ finite-dimensional $F$-vector space $W$ of dimension $2 n$ over $F$. Classically these provide a square root of the discriminant of the form. We define the Pfaffian of $\langle$,$\rangle to be the unique linear functional$

$$
\text { Pf }: \operatorname{det}(W)=\operatorname{det}(U) \otimes \operatorname{det}(W / U) \rightarrow F
$$

with the following property. Let $U$ be a maximal isotropic subspace of $W$, and identify $U^{D}=$ $\operatorname{Hom}_{F}(U, F)$ with $W / U$ via $\langle$,$\rangle . We define Pf to be the natural contraction functional \operatorname{det}(U) \otimes$ $\operatorname{det}\left(U^{D}\right) \rightarrow F$ (see $\S \S 2.1$ and 2.2 ). It is not difficult to extend this to complexes; see $\S 2.3$.

If $F$ is archimedean, the epsilon constant $\varepsilon_{0}(X, V)$ is positive if $F=\mathbb{C}$, so suppose that $F=\mathbb{R}$ and that $V$ is a complex symplectic representation. The domain of the Pfaffian functional associated to the $V$-isotypic component of the Hodge cohomology of $X$ is a one-dimensional $\mathbb{C}$-vector space $L_{V}$. Because the action of $G$ on $X$ is free, the Hodge cohomology of $X$ can be computed by a perfect complex of $\mathbb{R}[G]$-modules. This leads to an $\mathbb{R}$-line in $L_{V}$ as well as a notion of positivity in this line.

Received 1 December 2003, accepted 2 August 2006, final version received 14 November 2006. 2000 Mathematics Subject Classification 14L30 (primary), 14F10, 11G40, 14C30 (secondary).

Keywords: Hodge cohomology, duality pairings, local constants, Pfaffians.

The first author was supported by NSF Grants DMS00-70433 and DMS-0500106. The second author was supported by NSF Grant DMS05-01409. The third author was supported by an EPSRC Senior Research Fellowship and a Royal Society Wolfson Merit award.

This journal is (C) Foundation Compositio Mathematica 2007. 


\section{T. Chinburg, G. Pappas and M. Taylor}

Our main result shows that, if $V$ is a virtual symplectic representation of dimension 0 , the sign of $\varepsilon_{0}(X, V)$ is the sign of the image of a positive real generator of $L_{V}$ under the Pfaffian functional. The essential ingredient needed to capture this sign information is the $G$-signature theorem.

Suppose now that $F$ is non-archimedean of residue characteristic $p$, and let $\mathcal{X}$ be a model of $X$ over $O_{F}$ as above. Let $V$ be a complex virtual symplectic representation of dimension 0 . The model $\mathcal{X}$ leads to an $O_{F}$-line inside $L_{V}$. We show that the valuation of the Pfaffian on a generator of this line gives the valuation $\varepsilon_{0}(X, V)$. The key ingredient needed to prove this is the characterization in [CEPT98] of $\varepsilon_{0}$-constants in terms of intersection numbers of suitable Pfaffian divisors. We show that the above valuation information actually determines $\varepsilon_{0}(X, V)$, in the following way. By work of Saito [Sai93] and Cassou-Noguès and Taylor [CNT83], the function which sends each symplectic $V$ of dimension 0 to $\varepsilon_{0}(X, V)$ lies in a subgroup $M$ of 'rational classes' in the group $\operatorname{Hom}_{\mathrm{Gal}(\overline{\mathbb{Q}} / \mathbb{Q})}\left(R_{G, 0}^{\mathrm{s}}, \mathbb{Q}^{*}\right)$, where $R_{G, 0}^{\mathrm{s}}$ is the group of symplectic characters of dimension 0 . The results in [CNT83] show that each $f \in M$ is determined by the $p$-adic valuations of its values.

These results generalize to $X$ of arbitrary dimension the results in [CPT03], and they refine the results in [CPT02] concerning equivariant Arakelov Euler characteristics associated to $G$-schemes which are projective over $\mathbb{Z}$. In [CPT02] we considered the Quillen metrics at archimedean places on the determinants of the isotypic components of de Rham cohomology. This leads to studying invariants in a suitable equivariant Arakelov class group and their relation to $\varepsilon$-constants. The results in this paper concern Pfaffians of duality pairings, and the natural algebraic invariants lie in adelic hermitian class groups of the finite group $G$. These hermitian invariants refine the previous Arakelov Euler characteristics, in that they encode sign information. To relate the hermitian invariants with the Arakelov invariants we make use of a variant due to Maillot and Roessler [MR04, Lemma 2.8] of a result of Ray and Singer [RS73] on the vanishing of analytic torsion.

We now state more precisely our main result when $F=\mathbb{R}$. In $\S 3.1$ we recall from [CPT03] the symmetric $G$-invariant pairings on Hodge cohomology

$$
\sigma_{X}^{t}: \mathrm{H}^{t}\left(R \Gamma\left(X, \bigoplus_{i=0}^{d} \Omega_{X / \mathbb{R}}^{i}[d-i]\right)\right) \times \mathrm{H}^{-t}\left(R \Gamma\left(X, \bigoplus_{i=0}^{d} \Omega_{X / \mathbb{R}}^{i}[d-i]\right)\right) \rightarrow \mathbb{R}
$$

which arise from Serre duality. For each symplectic character $\theta$ of $G$, these pairings, together with the Pfaffian construction, determine the Pfaffian linear functional on the $\theta$-component of the equivariant determinant of cohomology of $R \Gamma\left(X, \bigoplus_{i} \Omega_{X / \mathbb{R}}^{i}[d-i]\right)$. From the work in $\S 2.5$ it will follow that, on a certain family of distinguished sections of the symplectic components of the determinant of cohomology, the Pfaffian functional takes real values whose signs are independent of choices. For a given symplectic character $\theta$ of $G$ we shall denote this sign invariant by

$$
\text { sgn.pf }\left(\theta, \sigma, R \Gamma\left(X, \bigoplus_{i=0}^{d} \Omega_{X / \mathbb{R}}^{i}[d-i]\right)\right) \text {. }
$$

Let $V_{0}=\mathrm{H}^{0}\left(R \Gamma\left(X, \bigoplus_{i=0}^{d} \Omega_{X / \mathbb{R}}^{i}[d-i]\right)\right)$ and let $V_{t}=\bigoplus_{s= \pm t} \mathrm{H}^{\mathrm{s}}\left(R \Gamma\left(X, \bigoplus_{i=0}^{d} \Omega_{X / \mathbb{R}}^{i}[d-i]\right)\right)$ for $t>0$. Then $\sigma_{X}^{0}$ (respectively $\sigma_{X}^{t} \oplus \sigma_{X}^{-t}$ ) gives a pairing on $V_{t}$ if $t=0$ (respectively if $t>0$ ), and we define $V_{t}^{-}$to be the maximal $\mathbb{R}[G]$-submodule of $V_{t}$ on which this pairing is negative definite. Let $n_{\theta}^{-}(\sigma)$ denote the sum over $t \geqslant 0$ of $(-1)^{t}$ times the usual inner product of the character of $V_{t}^{-}$ as an $\mathbb{R}[G]$-module with the (real-valued) character $\theta$, where the irreducible complex characters are orthonormal with respect to this inner product. For further details, see $\S \S 2.6$ and 3.1.

Let $Z$ be a compact oriented real manifold of even dimension $2 d$ on which $G$ acts. Define $\mathrm{H}_{B}^{d}(Z, \mathbb{R})^{ \pm}$to be a maximal $\mathbb{R}[G]$-submodule of the Betti cohomology group $\mathrm{H}_{B}^{d}(Z, \mathbb{R})$ on which the cup-product form is positive definite, respectively negative definite. Virtual modules $\mathrm{H}_{B}^{\bullet}(Z, \mathbb{R})^{ \pm}$ are defined similarly by extending the cup-product form to all the Betti cohomology groups of $Z$ 


\section{PfaffiAns AND HodGe DISCRIMINANTS}

(see $\S 3.3$ for details). We let $\chi^{ \pm}(Z)$ denote the dimension of $\mathrm{H}_{B}^{\bullet}(Z, \mathbb{R})^{ \pm}$, so that $\chi^{+}(Z)+\chi^{-}(Z)$ is the Euler characteristic $\chi(Z)$. We define

$$
\delta(Z)= \begin{cases}\chi(Z) / 2 & \text { if } d \text { is odd } \\ \chi^{+}(Z) & \text { if } d \equiv 2 \bmod 4 \\ \chi^{-}(Z) & \text { if } d \equiv 0 \bmod 4\end{cases}
$$

Theorem 1.1. Suppose that $F=\mathbb{R}$ and that $\theta$ is a symplectic character of $G$. Let $Y$ be the (smooth projective) quotient scheme $X / G$, and define $\delta(Y)=\delta(Y(\mathbb{C}))$. Then

$$
\text { sgn.pf }\left(\theta, \sigma, R \Gamma\left(\bigoplus_{i=0}^{d} \Omega_{X / \mathbb{R}}^{i}[d-i]\right)\right)=(\sqrt{-1})^{n_{\theta}^{-}(\sigma)}=(-1)^{\delta(Y) \theta(1) / 2} \varepsilon_{0}(X, \theta),
$$

where $\varepsilon_{0}(X, \theta)$ is the archimedean local constant described in the first part of the Introduction.

The proof of this archimedean result has the following ingredients. The first is that $\varepsilon_{0}$-constants of virtual symplectic representations of dimension 0 can be computed from the dimensions of the eigenspaces under complex conjugation of the $G$-isotypic pieces of the Betti cohomology of $X(\mathbb{C})$. Our strategy is to compute these dimensions, which for simplicity we will call Betti conjugation dimensions, via the dimensions of the positive and negative definite parts of the $G$-isotypic pieces of the de Rham cohomology of $X$ under the de Rham duality pairings, which we will call the de Rham pairing dimensions. To accomplish this, we need the Atiyah-Singer signature theorem, which shows that both the positive and the negative definite parts of the total Betti cohomology with respect to the natural cup-product pairing are free $\mathbb{R}[G]$-modules. Furthermore, one has a $G$-equivariant comparison isomorphism between Betti and de Rham cohomology. A key algebraic result (Proposition 2.15) shows how to use the comparison isomorphism to compute the Betti conjugation dimensions entering into the $\varepsilon_{0}$-constants in terms of the de Rham duality pairing dimensions. The latter dimensions are computed by comparing the duality pairings on de Rham and Hodge cohomology and by then using equivariant Pfaffians associated to Hodge cohomology. This leads to Theorem 1.1.

We now state more precisely our result about $\varepsilon_{0}$-constants when $F$ is a non-archimedean local field of characteristic 0 and residue characteristic $p>0$. Suppose as before that there is a regular flat projective model $\mathcal{X}$ of $X$ over the integers $O_{F}$ of $F$ on which $G$ acts tamely. We will also assume that both $\mathcal{X}$ and the quotient $\mathcal{Y}=\mathcal{X} / G$ are regular, and that the special fibers $\mathcal{X}_{p}^{\text {red }}$ and $\mathcal{Y}_{p}^{\text {red }}$ are divisors with normal crossings and multiplicities prime to $p$. Let $\mathbb{F}_{q}$ be the residue field of $O_{F}$, and let $\Omega_{\mathcal{X} / O_{F}}^{i}\left(\log \mathcal{X}_{p}^{\text {red }} / \log \mathbb{F}_{q}\right)$ be the sheaf of relative logarithmic differential $i$-forms on $\mathcal{X}$. Let $\theta$ be the character of a symplectic representation of $G$ which is realized over a finite extension $N$ of $F$. The Pfaffian construction applied to the pairings defined by Serre duality determine a Pfaffian linear functional

$$
\operatorname{Pf}_{\theta}: \operatorname{det}\left(R \Gamma\left(X, \bigoplus_{i=0}^{d} \Omega_{X / F}^{i}[d-i]\right)\right)_{\theta} \rightarrow N
$$

on the $\theta$-component of the equivariant determinant of cohomology of $R \Gamma\left(X, \bigoplus_{i} \Omega_{X / F}^{i}[d-i]\right)$. Let $|\operatorname{Pf}(\mathcal{X}, \theta)|_{p}$ be the $p$-adic absolute value of the image under $\operatorname{Pf}_{\theta}$ of any generator for the $O_{N}$-line $\operatorname{det}\left(R \Gamma\left(\mathcal{X}, \bigoplus_{i=0}^{d} \Omega_{\mathcal{X} / O_{F}}^{i}\left(\log \mathcal{X}_{p}^{\text {red }} / \log \mathbb{F}_{q}\right)\right)[d-i]\right)_{\theta}$.

Theorem 1.2. Suppose $p$ does not divide \#G. The constant

$$
\tilde{\varepsilon}_{0}(\theta)=\varepsilon_{0}\left(\theta-\operatorname{dim}(\theta) \cdot 1_{G}\right)
$$

lies in $\pm p^{\mathbb{Z}}$ and

$$
\left|\operatorname{Pf}\left(\mathcal{X}, \theta-\operatorname{dim}(\theta) \cdot 1_{G}\right)\right|_{p}^{(-1)^{d}}=\left|\tilde{\varepsilon}_{0}(\theta)\right|_{p}
$$




\section{T. Chinburg, G. Pappas and M. Taylor}

These $p$-adic absolute values as $\theta$ varies over all symplectic representations of $G$ determine the sign of $\tilde{\varepsilon}_{0}(\theta)$ for all such $\theta$.

The first ingredient in proving this theorem is a result in [CEPT98] showing that the valuations of $\varepsilon_{0}$-constants of virtual symplectic representations of dimension 0 are equal to the intersection numbers of certain Pfaffian divisors on the integral model $\mathcal{X}$ of $X$ with the top Chern class of the relative logarithmic differentials on $\mathcal{X}$. Twice the valuation of the Pfaffian evaluated on an integral generator is a discriminant of the duality pairing on the logarithmic differentials. This can be computed by a localized Riemann-Roch theorem and agrees with twice the desired intersection number. This leads to our result concerning the valuation of $\tilde{\varepsilon}_{0}(\theta)$. The fact that $\tilde{\varepsilon}_{0}(\theta)$ is determined by these valuations is a consequence of an algebraic result of Cassou-Noguès and Taylor in [CNT83] concerning 'rational classes' in the adelic hermitian class group of $G$. One can view this result as saying that, as $\theta$ varies, the $\tilde{\varepsilon}_{0}(\theta)$ satisfy sufficiently many congruences at primes $l \mid \# G$ to be able to deduce their signs from their $p$-adic absolute values.

Our final result compares certain hermitian Euler characteristics constructed by Pfaffian invariants to the equivariant Arakelov Euler characteristics considered in [CPT02]. We now suppose that $\mathcal{X}$ is a regular flat projective scheme over $\mathbb{Z}$ on which $G$ acts tamely. We will also assume that both $\mathcal{X}$ and the quotient $\mathcal{Y}=\mathcal{X} / G$ is regular, with special fibers which are divisors with normal crossings and multiplicities prime to the residue characteristic. Since $\mathcal{X}$ is regular, we may choose a resolution of $\Omega_{\mathcal{X} / \mathbb{Z}}^{1}$ by a length 2 complex $K^{\bullet}$ of $G$-equivariant locally free $O_{\mathcal{X}}$-sheaves. For $i \geqslant 0$ and we let $L \wedge^{i}$ denote the $i$ th left derived exterior power functor of Dold and Puppe [DP61] on perfect complexes of $G$-equivariant $O_{\mathcal{X}}$-sheaves (that is to say, $O_{\mathcal{X}}$-sheaves with a $G$-action which is compatible with the $G$-action on $O_{\mathcal{X}}$ ). Thus $L \wedge^{i} K^{\bullet}$ denotes the complex arising from the application of $L \wedge^{i}$ to $K^{\bullet}$ and we define $L \wedge^{\bullet} \Omega_{\mathcal{X} / \mathbb{Z}}^{1}$ to be the direct sum of the complexes $L \wedge^{i} K^{\bullet}[-i]$ for $0 \leqslant i \leqslant d$. (For further details, see $\S 6$.)

In $\S 5.1$, we recall the definition of the hermitian class group $\mathrm{H}^{\mathrm{s}}(\mathbb{Z}[G])$, the Arakelov class group $\mathrm{A}(\mathbb{Z}[G])$ and the symplectic Arakelov class group $\mathrm{A}^{\mathrm{s}}(\mathbb{Z}[G])$. In $\S \S 5.3$ and 6 , we use Pfaffians of the pairings $\sigma_{X}$ on Hodge cohomology of the general fiber $X$ to define a hermitian Euler characteristic $\chi_{\mathrm{H}}^{\mathrm{s}}\left(R \Gamma\left(\mathcal{X}, L \wedge^{\bullet} \Omega_{\mathcal{X} / \mathbb{Z}}^{1}\right), \sigma_{X}\right)$ in $\mathrm{H}^{\mathrm{s}}(\mathbb{Z}[G])$. In [CPT02] we considered the so-called equivariant Arakelov class $\chi_{A, \mathcal{X}}$ in $\mathrm{A}(\mathbb{Z}[G])$ obtained by endowing the equivariant determinant of cohomology of $R \Gamma\left(\mathcal{X}, L \wedge \bullet \Omega_{\mathcal{X} / \mathbb{Z}}^{1}\right)$ with certain Quillen metrics. In $\S 5.2$ we shall show that an arbitrary hermitian Euler characteristic admits a natural decomposition into the product of a symplectic Arakelov class and a signature invariant, i.e. that

$$
\mathrm{H}^{\mathrm{s}}(\mathbb{Z}[G])=\mathrm{A}^{\mathrm{s}}(\mathbb{Z}[G]) \times \mathrm{S}_{\infty}(\mathbb{Z}[G])
$$

where $\mathrm{S}_{\infty}(\mathbb{Z}[G])$ is isomorphic to $\operatorname{Hom}\left(R_{G}^{\mathrm{s}}, \pm 1\right)$ when $R_{G}^{\mathrm{s}}$ is the group of symplectic characters of $G$.

TheOREm 1.3. With the above notation and hypotheses, the hermitian Euler characteristic $\chi_{\mathrm{H}}^{\mathrm{s}}$ $\left(R \Gamma\left(\mathcal{X}, L \wedge \bullet \Omega_{\mathcal{X} / \mathbb{Z}}^{1}\right), \sigma\right)$ is equal to

$$
\chi_{A, \mathcal{X}}^{\mathrm{s}} \times \operatorname{sgn} \cdot \operatorname{pf}\left(\sigma, R \Gamma\left(L \wedge^{\bullet} \Omega_{X / \mathbb{Q}}^{1}\right)\right)
$$

relative to the decomposition in (1.1), where $\chi_{A, \mathcal{X}}^{\mathrm{s}}$ is the class in $A^{\mathrm{s}}(\mathbb{Z}[G])$ obtained by restricting $\chi_{A, \mathcal{X}}$ to symplectic characters.

The proof of this theorem depends crucially on a generalization of a result of Ray and Singer [RS73] due to Maillot and Roessler [MR04], which enables us to show that the equivariant analytic torsion for the de Rham complex vanishes. It is this that allows us to relate the Arakelov invariant $\chi_{A, \mathcal{X}}$, defined via Quillen metrics, to the hermitian Hodge Euler characteristic defined by duality parings. 


\section{Pfaffians AND Hodge DiscRiminants}

The terms of (1.2) admit the following numerical interpretation. The second term of (1.2) is determined in terms of archimedean $\varepsilon$-constants by Theorem 1.1. In [CPT02] the first term $\chi_{A, \mathcal{X}}^{\mathrm{s}}$ was shown to lie in a group of 'rational classes' isomorphic to $\operatorname{Hom}_{\mathrm{Gal}(\overline{\mathbb{Q}} / \mathbb{Q})}\left(R_{G}^{\mathrm{s}}, \mathbb{Q}^{\times}\right)$, and the function representing $\chi_{A, \mathcal{X}}^{\mathrm{s}}$ in this group was shown to be the product of the character function $\theta \mapsto \varepsilon(\mathcal{Y}, \theta)$ with an elementary ramification function (see [CPT02, Theorem 1] for details). It is interesting to note that we have two natural sign invariants. The first sign invariant is given by the signs of the nonzero rational numbers obtained by identifying $\chi_{A, \mathcal{X}}^{\mathrm{s}}$ with an element of $\operatorname{Hom}_{\mathrm{Gal}(\overline{\mathbb{Q}} / \mathbb{Q})}\left(R_{G}^{\mathrm{s}}, \mathbb{Q}^{\times}\right)$. The second sign invariant should be thought of as the archimedean signature. Such a double appearance of sign invariants was apparent in the work of Fröhlich (see for instance [Frö84, Corollary 3, p. 192]).

We now explain the structure of the paper.

In $\S 2$ we discuss generalities about Pfaffians and their equivariant generalization to complexes on which one has non-degenerate equivariant symmetric forms. In $\S 3$, we compare duality pairings on Hodge, de Rham, Betti and Dolbeault cohomology in order to relate their positive definite and negative definite subspaces. In $\S 4$ we prove Theorem 1.1. In $\S 5$ we recall the definitions of various class groups needed to describe the hermitian and Arakelov invariants considered in $\S 6$. The proof of Theorem 1.3 is completed in $\S 6.3$. The non-archimedean result Theorem 1.2 is proved in $\S 7$. In Appendix A we compare the definition of the hermitian class group used in $\S 5$ with the one used in [Frö84] and in [CPT03].

\section{Pfaffians}

In this section we work over an arbitrary field $K$ of characteristic 0 . All vector spaces are assumed to be finite-dimensional and all bilinear forms are assumed to be non-degenerate. In this section we present the basic theory of Pfaffians that we shall require for our applications in the remainder of this paper.

\subsection{Determinants}

For a $K$-vector space $V$ we let $V^{D}$ denote the $K$-linear dual $\operatorname{Hom}_{K}(V, K)$, and if $V$ has dimension $d$, we write $\operatorname{det}(V)=\wedge^{d} V$. When $V$ is a line we usually write $V^{-1}$ for $V^{D}$.

Throughout this paper we shall adopt the following convention of Deligne concerning determinants. Let $W$ be a vector space over $K$, and suppose $U$ and $V$ are subspaces of $W$ which span $W$ and have intersection $\{0\}$. We will use the Koszul isomorphism

$$
\operatorname{det}(U) \otimes \operatorname{det}(V) \cong \operatorname{det}(V) \otimes \operatorname{det}(U) \quad \text { defined by } \quad \alpha \otimes \beta \rightarrow(-1)^{\operatorname{dim}(U) \operatorname{dim}(V)} \beta \otimes \alpha .
$$

This is the isomorphism which results from the isomorphisms $\operatorname{det}(U) \otimes \operatorname{det}(V) \rightarrow \operatorname{det}(W)$ and $\operatorname{det}(V) \otimes \operatorname{det}(U) \rightarrow \operatorname{det}(W)$ induced by the inclusions of $U$ and $V$ into $W$. In most of our calculations, the dimension of at least one of the terms $\operatorname{dim}(V)$ and $\operatorname{dim}(W)$ will be even, so we will not have to keep track of Koszul rule sign changes.

We will identify $\operatorname{det}\left(V^{D}\right)$ with $\operatorname{det}(V)^{D}$ using the isomorphism $h_{V}: \operatorname{det}(V) \otimes \operatorname{det}\left(V^{D}\right) \rightarrow K$ for which

$$
\left(v_{1} \wedge \cdots \wedge v_{n}\right) \otimes\left(v_{n}^{*} \wedge \cdots \wedge v_{1}^{*}\right) \rightarrow 1
$$

if $\left\{v_{k}^{*}\right\}_{k}$ is a dual basis to $\left\{v_{j}\right\}_{j}$. This identification is compatible with direct sums when one uses the above Koszul rule to identify

$$
\operatorname{det}(U \oplus V) \otimes_{K} \operatorname{det}\left(U^{D} \oplus V^{D}\right)=\operatorname{det}(U) \otimes \operatorname{det}(V) \otimes \operatorname{det}\left(U^{D}\right) \otimes \operatorname{det}\left(V^{D}\right)
$$

with $\operatorname{det}(U) \otimes \operatorname{det}\left(U^{D}\right) \otimes \operatorname{det}(V) \otimes \operatorname{det}\left(V^{D}\right)$. 


\section{T. Chinburg, G. Pappas and M. Taylor}

Finally, if $n=\operatorname{dim}_{K}(V)$ is even and $m=\operatorname{dim}_{K}(U)$, we fix an identification

$$
\operatorname{det}\left(U \otimes_{K} V\right)=\operatorname{det}(U)^{\otimes n} \otimes \operatorname{det}(V)^{\otimes m}
$$

by sending $\bigwedge_{i, j}\left(u_{i} \otimes v_{j}\right)$ to $\left(\bigwedge_{i} u_{i}\right)^{\otimes n} \otimes\left(\bigwedge_{j} v_{j}\right)^{\otimes m}$ for each ordered basis $\left\{u_{i}\right\}_{i}$ (respectively $\left.\left\{v_{j}\right\}_{j}\right)$ of $U$ (respectively $V$ ), where we give $\left\{u_{k} \otimes v_{j}\right\}_{i, j}$ the lexicographic ordering.

\subsection{Pfaffians of vector spaces}

Our basic references for the theory of Pfaffians are [Frö84, § II.3] and [Lan84, ch. XIV]. We begin by recalling the notion of discriminant for a non-degenerate bilinear form $h$ on $V$. Thus such a form $h$ affords an isomorphism $h: V \rightarrow V^{D}$, via the rule $h(x)(y)=h(y, x)$. The discriminant $d_{h}$ is then defined to be the linear isomorphism of one-dimensional $K$-vector spaces

$$
d_{h}: \operatorname{det}(V)^{\otimes 2} \underset{1 \otimes \operatorname{det}(h)}{\longrightarrow} \operatorname{det}(V) \otimes \operatorname{det}\left(V^{D}\right) \rightarrow K
$$

given by using the above isomorphism $\operatorname{det}\left(V^{D}\right) \cong \operatorname{det}(V)^{D}$ and contraction.

Suppose now that $h$ is an alternating form. Since $h$ is assumed to be non-degenerate, [Lan84, $\S$ XIV.9] shows that $\operatorname{dim}(V)=2 n$ and that all maximal isotropic subspaces $U$ of $V$ with respect to $h$ have dimension $n$. The form $h$ gives an isomorphism $h: V / U \rightarrow U^{D}$ via the rule $h(x \bmod U)(y)=$ $h(y, x)$. We have an isomorphism

$$
\operatorname{det}(V)=\operatorname{det}(U) \otimes \operatorname{det}(V / U)
$$

which sends $\alpha \wedge \beta$ to $\alpha \otimes \bar{\beta}$ for $\alpha \in \operatorname{det}(U)$ and $\beta \in \wedge^{n}(V)$, where $\bar{\beta}$ is the image of $\beta$ in $\operatorname{det}(V / U)$. We define

$$
\text { Pf }: \operatorname{det}(V) \rightarrow K
$$

to be the composition of (2.2) with the isomorphism

$$
\operatorname{det}(U) \otimes \operatorname{det}(V / U)=\operatorname{det}(U) \otimes \operatorname{det}\left(U^{D}\right)
$$

induced by $h: V / U \rightarrow U^{D}$ followed by the map $\operatorname{det}(U) \otimes \operatorname{det}\left(U^{D}\right)=\operatorname{det}(U) \otimes \operatorname{det}(U)^{D} \rightarrow K$ induced by our identification of $\operatorname{det}\left(U^{D}\right)$ with $\operatorname{det}(U)^{D}$ followed by contraction.

Suppose $U^{\prime}$ is another maximal isotropic subspace of $V$. By [Lan84, $\S$ XIV.9] there is an automorphism $\alpha: V \rightarrow V$ which preserves the form $h$ and carries $U$ to $U^{\prime}$. The isomorphism $V / U \rightarrow V / U^{\prime}$ induced by $v \rightarrow \alpha(v)$ is then identified with the inverse of the isomorphism $U^{\prime D} \rightarrow U^{D}$ induced by $\alpha: U \rightarrow U^{\prime}$. It follows that to prove that $\mathrm{Pf}$ is independent of the choice of $U$, it suffices to show that $\operatorname{det}(\alpha)=1$. One way to prove this well-known fact is to pass to the algebraic closure of $K$ and to use eigenvectors of $\alpha$ to show that there is a maximal isotropic subspace $U^{\prime \prime}$ of $V$ which is stable under $\alpha$. This leads to $\operatorname{det}(\alpha)=1$ on $\operatorname{det}(V)=\operatorname{det}\left(U^{\prime \prime}\right) \otimes \operatorname{det}\left(V / U^{\prime \prime}\right)=\operatorname{det}\left(U^{\prime \prime}\right) \otimes \operatorname{det}\left(U^{\prime \prime D}\right)$ since $\alpha$ preserves $h$.

We now briefly recall some elementary properties of Pfaffians.

The map

$$
\mathrm{Pf}_{h}^{\otimes 2}: \operatorname{det}(V)^{\otimes 2} \rightarrow K
$$

induced by the product map $K \otimes K \rightarrow K$ is $(-1)^{n} \cdot d_{h}$.

For $i=1,2$, let $h_{i}$ be an alternating form on the vector space $V_{i}$. Let $h_{1} \oplus h_{2}$ denote the orthogonal sum form on $V_{1} \oplus V_{2}$. Then, from the definition of $\mathrm{Pf}_{h}$, we see that $\mathrm{Pf}_{h_{1} \oplus h_{2}}=\operatorname{Pf}_{h_{1}} \otimes \mathrm{Pf}_{h_{2}}$ under the identification $\operatorname{det}\left(V_{1} \oplus V_{2}\right)=\operatorname{det}\left(V_{1}\right) \otimes \operatorname{det}\left(V_{2}\right)$.

For an alternating form $h$ on $V$ and for a given isomorphism of $K$-vector spaces $\phi: V \rightarrow W$, let $\phi_{*} h$ denote the form on $W$ given by the rule $\phi_{*} h(x, y)=h\left(\phi^{-1} x, \phi^{-1} y\right)$. Then, since $\phi$ maps a maximal isotropic subspace of $V$ with respect to $h$ to such a subspace for $W$ with respect to $\phi_{*} h$, 


\section{Pfaffians AND Hodge DiscRiminants}

the following diagram commutes.

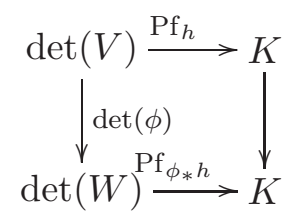

Proposition 2.1. For a given alternating form $h$ on $V$ and for an automorphism $A$ of $V$, let $\widehat{A}$ denote the adjoint of $A$ with respect to $h$; that is to say, $h(A x, y)=h(x, \widehat{A} y)$. Suppose $A$ is selfadjoint, so that $A=\widehat{A}$, and define $h^{\prime}(x, y)=h(A x, y)$. Then there is an automorphism $B$ of $V$ such that $h=B_{*} h^{\prime}$. This implies that $A=\widehat{B} B$. By (2.3), the value $\operatorname{det}(B) \operatorname{depends}$ only on $A$ and we call it the Pfaffian of $A$, denoted $\mathbf{p f}_{h}(A)$. Thus

$$
\mathrm{Pf}_{h^{\prime}}=\mathbf{p f}_{h}(A) \mathrm{Pf}_{h} \text { and } \mathbf{p f}_{h}(A)^{2}=\operatorname{det}(A) .
$$

Remark 2.2. In the sequel Pf will denote a functional on a $K$-line, whereas pf will denote the Pfaffian of an automorphism. Note that if $c$ is a non-zero scalar and $c h$ is the form defined by $(c h)(x, y)=c h(x, y)$, then $\mathbf{p} \mathbf{f}_{c h}(A)=\mathbf{p f}_{h}(A)$ since $A$ has the same adjoint with respect to $h$ and $c h$.

\subsection{Pfaffians of complexes}

Let $C^{\bullet}$ denote a bounded complex of vector spaces over a field $K$. We put

$$
C^{\mathrm{ev}}=C^{0} \bigoplus_{i>0}\left(C^{2 i} \oplus C^{-2 i}\right) \quad \text { and } \quad C^{\text {odd }}=\bigoplus_{i \geqslant 0}\left(C^{2 i+1} \oplus C^{-2 i-1}\right)
$$

and we recall that $\operatorname{det}\left(C^{\bullet}\right)=\bigotimes \operatorname{det}\left(C^{i}\right)^{(-1)^{i}}$.

There is a natural map (given by reordering)

$$
v_{C} \bullet \operatorname{det}\left(C^{\bullet}\right) \rightarrow \operatorname{det}\left(C^{\text {ev }}\right) \otimes \operatorname{det}\left(C^{\text {odd }}\right)^{-1} .
$$

Remark 2.3. (a) If $D^{\bullet}$ is a further $K$-complex and if all the terms of $C^{\bullet}$ and $D^{\bullet}$ have even dimension, then the map

$$
\operatorname{det}\left(C^{\bullet} \oplus D^{\bullet}\right) \cong \operatorname{det}\left(C^{\bullet}\right) \otimes \operatorname{det}\left(D^{\bullet}\right)
$$

given by using the Koszul-twist isomorphisms coincides with the naive map given by the reordering of terms.

(b) If again all the terms $C^{i}$ have even dimension, then the map

$$
\operatorname{det}\left(C^{\bullet}\right) \rightarrow \operatorname{det}\left(C^{\mathrm{ev}}\right) \otimes \operatorname{det}\left(C^{\text {odd }}\right)^{-1}
$$

given by using the Koszul-twist isomorphisms coincides with the naive map $v_{C}$ e given by the reordering of terms.

We shall write $\mathrm{H}^{\bullet}\left(C^{\bullet}\right)$ for the complex $\left\{\mathrm{H}^{i}\left(C^{\bullet}\right)\right\}_{i}$, with zero boundary maps. As above, we write

$$
\mathrm{H}^{\mathrm{ev}}=\mathrm{H}^{\mathrm{ev}}\left(C^{\bullet}\right)=\mathrm{H}^{0}\left(C^{\bullet}\right) \underset{i>0}{\bigoplus}\left(\mathrm{H}^{2 i}\left(C^{\bullet}\right) \oplus \mathrm{H}^{-2 i}\left(C^{\bullet}\right)\right)
$$

and

$$
\mathrm{H}^{\text {odd }}=\mathrm{H}^{\text {odd }}\left(C^{\bullet}\right)=\bigoplus_{i \geqslant 0}\left(\mathrm{H}^{2 i+1}\left(C^{\bullet}\right) \oplus \mathrm{H}^{-2 i-1}\left(C^{\bullet}\right)\right) .
$$

From [KM76] we recall that there is a canonical isomorphism of $K$-lines

$$
\xi: \operatorname{det}\left(C^{\bullet}\right) \cong \operatorname{det}\left(\mathrm{H}^{\bullet}\left(C^{\bullet}\right)\right)
$$




\section{T. Chinburg, G. Pappas and M. Taylor}

Definition 2.4. Given alternating forms $h^{\mathrm{ev}}$ on $\mathrm{H}^{\mathrm{ev}}$ and $h^{\text {odd }}$ on $\mathrm{H}^{\text {odd }}$, define $\mathrm{Pf}_{h}$ to be the linear functional $\mathrm{Pf}_{h}: \operatorname{det}\left(\mathrm{H}^{\bullet}\left(C^{\bullet}\right)\right) \rightarrow K$ given by composing

$$
\mathrm{Pf}_{h^{\text {ev }}} \otimes \mathrm{Pf}_{h^{\text {odd }}}^{-1}: \operatorname{det}\left(\mathrm{H}^{\mathrm{ev}}\left(C^{\bullet}\right)\right) \otimes \operatorname{det}\left(\mathrm{H}^{\text {odd }}\left(C^{\bullet}\right)\right)^{-1} \rightarrow K
$$

with the isomorphism $v_{\mathrm{H}} \bullet(C \bullet)$. Note that in the sequel, for brevity, we shall usually write $h$ for the pair $\left\{h^{\mathrm{ev}}, h^{\text {odd }}\right\}$. Thus we now have defined the functional on $\operatorname{det}\left(C^{\bullet}\right)$ given by

$$
\mathrm{Pf}_{h} \circ \xi: \operatorname{det}\left(C^{\bullet}\right) \rightarrow K
$$

\subsection{Equivariant Pfaffians and the construction of sgn.pf}

Suppose now that $G$ is a finite group and that $K$ is a field of characteristic 0 . Let $W$ be a symplectic representation of $G$ defined over an extension $K^{\prime}$ of $K$. By definition, $W$ supports a non-degenerate $G$-invariant alternating form $\kappa: W \times W \rightarrow K^{\prime}$. Thus $\operatorname{dim}_{K^{\prime}}(W)$ is even.

A symmetric $K[G]$-space is a pair $(M, \sigma)$ where $M$ is a finitely generated $K[G]$-module which supports a non-degenerate $G$-invariant symmetric form $\sigma: M \times M \rightarrow K$. In this section, tensor products will be over $K$ unless otherwise specified. The $K^{\prime}$-space $(W \otimes M)^{G}$ supports the alternating non-degenerate form $(\kappa \otimes \sigma)^{G}$ which is the restriction of $\kappa \otimes \sigma$. So we have the Pfaffian

$$
\operatorname{Pf}_{(\kappa \otimes \sigma)^{G}}: \operatorname{det}\left((W \otimes M)^{G}\right) \rightarrow K^{\prime} .
$$

We will be concerned with the following basic construction.

Definition 2.5. Let $(U, \sigma)$ be a symmetric $K[G]$-space with $U$ a finitely generated free left $K[G]$ module. Fix a $K[G]$-isomorphism $f: K[G] \otimes L \rightarrow U$ in which $L$ is a finite-dimensional $K$-space having a trivial action of $G$. We have an isomorphism

$$
r_{W}: W \otimes L \rightarrow(W \otimes K[G] \otimes L)^{G}
$$

of $K^{\prime}$-vector spaces given by $r_{W}(w \otimes l)=\sum_{g} g w \otimes g \otimes l$ and an isomorphism

$$
f_{W}:(W \otimes K[G] \otimes L)^{G} \rightarrow(W \otimes U)^{G}
$$

induced by $f$. Denote by $\operatorname{Pf}(\kappa, f, \sigma)$ the composite map

$$
\begin{aligned}
K^{\prime} \otimes_{K} \operatorname{det}(L)^{\operatorname{dim}(W)} & \cong \operatorname{det}(W)^{\operatorname{dim}(L)} \otimes \operatorname{det}(L)^{\operatorname{dim}(W)} \\
& =\operatorname{det}(W \otimes L) \cong \operatorname{det}(W \otimes U)^{G} \rightarrow K^{\prime},
\end{aligned}
$$

where the first isomorphism is induced by the inverse of the isomorphism of lines $\operatorname{Pf}_{\kappa}: \operatorname{det}(W) \rightarrow K^{\prime}$, the second isomorphism is from (2.1), the third isomorphism results from $f_{W} \circ r_{W}$, and the final arrow is $\operatorname{Pf}_{(\kappa \otimes \sigma)^{G}}$.

In the next section we will prove the following result.

Proposition 2.6. Suppose that $K \subseteq \mathbb{R}$ and $K^{\prime} \subseteq \mathbb{C}$. For any $K$-basis $\left\{l_{i}\right\}$ of $L, \operatorname{Pf}(\kappa, f, \sigma)$ $\left(\bigwedge l_{i} \operatorname{dim}(W)\right)$ is a real number whose sign is independent of the choice of $\left\{l_{i}\right\}$, of $f$ and of the alternating form $\kappa$ on $W$. We denote this sign by $\operatorname{sgn} \cdot \operatorname{pf}(W, \sigma, U)$.

We now discuss two examples of Definition 2.5.

Example 2.7. Let $\nu$ denote the $K$-linear $G$-invariant symmetric form on $K[G]$ given by $\nu\left(g, g^{\prime}\right)=\delta_{g, g^{\prime}}$ for $g, g^{\prime} \in G$. Suppose $(L, s)$ is a symmetric $K$-space. Then

$$
r_{W}:(W \otimes L,|G| \cdot(\kappa \otimes s)) \rightarrow\left((W \otimes K[G] \otimes L)^{G},(\kappa \otimes \nu \otimes s)^{G}\right)
$$




\section{Pfaffians AND Hodge DiscRiminants}

is an isometry, since

$$
\begin{aligned}
(\kappa \otimes \nu \otimes s)^{G}\left(r_{W}(w \otimes l), r_{W}\left(w^{\prime} \otimes l^{\prime}\right)\right) & =(\kappa \otimes \nu \otimes s)\left(\sum_{g} g w \otimes g \otimes l, \sum_{g^{\prime}} g^{\prime} w^{\prime} \otimes g^{\prime} \otimes l^{\prime}\right) \\
& =\sum_{g, g^{\prime} \in G} \kappa\left(g w, g^{\prime} w^{\prime}\right) \cdot \nu\left(g, g^{\prime}\right) \cdot s\left(l, l^{\prime}\right) \\
& =\sum_{g=g^{\prime} \in G} \kappa\left(g w, g^{\prime} w^{\prime}\right) \cdot s\left(l, l^{\prime}\right) \\
& =|G| \cdot \kappa\left(w, w^{\prime}\right) \cdot s\left(l, l^{\prime}\right) \\
& =|G| \cdot(\kappa \otimes s)\left(w \otimes l, w^{\prime} \otimes l^{\prime}\right) .
\end{aligned}
$$

Suppose now that $f:(K[G] \otimes L, \nu \otimes s) \rightarrow(U, \sigma)$ is an isometry. Then

$$
|G|^{\operatorname{dim}_{K^{\prime}}(W \otimes L) / 2} \cdot \operatorname{Pf}_{\kappa \otimes s}=\operatorname{Pf}_{|G| \kappa \otimes s}=\operatorname{Pf}_{(\kappa \otimes \nu \otimes s)^{G}} \circ \operatorname{det}\left(r_{W}\right)=\operatorname{Pf}_{(\kappa \otimes \sigma)^{G}} \circ \operatorname{det}\left(f_{W} \circ r_{W}\right) .
$$

Example 2.8. Suppose $L=\bigoplus_{i=1}^{q} K$ in Example 2.7 is endowed with the quadratic form $s=\mathbf{1}^{(q)}$ given by $\sum_{i} x_{i}^{2}$. Let $U=\bigoplus_{i=1}^{q} K[G]=K[G] \otimes L$ be endowed with the form $\sigma=\nu^{(q)}=\nu \otimes \mathbf{1}^{(q)}$, which is the orthogonal sum of $q$ copies of $\nu$. The identity map $f:(K[G] \otimes L, \nu \otimes s) \rightarrow(U, \sigma)$ is an isometry. By (2.7) we have the equality

$$
|G|^{q n} \cdot \operatorname{Pf}_{\kappa \otimes \mathbf{1}^{(q)}}=\operatorname{Pf}_{\left(\kappa \otimes \nu^{(q)}\right)^{G}} \circ \operatorname{det}\left(f_{W} \circ r_{W}\right),
$$

where $f_{W}$ is the identity map and $2 n=\operatorname{dim}_{K^{\prime}}(W)$. Let $\left\{l_{i}\right\}$ be an orthonormal $K$-basis of $L$ with respect to $\mathbf{1}^{(q)}$, and let $\left\{v_{j}\right\}_{j=1}^{n} \cup\left\{v_{j}^{*}\right\}_{j=1}^{n}$ be a hyperbolic basis for $W$ over $K^{\prime}$, so that $\kappa\left(v_{i}, v_{j}^{*}\right)=\delta_{i, j}$ and $\kappa\left(v_{i}, v_{j}\right)=\kappa\left(v_{i}^{*}, v_{j}^{*}\right)=0$. Unwinding the definitions using these choices leads to

$$
\operatorname{Pf}(\kappa, f, \sigma)\left(\bigwedge l_{i}^{\operatorname{dim}(W)}\right)=|G|^{q n} .
$$

Changing the basis $\left\{l_{i}\right\}$ by an automorphism $\alpha$ of $L$ multiplies $\operatorname{Pf}(\kappa, f, \sigma)\left(\bigwedge l_{i}{ }^{\operatorname{dim}(W)}\right)$ by $\operatorname{det}(\alpha)^{2 n}$. Since $\operatorname{det}(\alpha) \in K^{*} \subset \mathbb{R}^{*}$, it follows that $\operatorname{Pf}(\kappa, f, \sigma)\left(\bigwedge l_{i}{ }^{\operatorname{dim}(W)}\right)$ is positive for an arbitrary $K$-basis $\left\{l_{i}\right\}$ of $L$. This proves Proposition 2.6 when $\sigma=\nu^{(q)}$ and shows that

$$
\operatorname{sgn} \cdot \operatorname{pf}\left(W, \nu^{(q)}, U\right)=\operatorname{sign}\left(\operatorname{Pf}\left(\kappa, f, \nu^{(q)}\right)\left(\bigwedge l_{i}^{\operatorname{dim}(W)}\right)\right)=1 .
$$

We end this section with an elementary but important result.

Lemma 2.9. Suppose that $K \subseteq \mathbb{R}$ and that $M$ is a finitely generated $K[G]$-module. Then $\operatorname{dim}\left(W \otimes_{K}\right.$ $M)^{G}$ is even.

Proof. It will suffice to treat the case $K=\mathbb{R}$. Then $M$ supports a positive definite $G$-invariant symmetric bilinear form. Hence $\left(W \otimes_{\mathbb{R}} M\right)^{G}$ supports a non-degenerate alternating form, so this space has even dimension over $K^{\prime}$.

\subsection{Evaluation of Pfaffians}

We assume in this section the notation of the previous section and that the field $K$ is a subfield of $\mathbb{R}$. Our object is to prove Proposition 2.6.

If $V$ is a left $A=K[G]$-module, we give $V$ the right $A$-module structure for which $v r=\bar{r} v$ if $r \in A$ and $v \in V$, where $r \rightarrow \bar{r}$ is the $K$-linear involution on $A$ sending $g \in G$ to $g^{-1}$. Suppose $U$ is a free left $A=K[G]$-module which supports a $K$-valued non-degenerate $G$-invariant symmetric form $\sigma$. We write $\widetilde{\sigma}: U \times U \rightarrow K[G]=A$ for the associated group ring-valued hermitian form 


\section{T. Chinburg, G. Pappas and M. Taylor}

(cf. [Frö84, p. 25]) defined by

$$
\widetilde{\sigma}\left(u, u^{\prime}\right)=\sum_{g \in G} \sigma\left(g u, u^{\prime}\right) g^{-1}
$$

for $u, u^{\prime} \in U$. An easy calculation shows that $\widetilde{\sigma}$ is $A$-linear in the first variable and hermitian, in the sense that $\tilde{\sigma}\left(u^{\prime}, u\right)=\overline{\widetilde{\sigma}\left(u, u^{\prime}\right)}$. Choose a basis $\left\{u_{i}\right\}_{i=1}^{q}$ for $U$ as a left $A$-module. Relative to this basis let $\nu^{(q)}: U \times U \rightarrow K$ be the symmetric bilinear form on $U$ which is the orthogonal sum of $q$ copies of the form $\nu: A \times A \rightarrow K$ defined by $\nu(g, h)=\delta_{g, h}$ for $g, h \in G$.

Since $\sigma$ is non-degenerate, we find that there is a unique $T \in \operatorname{Hom}_{A}(U, U)$ such that

$$
\widetilde{\sigma}\left(u^{\prime}, u\right)=\widetilde{\nu^{(q)}}\left(T\left(u^{\prime}\right), u\right) .
$$

Since $\widetilde{\sigma}$ and $\widetilde{\nu^{(q)}}$ are hermitian, we find that $T$ is self-adjoint with respect to $\widetilde{\nu^{(q)}}$.

We now adopt the notation of Example 2.8. Thus $\kappa$ is an alternating $G$-invariant $K^{\prime}$-valued form on the $K^{\prime}[G]$-module $W$, and $L=K^{q}=\bigoplus_{i=1}^{q} K l_{i}$ has the quadratic form $s=\mathbf{1}^{(q)}$ given by $\sum_{i} x_{i} l_{i} \rightarrow \sum_{i} x_{i}^{2}$. Write $A=K[G]$. Fix a basis $\left\{u_{i}\right\}_{i}$ for $U$ as a left $A$-module, and identify $U$ with $A \otimes_{K} L$ by sending $u_{i}$ to $1 \otimes l_{i}$. Recall that the left $K^{\prime}[G]$-module structure of $W$ gives a right $K^{\prime}[G]$-module structure via $w r=\bar{r} w$ for $r \in K^{\prime}[G]$ and $w \in W$. This identifies $W \otimes_{A} U$ with the direct sum $W^{q}=W \otimes_{A} A \otimes_{K} L=W \otimes_{K} L$ of $q$ copies of $W$. Let $\nu^{(q)}=\nu \otimes \mathbf{1}^{(q)}$ be the form on $U$ which is the orthogonal sum of $q$ copies of the symmetric non-degenerate $G$-invariant $K$-valued form $\nu$ on $A$. Let $\kappa^{(q)}=\kappa \otimes \mathbf{1}^{(q)}$ be the form on $W^{q}=W \otimes_{K} L$ which is the orthogonal direct sum of $\kappa$ on $q$ copies of $W$. Thus $\kappa^{(q)}$ and $\nu^{(q)}$ are $G$-invariant and non-degenerate.

The left $A$-linear map $T: U \rightarrow U$ induces the $K^{\prime}$-linear map

$$
T_{W}^{(q)}=1 \otimes_{A} T: W^{q}=W \otimes_{A} U \rightarrow W \otimes_{A} U=W^{q} .
$$

This map will not in general be $G$-equivariant with respect to the left action of $G$ on $W^{q}$, but this will not matter in the arguments below.

Proposition 2.10. Let $U, W$ and $\kappa$ be as above.

(a) The map $T_{W}^{(q)}$ is self-adjoint with respect to $\kappa^{(q)}$.

(b) Let $K^{\prime \prime}$ be the extension of $K$ generated by the value of the character $\chi_{W}$ of $W$. Then $\mathbf{p f}_{\kappa^{(q)}}\left(T_{W}^{(q)}\right)$ is a non-zero element of $K^{\prime \prime}$ which depends only on $\chi_{W}$ and not on either the particular representation $W$ or the choice of form $\kappa$. In particular, if $K=\mathbb{R}$ and $K^{\prime \prime}=\mathbb{C}$, then $\mathbf{p f}_{\kappa^{(q)}}\left(T_{W}^{(q)}\right)$ is a non-zero real number.

(c) One has

$$
\left.\operatorname{Pf}_{(\kappa \otimes \sigma)^{G}}=\mathbf{p f}_{\kappa^{(q)}}\left(T_{W}^{(q)}\right) \cdot \operatorname{Pf}_{(\kappa \otimes \nu(q)}\right)^{G} .
$$

Proof. Part (a) follows from the fact that $T$ is self-adjoint with respect to $\nu^{(q)}$. Part (b) is a consequence of this together with Propositions 4.2 and 4.3 in [Frö84, p. 37] and the fact that symplectic characters over $\mathbb{C}$ are real-valued.

To prove part (c), we evaluate the coefficient of the identity element of $G$ in (2.11) to have

$$
\sigma\left(u^{\prime}, u\right)=\nu^{(q)}\left(T\left(u^{\prime}\right), u\right)
$$

Therefore for $w, w^{\prime} \in W$ one has

$$
(\kappa \otimes \sigma)\left(w^{\prime} \otimes u^{\prime}, w \otimes u\right)=\left(\kappa \otimes \nu^{(q)}\right)\left((1 \otimes T)\left(w^{\prime} \otimes u^{\prime}\right), w \otimes u\right) .
$$

This implies that

$$
(\kappa \otimes \sigma)^{G}\left(m^{\prime}, m\right)=\left(\kappa \otimes \nu^{(q)}\right)\left((1 \otimes T)^{G}\left(m^{\prime}\right), m\right)
$$




\section{PfaffiAns AND HodGe DISCRIMINANTS}

for $m^{\prime}, m \in(W \otimes U)^{G}$. Hence Proposition 2.1 shows that

$$
\operatorname{Pf}_{(\kappa \otimes \sigma)^{G}}=\mathbf{p f}_{\left(\kappa \otimes \nu^{(q)}\right)^{G}}\left((1 \otimes T)^{G}\right) \cdot \operatorname{Pf}_{\left(\kappa \otimes \nu^{(q)}\right)^{G}} .
$$

In Example 2.7 we now let $(L, s)$ be $\left(K^{q}, 1_{q}^{(q)}\right)$, and we let $f:(K[G] \otimes L, \nu \otimes s) \rightarrow\left(U, \nu^{(q)}\right)$ be the canonical isometry. We conclude from (2.7) that

$$
\operatorname{Pf}_{|G| \kappa(q)}=\operatorname{Pf}_{|G| \kappa \otimes s}=\operatorname{Pf}_{\left(\kappa \otimes \nu^{(q)}\right)^{G}} \circ \operatorname{det}\left(f_{W} \circ r_{W}\right),
$$

where $r_{W}$ is defined in (2.5), and both sides of (2.17) are linear functionals on $\operatorname{det}\left(W \otimes_{K} L\right)=$ $\operatorname{det}\left(W^{(q)}\right)$. From the definition of $T_{W}^{(q)}$ there is the following commutative diagram.

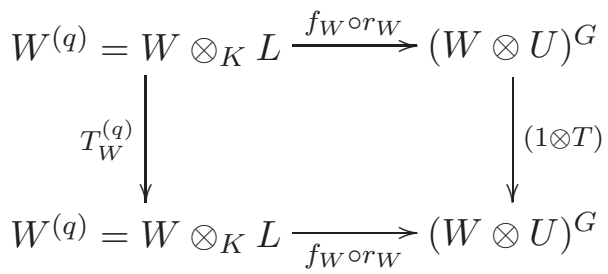

As in Example 2.7, $f_{W} \circ r_{W}$ is an isometry when $W^{(q)}$ (respectively $(W \otimes U)^{G}$ ) is given the form $|G| \kappa^{(q)}$ (respectively $\left(\kappa \otimes \nu^{(q)}\right)^{G}$ ). We now conclude from $(2.17)$, (2.18) and the definition of Pfaffians of automorphisms in Proposition 2.1 that

$$
\mathbf{p f}_{\left(\kappa \otimes \nu^{(q)}\right)^{G}}\left((1 \otimes T)^{G}\right)=\mathbf{p f}_{|G| \kappa^{(q)}}\left(T_{W}^{(q)}\right)=\mathbf{p f}_{\kappa^{(q)}}\left(T_{W}^{(q)}\right),
$$

where the second equality is a consequence of Remark 2.2. Combining (2.16) and (2.19) gives the equality (2.12) of part (c).

Proof of Proposition 2.6. Suppose $K \subseteq \mathbb{R}$. We are to show that for any $K$-basis $\left\{l_{i}\right\}$ of $L, \operatorname{Pf}(\kappa, f, \sigma)$ $\left(\bigwedge l_{i}{ }^{\operatorname{dim}(W)}\right)$ is a real number whose sign is independent of the choice of $\left\{l_{i}\right\}$, of $f$ and of the alternating form $\kappa$ on $W$.

By Proposition 2.10(c) and Definition 2.5 we have

$$
\operatorname{Pf}(\kappa, f, \sigma)\left(\bigwedge l_{i}^{\operatorname{dim}(W)}\right)=\mathbf{p f}_{\kappa^{(q)}}\left(T_{W}^{(q)}\right) \cdot \operatorname{Pf}\left(\kappa, f, \nu^{(q)}\right)\left(\bigwedge l_{i}^{\operatorname{dim}(W)}\right) .
$$

By Proposition 2.10(b), the constant $\mathbf{p f}_{\kappa^{(q)}}\left(T_{W}^{(q)}\right)$ depends only on the character $\chi_{W}$ of $W$. By Example 2.8, the sign of $\operatorname{Pf}\left(\kappa, f, \nu^{(q)}\right)\left(\bigwedge l_{i}{ }^{\operatorname{dim}(W)}\right)$ is positive, independent of the choice of $\left\{l_{i}\right\}, f$ and $\kappa$. So Proposition 2.6 follows from $(2.20)$.

We note the following corollary of the proof.

Corollary 2.11. One has

$$
\operatorname{sgn} \cdot \operatorname{pf}(W, \sigma, U)=\operatorname{sign}\left(\operatorname{Pf}(\kappa, f, \sigma)\left(\bigwedge l_{i}^{\operatorname{dim}(W)}\right)\right)=\operatorname{sign}\left(\mathbf{p f}_{\kappa^{(q)}}\left(T_{W}^{(q)}\right)\right) .
$$

\subsection{Pfaffians and signatures}

In this section we let the field $K$ be $\mathbb{R}$. We shall determine the signs of Pfaffians in terms of signature invariants. We first need the following algebraic result.

Proposition 2.12. Given an $\mathbb{R}[G]$-module $M$ and a non-degenerate $G$-invariant symmetric form $\sigma: M \times M \rightarrow \mathbb{R}$ on $M$, there exists a $G$-decomposition $M=M^{+} \oplus M^{-}$where $\sigma$ is positive definite on $M^{+}$and negative definite on $M^{-}$. This decomposition is not necessarily unique, but the characters of the action of $G$ on $M^{+}$and $M^{-}$are independent of choices. 


\section{T. Chinburg, G. Pappas and M. Taylor}

Proof. For full details see [AS68, p. 578]; we briefly sketch a proof for the reader's convenience. First we choose a $G$-invariant positive definite symmetric form $\tau$ on $M$; there is then a unique automorphism $A$ of $M$ such that, for all $x, y \in M$,

$$
\sigma(x, y)=\tau(x, A y) .
$$

As both $\sigma$ and $\tau$ are symmetric, $A$ is self-adjoint with respect to $\tau$; furthermore, since both $\sigma$ and $\tau$ are $G$-invariant, $A$ commutes with the action of $G$. Thus the different eigenspaces of $A$ are preserved by $G$, so by considering the sums of eigenspaces for positive and negative eigenvalues, we obtain the required decomposition $M=M^{+} \oplus M^{-}$.

Clearly the above decomposition depends on the choice of $\tau$. To see that the characters of $M^{+}$ and $M^{-}$are independent of the choice of $\tau$, we note that: the space of positive definite $G$-invariant forms on $M$ is connected; the maps $\tau \mapsto \operatorname{char}\left(M^{ \pm}\right)$are continuous; and $\operatorname{char}\left(M^{ \pm}\right)$takes values in the discrete group $R_{G}$.

A particularly simple, but nonetheless useful, instance of the above decomposition occurs when $(M, \sigma)$ is hyperbolic. To state this result we first need some notation. Recall that for an $\mathbb{R}[G]$-module $V$ the hyperbolic space $\operatorname{Hyp}(V)=V \oplus V^{D}$ is endowed with the form $h$,

$$
h\left(v \oplus f, v^{\prime} \oplus f^{\prime}\right)=f\left(v^{\prime}\right)+f^{\prime}(v) \quad \text { for } v, v^{\prime} \in V, f, f^{\prime} \in V^{D} .
$$

Lemma 2.13. There are $\mathbb{R}[G]$-isomorphisms $\operatorname{Hyp}(V)^{+} \cong V \cong \operatorname{Hyp}(V)^{-}$.

Proof. We can reduce to the case in which $V$ is a simple $\mathbb{R}[G]$-module. The lemma then follows from the fact that $V$ is isomorphic to $V^{D}$ as an $\mathbb{R}[G]$-module since $V$ has real character, and $\operatorname{dim}\left(\operatorname{Hyp}(V)^{+}\right)=\operatorname{dim}\left(\operatorname{Hyp}(V)^{-}\right)$since $\sigma=h$ has signature 0.

Proposition 2.14. Let $U$ be a free $\mathbb{R}[G]$-module with basis $\left\{u_{i}\right\}, i=1, \ldots, q$, and suppose that $U$ supports a non-degenerate real-valued $G$-invariant form $\sigma$. Choose a decomposition $U=U^{+} \oplus U^{-}$, as in Proposition 2.12, and define $n_{W}^{ \pm}(\sigma)=\operatorname{dim}\left(W \otimes U^{ \pm}\right)^{G}$. Then

$$
\operatorname{sgn} \cdot \operatorname{pf}(W, \sigma, U)=(\sqrt{-1})^{n_{W}^{-}(\sigma)} .
$$

Note that by Lemma 2.9 the integers $n_{W}^{ \pm}(\sigma)$ are all even, since they are the multiplicities of symplectic representations in real representations.

Proof. Recall that the form $\nu$ on $\mathbb{R}[G]$ is defined by $\nu\left(g, g^{\prime}\right)=\delta_{g, g^{\prime}}$ for $g, g^{\prime} \in G$. The direct $\operatorname{sum} \nu^{(q)}$ of $q$ copies of $\nu$ gives a $G$-invariant positive definite form on $U$. As in the proof of Proposition 2.12, there is a unique $\mathbb{R}[G]$-automorphism $A$ of $U$, which is self-adjoint with respect to $\nu$, such that, for all $x, y \in U, \sigma(x, y)=\nu^{(q)}(x, A y)$. Therefore the decomposition $U=U^{+} \oplus U^{-}$induces a decomposition

$$
(W \otimes U)^{G}=\left(W \otimes U^{+}\right)^{G} \oplus\left(W \otimes U^{-}\right)^{G}
$$

and the restriction $(1 \otimes A)^{G}$ of $1 \otimes A$ to $(W \otimes U)^{G}$ is diagonalizable on the subspaces $\left(W \otimes U^{ \pm}\right)^{G}$ with eigenvalues of sign \pm 1 . By Proposition 2.1

$$
\mathrm{Pf}_{(\kappa \otimes \sigma)^{G}}=\mathbf{p f}_{\left(\kappa \otimes \nu^{(q)}\right)^{G}}\left((1 \otimes A)^{G}\right) \cdot \operatorname{Pf}_{\left(\kappa \otimes \nu^{(q)}\right)^{G}} .
$$

Comparing with Proposition 2.10(c), we deduce that

$$
\mathbf{p f}_{\left(\kappa \otimes \nu^{(q)}\right)^{G}}\left((1 \otimes A)^{G}\right)=\mathbf{p f}_{\kappa^{(q)}}\left(T_{W}^{(q)}\right)
$$

and the sign of the latter term is $\operatorname{sgn} \cdot \operatorname{pf}(W, \sigma, U)$ by Corollary 2.11. The result then follows by repeated use of the fact that (see [Frö84, p. 40])

$$
\text { pf }\left(\begin{array}{ll}
d & 0 \\
0 & d
\end{array}\right)=d
$$




\section{Pfaffians AND Hodge Discriminants}

For $i=0,1$, let $\left(P_{i}, \sigma_{i}\right)$ and $\left(V_{i}, \tau_{i}\right)$ be symmetric $\mathbb{R}[G]$-spaces which become isometric over $\mathbb{C}$, so that there is a $\mathbb{C}[G]$-module isomorphism $c_{i}: V_{i} \otimes \mathbb{C} \cong P_{i} \otimes \mathbb{C}$ which carries the $\mathbb{C}$-valued form $\tau_{i} \otimes 1$ to $\sigma_{i} \otimes 1$. Let $\langle\iota\rangle=\operatorname{Gal}(\mathbb{C} / \mathbb{R})$, and let $\iota$ act on $P_{i} \otimes \mathbb{C}$ via the second factor. Suppose that, under $c_{i}, V_{i}$ is identified with an $\mathbb{R}[G]$-submodule of $P_{i} \otimes \mathbb{C}$ which is stable under the action of $\iota$. Recall that $P_{i}=P_{i}^{+} \oplus P_{i}^{-}$where $P_{i}^{+}$(respectively $P^{-}$) is an $\mathbb{R}[G]$-submodule of $P_{i}$ on which $\sigma_{i}$ is positive (respectively negative) definite. We have a similar decomposition $V_{i}=V_{i}^{+} \oplus V_{i}^{-}$of $V_{i}$ with respect to $\tau_{i}$. If $M$ is an $\mathbb{R}[G]$-submodule of $P_{i} \otimes \mathbb{C}$ for $i=0$ or $i=1$, let $M_{+}$(respectively $M_{-}$) be the subspace on which $\iota$ acts by multiplication by 1 (respectively -1 ). Since the actions of $G$ and of $\iota$ commute, $M_{+}$and $M_{-}$are $\mathbb{R}[G]$-submodules. Define $M_{W}=\left(W \otimes_{\mathbb{R}} M\right)^{G}$. We will abbreviate $\left(P_{i}^{ \pm}\right)_{W}$ by $P_{i, W}^{ \pm},\left(\left(V_{i}^{ \pm}\right)_{+}\right)_{W}$ by $V_{i,+, W}^{ \pm}$and $\left(\left(V_{i}^{ \pm}\right)_{-}\right)_{W}$ by $V_{i,-, W}^{ \pm}$.

Proposition 2.15. Let $P$ (respectively $V$ ) denote the virtual $\mathbb{R}[G]$-module $P_{0}-P_{1}$ (respectively $\left.V_{0}-V_{1}\right)$.

(a) One has

and

$$
\operatorname{dim}\left(P_{i, W}^{+}\right)=\operatorname{dim}\left(V_{i,+, W}^{+}\right)+\operatorname{dim}\left(V_{i,-, W}^{-}\right)
$$

$$
\operatorname{dim}\left(P_{i, W}^{-}\right)=\operatorname{dim}\left(V_{i,+, W}^{-}\right)+\operatorname{dim}\left(V_{i,-, W}^{+}\right) .
$$

(b) Suppose that $V^{+}=V_{0}^{+}-V_{1}^{+}$and $V^{-}=V_{0}^{-}-V_{1}^{-}$are free virtual $\mathbb{R}[G]$-modules. If $W$ is a virtual symplectic representation of $G$ of dimension 0, then $\operatorname{dim}\left(P_{W}^{-}\right) \equiv \operatorname{dim}\left(V_{-, W}\right) \bmod 4$. Furthermore, if each of the $\left(P_{i}, \sigma_{i}\right)$ is hyperbolic, then $\operatorname{dim}\left(P_{W}^{-}\right)=0$.

(c) Let $T$ denote the trivial representation of $G$ and suppose that $W$ is the symplectic representation afforded by two copies of $T$. Then $\operatorname{dim}\left(P_{W}^{-}\right) \equiv 2 \operatorname{dim}\left(V_{T}^{-}\right)+2 \operatorname{dim}\left(V_{-, T}\right) \bmod 4$. Furthermore, if $\left(P_{i}, \sigma_{i}\right)$ is hyperbolic for $i=1,2$, then $\operatorname{dim}\left(P_{W}^{-}\right)=\operatorname{dim}\left(V_{T}\right)$.

Proof. To prove part (a), let $\sigma_{i}^{\prime}$ (respectively $\tau_{i}^{\prime}$ ) be the real part of the $\mathbb{C}$-valued form $\sigma_{i} \otimes 1$ (respectively $\tau_{i} \otimes 1$ ) on $P_{i} \otimes \mathbb{C}$ (respectively $V_{i} \otimes \mathbb{C}$ ). Via $c_{i}$ we consider $V_{i}$ as an $\mathbb{R}[G]$-submodule of $P_{i} \otimes \mathbb{C}$, and in this way $\sigma_{i}^{\prime}$ is identified with $\tau_{i}^{\prime}$. Since $\tau_{i}$ is real-valued on $V_{i}$, the spaces $V_{i,+}^{+}$ and $\sqrt{-1} V_{i,-}^{-}$are orthogonal with respect to $\tau_{i}^{\prime}=\sigma_{i}^{\prime}$, have trivial intersection, and are contained in $P_{i}=\left(P_{i} \otimes \mathbb{C}\right)_{+}$. Thus

$$
V_{i,+}^{+} \oplus \sqrt{-1} V_{i,-}^{-} \subset P_{i}^{+} \quad \text { and similarly } \quad V_{i,+}^{-} \oplus \sqrt{-1} V_{i,-}^{+} \subset P_{i}^{-} .
$$

Since

$$
\operatorname{dim}\left(P_{i}^{+}\right)+\operatorname{dim}\left(P_{i}^{-}\right)=\operatorname{dim}\left(P_{i}\right)=\operatorname{dim}\left(V_{i}\right)=\operatorname{dim}\left(V_{i,+}^{+}\right)+\operatorname{dim}\left(V_{i,-}^{-}\right)+\operatorname{dim}\left(V_{i,+}^{-}\right)+\operatorname{dim}\left(V_{i,-}^{-}\right),
$$

the inclusions in (2.22) must be equalities. Part (a) is now clear from the fact that $\sqrt{-1} V_{i,-}^{ \pm}$is isomorphic as an $\mathbb{R}[G]$-module to $V_{i,-}^{ \pm}$.

To prove part (b), first note that, since both $V^{ \pm}$are (virtual) free $G$-modules, then so is $V$. Hence $P$ is also a (virtual) free $G$-module. Next note from part (a) that

$$
\operatorname{dim}\left(P_{W}^{-}\right)=\operatorname{dim}\left(V_{+, W}^{-}\right)+\operatorname{dim}\left(V_{-, W}^{+}\right) .
$$

Then, since $W$ has dimension 0 and $V^{-}$is $\mathbb{R}[G]$-free, it follows that $\operatorname{dim}\left(V_{W}^{-}\right)=0$ and so $\operatorname{dim} V_{+, W}^{-}=$ $-\operatorname{dim} V_{-, W}^{-}$; hence, since all terms are even by Lemma 2.9, we have established the congruence

$$
\operatorname{dim}\left(P_{W}^{-}\right)=-\operatorname{dim}\left(V_{-, W}^{-}\right)+\operatorname{dim}\left(V_{-, W}^{+}\right) \equiv+\operatorname{dim}\left(V_{-, W}^{-}\right)+\operatorname{dim}\left(V_{-, W}^{+}\right) \bmod 4
$$

and the last expression is equal to $\operatorname{dim}\left(V_{-, W}\right)$. Finally note that if $P$ is $\mathbb{R}[G]$-free and if $\left(P_{i}, \sigma_{i}\right)$ is hyperbolic for $i=1,2$, then $\operatorname{dim}\left(P_{W}^{-}\right)=\operatorname{dim}\left(P_{W}\right) / 2=0$. 


\section{T. Chinburg, G. Pappas and M. Taylor}

To prove part (c) note that

$$
\begin{aligned}
\operatorname{dim}\left(P_{W}^{-}\right) & =\operatorname{dim}\left(V_{+, W}^{-}\right)+\operatorname{dim}\left(V_{-, W}^{+}\right)=2 \operatorname{dim}\left(V_{+, T}^{-}\right)+2 \operatorname{dim}\left(V_{-, T}^{+}\right) \\
& =2 \operatorname{dim}\left(V_{T}^{-}\right)-2 \operatorname{dim}\left(V_{-, T}^{-}\right)+2 \operatorname{dim}\left(V_{-, T}^{+}\right) \equiv 2 \operatorname{dim}\left(V_{T}^{-}\right)+2 \operatorname{dim}\left(V_{-, T}\right) \bmod 4 .
\end{aligned}
$$

If $\left(P_{i}, \sigma_{i}\right)$ is hyperbolic for $i=1,2$, then $\operatorname{dim}\left(P_{W}^{-}\right)=\operatorname{dim}\left(P_{W}\right) / 2=\operatorname{dim}\left(P_{T}\right)=\operatorname{dim}\left(V_{T}\right)$.

Finally, we state and prove the following result on filtered quadratic modules which we will need in calculating the signature invariants of Hodge cohomology in $\S 3$.

Lemma 2.16. Let $K$ be an arbitrary field of characteristic 0 , and let $\sigma$ be a non-degenerate $K$-valued $G$-invariant symmetric form on a finite-dimensional $K[G]$-module $V$. Suppose that $W$ is an isotropic $K[G]$-submodule of $V$ and let $W^{\perp}$ denote the space of vectors orthogonal to $W$. Then there is an orthogonal decomposition of $K[G]$-modules

$$
V \cong \operatorname{Hyp}(W) \oplus \frac{W^{\perp}}{W} .
$$

Suppose further that $(V, \sigma)$ is a filtered quadratic $K[G]$-space in the following sense. We are given an increasing filtration $\left\{F_{i}\right\}$ of $K[G]$-submodules with $F_{-N}=(0)$ and $F_{N}=V$ for $N \gg 0$, and with $F_{i}^{\perp}=F_{-i-1}$. Then for all $i, \sigma$ induces isomorphisms

$$
\mathrm{Gr}_{-i} \cong \mathrm{Gr}_{i}^{D},
$$

where $\mathrm{Gr}_{i}$ denotes the $i$ th graded piece $F_{i} / F_{i-1}$. There is a (non-canonical) $K[G]$-decomposition of quadratic modules

$$
V \cong \bigoplus_{i<0} \operatorname{Hyp}\left(\mathrm{Gr}_{i}\right) \oplus \mathrm{Gr}_{0}
$$

Proof. First choose an arbitrary decomposition of $K[G]$-modules $W^{\perp}=W \oplus U$. This is trivially an orthogonal decomposition, and because $\sigma$ is non-degenerate it has no kernel on $U=W^{\perp} / W$. Thus the restriction of $\sigma$ to $U$ is non-degenerate. We now choose an arbitrary further decomposition $U^{\perp}=W \oplus W^{\prime}$. Then the form $\sigma$ induces isomorphisms $W^{\prime} \rightarrow W^{D}$ and $W \rightarrow W^{\prime D}$. It follows that $W \oplus W^{\prime}$ is isomorphic to $\operatorname{Hyp}(W)$ so $V=W \oplus W^{\prime} \oplus U$ is a decomposition of the form in (2.23). The second part of the lemma then follows at once from the first part.

\subsection{Complexes of $K[G]$-modules}

Definition 2.17. A symmetric $K[G]$-complex is a pair $\left(C^{\bullet}, \sigma\right)$ where $C^{\bullet}$ is a perfect $K[G]$-complex and where $\sigma^{\text {ev }}$ and $\sigma^{\text {odd }}$ are non-degenerate real-valued $G$-invariant symmetric forms on $\mathrm{H}^{\mathrm{ev}}\left(C^{\bullet}\right)$ and $\mathrm{H}^{\text {odd }}\left(C^{\bullet}\right)$ respectively.

For a given symmetric complex $\left(C^{\bullet}, \sigma\right)$ and for $W$ and $\kappa$ as above, we $\operatorname{define} \operatorname{det}\left(C_{W}^{\bullet}\right)$ to be the line $\operatorname{det}\left(\left(W \otimes_{K} C^{\bullet}\right)^{G}\right)$; thus we have the canonical isomorphism

$$
\xi_{W}: \operatorname{det}\left(C_{W}^{\bullet}\right) \cong \operatorname{det}\left(\mathrm{H}^{\bullet}\left(C^{\bullet}\right)_{W}\right) .
$$

By restricting $\kappa \otimes \sigma^{\mathrm{ev}}$ to $\left(W \otimes \mathrm{H}^{\mathrm{ev}}\right)^{G}$ we obtain a non-degenerate alternating form which we denote by $\left(\kappa \otimes \sigma^{\text {ev }}\right)^{G}$; similarly we obtain a form $\left(\kappa \otimes \sigma^{\text {odd }}\right)^{G}$ on $\left(W \otimes \mathrm{H}^{\text {odd }}\right)^{G}$. Thus we obtain the composite map $\operatorname{det}\left(C_{W}^{\bullet} \cong \operatorname{det}\left(\mathrm{H}^{\bullet}\left(C^{\bullet}\right)_{W}\right) \rightarrow K\right.$ where the right-hand arrow is $\operatorname{Pf}_{(\kappa \otimes \sigma)^{G}}$, as constructed in Definition 2.4. In the sequel for brevity we shall henceforth often write $\operatorname{Pf}_{(\kappa \otimes \sigma)^{G}}$ in place of $\mathrm{Pf}_{(\kappa \otimes \sigma)^{G}} \circ \xi$. 


\section{Pfaffians AND Hodge DiscRiminants}

\section{Hodge, de Rham and Betti cohomology}

Throughout this section we adopt the following notation: $K$ again denotes a subfield of $\mathbb{R}$ and we suppose that the scheme $X$ is smooth, equidimensional of dimension $d$, and projective over $\operatorname{Spec}(K)$. We assume that $G$ acts freely on $X$, so that $\pi: X \rightarrow Y$ is a étale $G$-cover. We start by considering forms on the Hodge, de Rham and Betti cohomology of $X$ arising from Serre duality. We then consider in detail the behavior of signatures of these forms under the comparison isomorphism between de Rham and Betti cohomology.

\subsection{Pairings on Hodge cohomology}

We first recall some conventions regarding the tensor product of complexes $I^{\bullet}$ and $J^{\bullet}$ of objects in an abelian category. The total complex $\operatorname{Tot}\left(I^{\bullet} \otimes J^{\bullet}\right)$ of the bicomplex $I^{\bullet} \otimes J^{\bullet}$ has $n$th term

$$
\operatorname{Tot}\left(I^{\bullet} \otimes J^{\bullet}\right)=\bigoplus_{i+j=n} I^{i} \otimes J^{j}
$$

and differential $d$ which on the summand $I^{i} \otimes J^{j}$ on the right in (3.1) is

$$
\left(d_{I}^{i} \bullet \text { identity }\right)+(-1)^{i}\left(\text { identity } \otimes d_{J}^{j}\right) .
$$

It follows that there is an isomorphism of complexes

$$
\lambda: \operatorname{Tot}\left(I^{\bullet} \otimes J^{\bullet}\right) \rightarrow \operatorname{Tot}\left(J^{\bullet} \otimes I^{\bullet}\right)
$$

which sends $\alpha \otimes \beta \in I^{i} \otimes J^{j}$ to $(-1)^{i j}(\beta \otimes \alpha)$.

Suppose now that $\mathcal{F}$ and $\mathcal{G}$ are coherent sheaves on $X$, with injective resolutions $\mathcal{F} \rightarrow I^{\bullet}$ and $\mathcal{G} \rightarrow J^{\bullet}$. We then have acyclic resolutions $\mathcal{F} \otimes \mathcal{G} \rightarrow \operatorname{Tot}\left(I^{\bullet} \otimes J^{\bullet}\right)$ and $\mathcal{G} \otimes \mathcal{F} \rightarrow \operatorname{Tot}\left(J^{\bullet} \otimes I^{\bullet}\right)$. The isomorphism $\lambda$ above is compatible with the naive 'flip' isomorphism $\mathcal{F} \otimes \mathcal{G} \rightarrow \mathcal{G} \otimes \mathcal{F}$. It follows that when we use $\lambda$ to identify $\mathrm{H}^{i+j}(X, \mathcal{F} \otimes \mathcal{G})$ with $\mathrm{H}^{i+j}(X, \mathcal{G} \otimes \mathcal{F})$ we have

$$
x \cup y=(-1)^{i j} y \cup x
$$

for $x \in \mathrm{H}^{i}(X, \mathcal{F})$ and $y \in \mathrm{H}^{j}(X, \mathcal{G})$.

We now consider the Hodge cohomology group $\mathrm{H}^{i}\left(X, \Omega^{j}\right)$. Since $\Omega^{j}[-j]$ is a complex having $\Omega^{j}$ in degree $j$ and all other terms equal to 0 , we may identify $\mathrm{H}^{i}\left(X, \Omega^{j}\right)$ with $\mathrm{H}^{i+j}\left(X, \Omega^{j}[-j]\right)$ with no change of sign involving $i$ or $j$. The cup-product now gives $G$-equivariant duality pairings

$$
\sigma_{i, j}: \mathrm{H}^{i+j}\left(X, \Omega^{j}[-j]\right) \times \mathrm{H}^{2 d-i-j}\left(X, \Omega^{d-j}[-(d-j)]\right) \stackrel{\cup}{\rightarrow} \mathrm{H}^{2 d}\left(X, \Omega^{d}[-d]\right)=\mathrm{H}^{d}\left(X, \Omega^{d}\right) \stackrel{|G|^{-1} T r}{\longrightarrow} K,
$$

where $\operatorname{Tr}$ is the trace map; compare [Har77, ch. $7, \S \mathrm{III}]$. Note that here we divide the pairings used in [CPT03] by the group order. We used this normalization in [CPT02], and the reason for choosing this normalization will be explained in $\S 6.2$ (see also (3.16) below).

The isomorphism

$$
\operatorname{Tot}\left(\Omega^{j}[-j] \otimes \Omega^{d-j}[-(d-j)]\right) \rightarrow \operatorname{Tot}\left(\Omega^{d-j}[-(d-j)] \otimes \Omega^{j}[-j]\right)
$$

defined in (3.3) sends $\alpha \otimes \beta$ to $(-1)^{j(d-j)} \beta \otimes \alpha$ for $\alpha \in\left(\Omega^{j}[-j]\right)^{j}=\Omega^{j}$ and $\beta \in\left(\Omega^{d-j}[-(d-j)]\right)^{d-j}=$ $\Omega^{d-j}$. Here $\alpha \wedge \beta=(-1)^{j(d-j)} \beta \wedge \alpha$ in $\left(\Omega^{d}[-d]\right)^{d}=\Omega^{d}$. It follows that (3.6) is compatible with taking wedge products of forms. Therefore by the same reasoning showing (3.4) we have

$$
\sigma_{i, j}(x, y)=(-1)^{(i+j)(2 d-(i+j))} \sigma_{d-i, d-j}(y, x)=(-1)^{i+j} \sigma_{d-i, d-j}(y, x) .
$$

We then symmetrize these pairings by the construction given in [CPT03, $\S 3]$ : namely, we define the twisted pairing $\sigma_{i, j}^{\prime}$ by

$$
\sigma_{d-i, d-j}^{\prime}(y, x)=\sigma_{i, j}(x, y)=(-1)^{i+j} \sigma_{d-i, d-j}(y, x) .
$$




\section{T. Chinburg, G. Pappas and M. Taylor}

This gives us the following result.

Proposition 3.1. Suppose either that $d$ is odd or that if $d$ is even then at least one of $i$ and $j$ is different from $d / 2$. Then there is a $K[G]$-isometry

$$
\left(\mathrm{H}^{i+j}\left(X, \Omega^{j}[-j]\right) \oplus \mathrm{H}^{2 d-i-j}\left(X, \Omega^{d-j}[-(d-j)]\right), \sigma_{i, j} \oplus \sigma_{d-i, d-j}^{\prime}\right)=\operatorname{Hyp}\left(\mathrm{H}^{i+j}\left(X, \Omega^{j}[-j]\right)\right) .
$$

We follow the terminology of Grothendieck (see [Gro66]). For a given integer $t$, we consider the (shifted) $t$ th Hodge cohomology group

$$
\mathrm{H}_{\mathrm{Hod}}^{t}(X)[d]=\mathrm{H}^{t}\left(X, \bigoplus_{n} \Omega^{n}[d-n]\right)=\bigoplus_{n} \mathrm{H}^{t+d}\left(X, \Omega^{n}[-n]\right)
$$

and similarly we put

$$
\mathrm{H}_{\mathrm{Hod}}^{\mathrm{ev}}(X)[d]=\bigoplus_{t \text { even }} \mathrm{H}_{\mathrm{Hod}}^{t}(X)[d] \quad \text { and } \quad \mathrm{H}_{\mathrm{Hod}}^{\mathrm{odd}}(X)[d]=\bigoplus_{t \text { odd }} \mathrm{H}_{\mathrm{Hod}}^{t}(X)[d] .
$$

We now define pairings

$$
\sigma^{t}: \mathrm{H}_{\mathrm{Hod}}^{t}(X)[d] \times \mathrm{H}_{\mathrm{Hod}}^{-t}(X)[d] \rightarrow K
$$

as follows:

for $t<0$ we put

$$
\sigma^{t}=\bigoplus_{i+j=t+d} \sigma_{i, j}
$$

for $t>0$ we put

$$
\sigma^{t}=\bigoplus_{i+j=t+d} \sigma_{i, j}^{\prime}
$$

and for $t=0$ we set

$$
\sigma^{0}=\bigoplus_{i<d / 2} \sigma_{i, d-i} \oplus \sigma_{d / 2, d / 2} \bigoplus_{i>d / 2} \sigma_{i, d-i}^{\prime} .
$$

Here it is to be understood that the term $\sigma_{d / 2, d / 2}$ occurs only when $d$ is even. We note that in all cases,

$$
\sigma^{t}(x, y)=\sigma^{-t}(y, x)
$$

by (3.7) and (3.8). We now define the symmetric pairings

$$
\sigma^{\mathrm{ev}}=\bigoplus_{t \text { even }} \sigma^{t}, \quad \sigma^{\text {odd }}=\bigoplus_{t \text { odd }} \sigma^{t} .
$$

We then let $\left(\mathrm{H}_{\mathrm{Hod}}^{\mathrm{ev}}(X)[d], \sigma^{\mathrm{ev}}\right)$ denote $\mathrm{H}_{\mathrm{Hod}}^{\mathrm{ev}}(X)[d]$ endowed with the $G$-invariant symmetric form $\sigma^{\text {ev }}$ and similarly we have $\left(\mathrm{H}_{\mathrm{Hod}}^{\text {odd }}(X)[d], \sigma^{\text {odd }}\right)$. Note that Proposition 3.1 implies that $\sigma^{\text {odd }}$ is a hyperbolic pairing and that $\sigma^{\mathrm{ev}}$ is hyperbolic whenever $d$ is odd.

Since the signature of a hyperbolic form is always zero, we have the following result.

Lemma 3.2. For any symplectic representation $W$ of $G$

$$
n_{W}^{+}(\sigma)-n_{W}^{-}(\sigma)=n_{W}^{+}\left(\sigma_{d / 2, d / 2}\right)-n_{W}^{-}\left(\sigma_{d / 2, d / 2}\right),
$$

where the right-hand side is to be interpreted as zero if $d$ is odd.

\subsection{De Rham cohomology}

In this section we take $K=\mathbb{R}$ and we suppose that $d=\operatorname{dim}(X)$ is even, and we keep the notation of the previous paragraph. Applying Proposition 2.12 we have a decomposition of $\mathbb{R}[G]$-modules into 


\section{Pfaffians AND Hodge DiscRiminants}

positive and negative spaces,

$$
\mathrm{H}_{\mathrm{Hod}}^{\mathrm{ev}}(X)[d]_{\mathbb{R}}=\mathrm{H}_{\mathrm{Hod}}^{\mathrm{ev},+}[d] \oplus \mathrm{H}_{\mathrm{Hod}}^{\mathrm{ev},-}[d] \quad \text { and } \quad \mathrm{H}_{\mathrm{Hod}}^{\mathrm{odd}}(X)[d]_{\mathbb{R}}=\mathrm{H}_{\mathrm{Hod}}^{\mathrm{odd},+}[d] \oplus \mathrm{H}_{\mathrm{Hod}}^{\text {odd, }}[d] .
$$

In order to obtain detailed information about these decompositions, we shall need to compare $\left(\mathrm{H}_{\mathrm{Hod}}^{\mathrm{ev}}(X)[d], \sigma^{\mathrm{ev}}\right)$ and $\left(\mathrm{H}_{\mathrm{Hod}}^{\text {odd }}(X)[d], \sigma^{\text {odd }}\right)$ with the de Rham hypercohomology

$$
\mathrm{H}_{\mathrm{dR}}^{\bullet}(X)[d]=\mathrm{H}^{\bullet}\left(X, \Omega_{X}^{\bullet}[d]\right)=\bigoplus_{t} \mathrm{H}^{t}\left(X, \Omega_{X}^{\bullet}[d]\right)
$$

endowed with the following $G$-invariant form $\tau$.

By (3.2), the pairings $\Omega_{X / \mathbb{R}}^{i} \otimes \Omega_{X / \mathbb{R}}^{j} \rightarrow \Omega_{X / \mathbb{R}}^{i+j}$ give a morphism $\Omega_{X}^{\bullet} \otimes^{\mathbf{L}} \Omega_{X}^{\bullet} \rightarrow \Omega_{X}^{\bullet}$ in the derived category of sheaves of $\mathbb{R}$-vector spaces on $X$. This morphism gives an $\mathbb{R}$-bilinear map

$$
T^{p}: \mathrm{H}^{p}\left(X, \Omega_{X / \mathbb{R}}^{\bullet}[d]\right) \times \mathrm{H}^{-p}\left(X, \Omega_{X / \mathbb{R}}^{\bullet}[d]\right) \rightarrow \mathrm{H}^{0}\left(X, \Omega_{X / \mathbb{R}}^{\bullet}[2 d]\right)
$$

when we identify $\mathrm{H}^{ \pm p}\left(X, \Omega_{X / \mathbb{R}}^{\bullet}[d]\right)$ with $\mathrm{H}^{ \pm p+d}\left(X, \Omega_{X / \mathbb{R}}^{\bullet}\right)$ and $\mathrm{H}^{2 d}\left(X, \Omega_{X / \mathbb{R}}^{\bullet}\right)$ with $\mathrm{H}^{0}\left(X, \Omega_{X / \mathbb{R}}^{\bullet}[2 d]\right)$. Regard $\Omega_{X / \mathbb{R}}^{d}$ as a complex concentrated in dimension 0 . There is then a morphism $\Omega_{X / \mathbb{R}}^{d}[d] \rightarrow$ $\Omega_{X / \mathbb{R}}^{\bullet}[2 d]$ giving a homomorphism

$$
\mathrm{H}^{d}\left(X, \Omega_{X / \mathbb{R}}^{d}\right)=\mathrm{H}^{0}\left(X, \Omega_{X / \mathbb{R}}^{d}[d]\right) \rightarrow \mathrm{H}^{0}\left(X, \Omega_{X / \mathbb{R}}^{\bullet}[2 d]\right) .
$$

By flat base change,

$$
\mathrm{H}_{\mathrm{dR}}^{2 d}(X(\mathbb{C}), \mathbb{C})=_{\text {def }} \mathrm{H}^{0}\left(X(\mathbb{C}), \Omega_{X(\mathbb{C}) / \mathbb{C}}^{\bullet}[2 d]\right)=\mathbb{C} \otimes \mathrm{H}^{0}\left(X, \Omega_{X / \mathbb{R}}^{\bullet}[2 d]\right) .
$$

It follows that (3.11) is an isomorphism because it becomes an isomorphism after tensoring with $\mathbb{C}$ over $\mathbb{R}$ by the Hodge decomposition [GH78, p. 448]. The isomorphism $\mathbb{C} \rightarrow \Omega_{X(\mathbb{C}) / \mathbb{C}}^{\bullet}$ in the derived category gives the Betti to de Rham comparison isomorphism,

$$
\mathrm{H}^{p}(X(\mathbb{C}), \mathbb{C})=\mathrm{H}^{p}\left(X(\mathbb{C}), \Omega_{X(\mathbb{C}) / \mathbb{C}}^{\bullet}\right.
$$

for all $p$. The Dolbeault isomorphism is

$$
\mathbb{C} \otimes_{\mathbb{R}} \mathrm{H}^{p}\left(X, \Omega_{X / \mathbb{R}}^{q}\right)=\mathrm{H}^{p}\left(X(\mathbb{C}), \Omega_{X(\mathbb{C}) / \mathbb{C}}^{q}\right)=\mathrm{H}_{\frac{q}{\partial}}^{q, p}(X(\mathbb{C}))
$$

for all $p$ and $q$, where the definition of $\mathrm{H}_{\bar{\partial}}^{q, p}(X(\mathbb{C}))$ is recalled in the paragraph prior to Proposition 3.4 below. Setting $p=q=d$, there is a non-zero $\mathbb{C}$-linear map

$$
|G|^{-1} \operatorname{Tr}: \mathrm{H}_{\bar{\partial}}^{d, d}(X(\mathbb{C})) \rightarrow \mathbb{C}
$$

defined by

$$
\omega \rightarrow \frac{i^{d}}{(2 \pi)^{d} d !|G|} \int_{X} \omega
$$

for $(d, d)$-forms $\omega$. The composition of this map with the Dolbeault isomorphism gives an isomorphism

$$
|G|^{-1} \operatorname{Tr}: \mathrm{H}^{d}\left(X, \Omega_{X / \mathbb{R}}^{d}\right) \rightarrow \mathbb{R}
$$

in which $\operatorname{Tr}$ is the Serre duality morphism; for more details see $\S 6.2$.

We conclude that (3.11), (3.15) and (3.16) give an $\mathbb{R}$-linear map

$$
\mathrm{H}^{0}\left(X, \Omega_{X / \mathbb{R}}^{d}[d]\right)=\mathrm{H}^{0}\left(X, \Omega_{X / \mathbb{R}}^{\bullet}[2 d]\right) \rightarrow \mathbb{R} .
$$

Composing $T^{p}$ in (3.10) with this map gives an $\mathbb{R}$-valued bilinear form

$$
t^{p}: \mathrm{H}^{p}\left(X, \Omega_{X / \mathbb{R}}^{\bullet}[d]\right) \times \mathrm{H}^{-p}\left(X, \Omega_{X / \mathbb{R}}^{\bullet}[d]\right) \rightarrow \mathbb{R} .
$$

As $d$ is even, the map $t^{0}$ is symmetric (see below); note also that if $x \in \mathrm{H}^{p}\left(X, \Omega_{X / \mathbb{R}}^{\bullet}[d]\right), y \in$ $\mathrm{H}^{-p}\left(X, \Omega_{X / \mathbb{R}}^{\bullet}[d]\right)$, then

$$
t^{p}(x, y)=(-1)^{p} t^{-p}(y, x)
$$




\section{T. Chinburg, G. Pappas and M. Taylor}

which again of course agrees with the commutation rule (3.7). Hence, as per the construction in $\S 3.1$, we may then form the symmetrized duality maps $\tau^{p}$ for all $p$ by defining

$$
\tau^{p}(x, y)=\tau^{-p}(y, x)=t^{p}(x, y) \text { for } p \leqslant 0 .
$$

These pairings give a symmetric non-degenerate $G$-equivariant pairing

$$
\tau=\bigoplus_{p} \tau_{p}: \mathrm{H}_{\mathrm{dR}}^{\bullet}(X)[d] \times \mathrm{H}_{\mathrm{dR}}^{\bullet}(X)[d] \rightarrow \mathbb{R}
$$

on

$$
\mathrm{H}_{\mathrm{dR}}^{\bullet}(X)[d]=\bigoplus_{p<0}\left(\mathrm{H}^{p}\left(X, \Omega_{X / \mathbb{R}}^{\bullet}[d]\right) \oplus \mathrm{H}^{-p}\left(X, \Omega_{X / \mathbb{R}}^{\bullet}[d]\right)\right) \oplus \mathrm{H}^{0}\left(X, \Omega_{X / \mathbb{R}}^{\bullet}[d]\right) .
$$

We write $\Omega_{X}^{\bullet<m}$ respectively $\Omega_{X}^{\bullet \geqslant m}$ for the complex

$$
\begin{gathered}
\mathcal{O}_{X} \stackrel{d}{\rightarrow} \Omega_{X / \mathbb{R}}^{1} \stackrel{d}{\rightarrow} \cdots \stackrel{d}{\rightarrow} \Omega_{X / \mathbb{R}}^{m-1}, \\
\Omega_{X / \mathbb{R}}^{m} \stackrel{d}{\rightarrow} \cdots \stackrel{d}{\rightarrow} \Omega_{X / \mathbb{R}}^{d},
\end{gathered}
$$

where the term $\mathcal{O}_{X}$ (respectively $\Omega_{X / \mathbb{R}}^{m}$ ) is placed in degree 0 (respectively degree $m$ ). We then consider the exact sequence of complexes

$$
0 \rightarrow \Omega_{X / \mathbb{R}}^{\bullet \geqslant m}[d] \rightarrow \Omega_{X / \mathbb{R}}^{\bullet}[d] \rightarrow \Omega_{X / \mathbb{R}}^{\bullet<m}[d] \rightarrow 0
$$

and we let $F^{-m+d / 2}$ denote the image of $\mathrm{H}^{\bullet}\left(X, \Omega_{X}^{\bullet} \geqslant m[d]\right)$ in $\mathrm{H}^{\bullet}\left(X, \Omega_{X}^{\bullet}[d]\right)=\mathrm{H}_{\mathrm{dR}}^{\bullet}(X)[d]$ under the natural map induced by $\Omega_{X / \mathbb{R}}^{\bullet \geqslant m} \hookrightarrow \Omega_{X / \mathbb{R}^{\bullet}}^{\bullet}$ Note that by the degeneration of the Hodge spectral sequence we know that in fact $\mathrm{H}^{\bullet}\left(X, \Omega_{X / \mathbb{R}}^{\bullet \geqslant m}[d]\right)$ injects into $\mathrm{H}^{\bullet}\left(X, \Omega_{X / \mathbb{R}}^{\bullet}[d]\right)$.

Theorem 3.3. The quadratic space $\left(H_{\mathrm{dR}}^{\bullet}(X)[d], \tau\right)$, when endowed with the filtration $\left\{F^{i}\right\}_{i}$, is a filtered quadratic space, as defined in Lemma 2.16. There is an isomorphism of $\mathbb{R}[G]$-quadratic modules

$$
\left(H_{\mathrm{dR}}^{\bullet}(X)[d], \tau\right) \cong\left(H^{d / 2}\left(\Omega_{X}^{d / 2}\right), \sigma_{d / 2, d / 2}\right) \oplus \operatorname{Hyp}\left(\bigoplus_{i<d / 2} H^{i}\left(\Omega_{X}^{d / 2}\right)\right) \oplus \operatorname{Hyp}\left(H^{\bullet}\left(\Omega_{X}^{\bullet>d / 2}\right)\right),
$$

where we abbreviate $\mathrm{H}^{j}(X, \mathcal{F})$ (respectively $\mathrm{H}^{\bullet}(X, \mathcal{F})$ ) by $\mathrm{H}^{j}(\mathcal{F})$ (respectively $\mathrm{H}^{\bullet}(\mathcal{F})$ ) if $\mathcal{F}$ is a sheaf or complex of sheaves on $X$.

Proof. Let $V=\mathrm{H}_{\mathrm{dR}}^{\bullet}(X)[d]$ and let $\sigma=\tau$ in Lemma 2.16. In the definition of $\tau$ in (3.21), $\mathrm{H}^{-p}\left(X, \Omega_{X}^{\bullet}[d]\right)$ contains $\mathrm{H}^{-p}\left(X, \Omega_{X}^{\bullet \geqslant m}[d]\right)=\mathrm{H}^{-p+d}\left(X, \Omega_{X}^{\bullet \geqslant m}\right)$, which is the summand of $F^{-m+d / 2}$ in degree $-p$. From (3.10) we see that the pairing

$$
\mathrm{H}^{\bullet}\left(X, \Omega_{X}^{\bullet \geqslant m}[d]\right) \times \mathrm{H}^{\bullet}\left(X, \Omega_{X}^{\bullet \geqslant d-m}[d]\right) \rightarrow \mathbb{R}
$$

which results from $\tau$ factors through

$$
\tau_{m}^{\prime}: \frac{\mathrm{H}^{\bullet}\left(X, \Omega_{X}^{\bullet \geqslant m}[d]\right)}{\mathrm{H}^{\bullet}\left(X, \Omega_{X}^{\bullet \geqslant m+1}[d]\right)} \times \frac{\mathrm{H}^{\bullet}\left(X, \Omega_{X}^{\bullet \geq d-m}[d]\right)}{\mathrm{H}^{\bullet}\left(X, \Omega_{X}^{\bullet \geq d-m+1}[d]\right)} \rightarrow \mathbb{R} .
$$

By Lemma 2.16, it will suffice to show that $\tau_{m}^{\prime}$ is perfect for all $m$. From the decomposition of the Hodge to de Rham spectral sequence we have

$$
\frac{\mathrm{H}^{-p}\left(X, \Omega_{X}^{\bullet \geqslant m}[d]\right)}{\mathrm{H}^{-p}\left(X, \Omega_{X}^{\bullet \geqslant m+1}[d]\right)} \cong \mathrm{H}^{-p+d}\left(X, \Omega_{X}^{m}[-m]\right)
$$

for all $p$ and $m$. Thus $\tau_{m}^{\prime}$ induces forms

$$
\tau_{m}^{n}: \mathrm{H}^{n+m}\left(X, \Omega_{X}^{m}[-m]\right) \times \mathrm{H}^{2 d-n-m}\left(X, \Omega_{X}^{d-m}[-(d-m)]\right) \rightarrow \mathbb{R}
$$




\section{Pfaffians AND Hodge DiscRiminants}

with $n+m=-p+d$. We now claim that under the above isomorphisms the forms $\tau_{m}^{n}$ agree with the pairings $\sigma_{n, m}$. The definition of $\tau_{m}^{n}$ uses the Dolbeault map (3.15) defined via integration, so we will give a transcendental proof of this comparison after tensoring with $\mathbb{C}$ over $\mathbb{R}$. The intersection pairing on complex Betti cohomology agrees with that on complex de Rham cohomology (see Theorem 3.7 below), while the intersection pairing on Betti cohomology agrees with the wedge product pairing on Dolbeault cohomology by [GH78, p. 59]. Thus it will suffice to show the following result, which is also needed in $\S 6.2$, and which is stated up to sign in [GH78, p. 102]. Since sign information is crucial for us, and we have not found a suitable reference for this in the literature, we will give a proof.

Let $\mathcal{A}_{X(\mathbb{C})}^{p, \bullet}$ be the Dolbeault complex of $\left[\mathrm{GH78}\right.$, p. 448] having degree $q$ term $\mathcal{A}_{X(\mathbb{C})}^{p, q}$ and differential $\bar{\partial}$. Define $\mathcal{A}_{X(\mathbb{C})}^{p, \bullet}\langle-p\rangle$ to be complex having $\mathcal{A}_{X(\mathbb{C})}^{p, q}$ in degree $p+q$ and differential $\bar{\partial}$; this is the same as the result of multiplying all the differentials of $\mathcal{A}_{X(\mathbb{C})}^{p, \bullet}[-p]$ by $(-1)^{p}$. The Dolbeault resolution $\Omega_{X(\mathbb{C})}^{p} \rightarrow \mathcal{A}_{X(\mathbb{C})}^{p, \bullet}$ gives a resolution

$$
\Omega_{X(\mathbb{C})}^{p}[-p] \rightarrow \mathcal{A}_{X(\mathbb{C})}^{p, \bullet}\langle-p\rangle .
$$

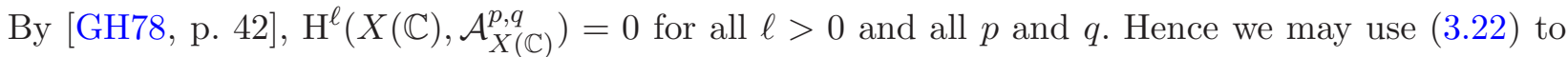
fix the Dolbeault isomorphism

$$
\mathrm{H}^{p+q}\left(X(\mathbb{C}), \Omega_{X(\mathbb{C})}^{p}[-p]\right)=\mathrm{H}^{p+q}\left(X(\mathbb{C}), \mathcal{A}_{X(\mathbb{C})}^{p, \bullet}\langle-p\rangle\right)=\frac{\mathrm{Z}_{\bar{\partial}}^{p, q}(X(\mathbb{C}))}{\mathrm{B}_{\bar{\partial}}^{p, q}(X(\mathbb{C}))}=\mathrm{H}_{\bar{\partial}}^{p, q}(X(\mathbb{C})) .
$$

Proposition 3.4. The wedge product of forms gives the following commutative diagram of pairings.

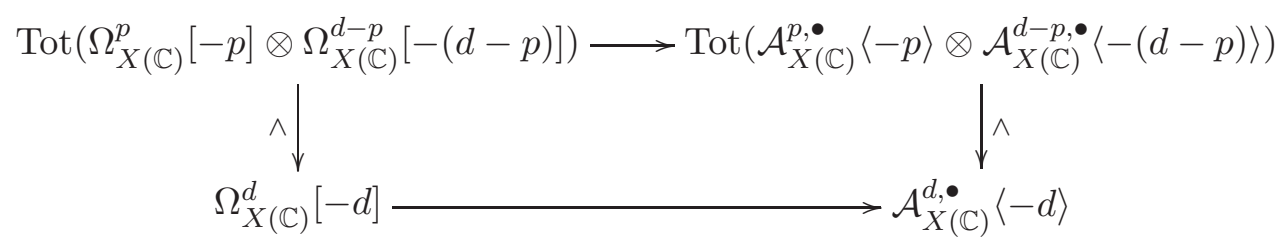

This gives in cohomology the commutative diagram

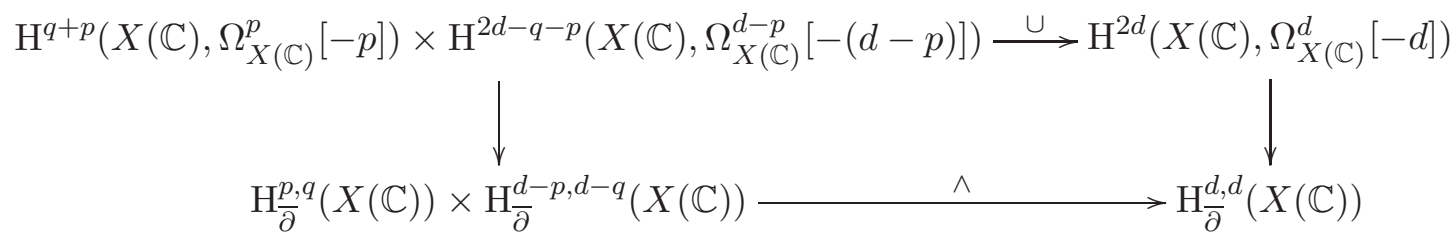

in which the vertical maps are Dolbeault isomorphisms and $\cup$ in the top row is $\sigma_{q, p}$.

Before proving the proposition, we first note that it will complete the proof of the theorem. Indeed, by Lemma 2.16, we know that

$$
\begin{aligned}
\left(\mathrm{H}_{\mathrm{dR}}^{\bullet}(X)[d, \tau)\right. & \cong\left(\bigoplus_{i} \mathrm{H}^{i}\left(\Omega_{X}^{d / 2}\right), \tau_{d / 2}^{\prime}\right) \oplus \operatorname{Hyp}\left(\mathrm{H}^{\bullet}\left(\Omega_{X}^{\bullet>d / 2}\right)\right) \\
& \cong\left(\mathrm{H}^{d / 2}\left(\Omega_{X}^{d / 2}\right), \tau_{d / 2}^{d / 2}\right) \oplus \operatorname{Hyp}\left(\bigoplus_{i<d / 2} \mathrm{H}^{i}\left(\Omega_{X}^{d / 2}\right)\right) \oplus \operatorname{Hyp}\left(\mathrm{H}^{\bullet}\left(\Omega_{X}^{\bullet>d / 2}\right)\right) .
\end{aligned}
$$

However, by the above discussion together with the proposition, we know that $\tau_{d / 2}^{d / 2}$ is equal to $\sigma_{d / 2, d / 2}$ and the result will now follow.

Proof of Proposition 3.4. We first check that the wedge product of forms gives a well-defined morphism of complexes on the right vertical side of (3.24). Let $D^{p+q}$ be the $(p+q)$ th boundary map 


\section{T. Chinburg, G. Pappas and M. Taylor}

of $\mathcal{A}_{X(\mathbb{C})}^{p, \bullet}\langle-p\rangle$ and let $D^{\prime d-p+q^{\prime}}$ be the $\left(d-p+q^{\prime}\right)$ th boundary map of $\mathcal{A}_{X(\mathbb{C})}^{d-p, \bullet}\langle-(d-p)\rangle$. These are identified with $\bar{\partial}: \mathcal{A}_{X(\mathbb{C})}^{p, q} \rightarrow \mathcal{A}_{X(\mathbb{C})}^{p, q+1}$ and $\bar{\partial}: \mathcal{A}_{X(\mathbb{C})}^{d-p, q^{\prime}} \rightarrow \mathcal{A}_{X(\mathbb{C})}^{d-p, q^{\prime}+1}$, respectively. By (3.2), the boundary map of degree $(p+q)+\left(d-p+q^{\prime}\right)=d+q+q^{\prime}$ for the summand $\left(\mathcal{A}_{X(\mathbb{C})}^{p, \bullet}\langle-p\rangle\right)^{p+q} \otimes\left(\mathcal{A}_{X(\mathbb{C})}^{d-p, \bullet}\langle-p\rangle\right)^{d-p+q^{\prime}}$ of $\operatorname{Tot}\left(\mathcal{A}_{X(\mathbb{C})}^{p, \bullet}\langle-p\rangle \otimes \mathcal{A}_{X(\mathbb{C})}^{d-p, \bullet}\langle-(d-p)\rangle\right)$ is given by

$$
\left(D^{(p+q)} \otimes \mathrm{Id}\right)+(-1)^{p+q}\left(\mathrm{Id} \otimes D^{\left(d-p+q^{\prime}\right)}\right) .
$$

In the notation of [GH78, p. 24], this differential sends

$$
\left(\phi(z) d z_{I} \wedge d \bar{z}_{J}\right) \otimes\left(\psi(z) d z_{I^{\prime}} \wedge d \bar{z}_{J^{\prime}}\right)
$$

to

$$
\begin{aligned}
& \left(\sum_{j} \frac{\partial \phi(z)}{\partial \bar{z}_{j}} d \bar{z}_{j} \wedge d z_{I} \wedge d \bar{z}_{J}\right) \otimes\left(\psi(z) d z_{I^{\prime}} \wedge d \bar{z}_{J^{\prime}}\right) \\
& \quad+(-1)^{p+q}\left(\phi(z) d z_{I} \wedge d \bar{z}_{J}\right) \otimes\left(\sum_{j} \frac{\partial \psi(z)}{\partial \bar{z}_{j}} d \bar{z}_{j} \wedge d z_{I^{\prime}} \wedge d \bar{z}_{J^{\prime}}\right) .
\end{aligned}
$$

The image of this sum under the wedge product morphism on the right vertical side of (3.24) is

$$
\sum_{j}\left(\frac{\partial \phi(z)}{\partial \bar{z}_{j}} \psi(z)+\phi(z) \frac{\partial \psi(z)}{\partial \bar{z}_{j}}\right) d \bar{z}_{j} \wedge d z_{I} \wedge d \bar{z}_{J} \wedge d z_{I^{\prime}} \wedge d \bar{z}_{J^{\prime}}
$$

since $\# I+\# J=p+q$. The boundary map in degree $d+q+q^{\prime}$ of $\mathcal{A}_{X(\mathbb{C})}^{d, \bullet}\langle-d\rangle$ is identified with $\bar{\partial}: \mathcal{A}_{X(\mathbb{C})}^{d, q+q^{\prime}} \rightarrow \mathcal{A}_{X(\mathbb{C})}^{d, q+q^{\prime}+1}$. By the Leibniz formula, this boundary map sends the image of (3.26) under the right vertical map in (3.24) to the form (3.28). Hence the right side of (3.24) is a morphism of complexes. The fact that (3.25) commutes then becomes a tautology in view of the fact that we normalized the Dolbeault isomorphism (3.23) using the resolution (3.22) entering into the top row of (3.24).

Corollary 3.5. There is a non-canonical $\mathbb{R}[G]$-isometry

$$
\left(\mathrm{H}_{\mathrm{dR}}^{\bullet}(X)[d], \tau\right) \cong\left(\mathrm{H}_{\mathrm{Hod}}^{\bullet}(X)[d], \sigma\right) .
$$

Proof. By the above proposition we know that each of the above quadratic spaces is isometric to the orthogonal sum of $\left(\mathrm{H}^{d / 2}\left(\Omega_{X}^{d / 2}\right), \sigma_{d / 2, d / 2}\right)$ and a hyperbolic space. On the other hand, by the degeneration of the Hodge to de Rham spectral sequence, we know that $\mathrm{H}_{\mathrm{dR}}^{\bullet}(X)$ and $\mathrm{H}_{\mathrm{Hod}}^{\bullet}(X)$ are isomorphic $\mathbb{R}[G]$-modules. Therefore we may conclude that the two hyperbolic spaces are isometric, as required.

\subsection{Betti cohomology}

Throughout this section we shall again suppose that the dimension $d$ of $X$ is even, so that $X(\mathbb{C})$ has real dimension divisible by 4 . Hence the (unmodified) cup-product $c^{d}$ is a non-degenerate symmetric $G$-invariant form on $\mathrm{H}_{B}^{d}(X(\mathbb{C}), \mathbb{R})$ via the map $\mathrm{H}_{B}^{2 d}(X(\mathbb{C}), \mathbb{R}) \rightarrow \mathbb{R}$. By Proposition 2.12 we know that $\mathrm{H}_{B}^{d}(X(\mathbb{C}), \mathbb{R})$ admits a non-canonical decomposition of $\mathbb{R}[G]$-modules

$$
\mathrm{H}_{B}^{d}(X(\mathbb{C}), \mathbb{R})=\mathrm{H}_{B}^{d,+} \oplus \mathrm{H}_{B}^{d,-},
$$

where $\mathrm{H}_{B}^{d,+}$ is a maximal positive definite subspace and $\mathrm{H}_{B}^{d,-}$ is a maximal negative definite subspace of $\mathrm{H}_{B}^{d}(X(\mathbb{C}), \mathbb{R})$ with respect to $c^{d}$.

For $t<d$ we let $c^{t}$ denote the symmetrized $G$-invariant form on $\mathrm{H}_{B}^{t}(X(\mathbb{C}), \mathbb{R}) \oplus \mathrm{H}_{B}^{2 d-t}(X(\mathbb{C}), \mathbb{R})$ induced by the cup-product

$$
\mathrm{H}_{B}^{t}(X(\mathbb{C}), \mathbb{R}) \times \mathrm{H}_{B}^{2 d-t}(X(\mathbb{C}), \mathbb{R}) \rightarrow \mathrm{H}_{B}^{2 d}(X(\mathbb{C}), \mathbb{R}) \rightarrow \mathbb{R}
$$




\section{PfaffiAns AND HodGe DISCRIMINANTS}

as per the construction of $\sigma^{t}$ in $\S 3.1$. Note that the symmetrization here is the same as that used in $\S$ 3.1. Thus for $x \in \mathrm{H}_{B}^{t}(X(\mathbb{C}), \mathbb{R}), y \in \mathrm{H}_{B}^{2 d-t}(X(\mathbb{C}), \mathbb{R})$ and $t<d$ we have

$$
c(y, x)=(-1)^{t} c(x, y) .
$$

For $t<d, c^{t}$ is hyperbolic and by Proposition 2.12 we have a decomposition of $\mathbb{R}[G]$-modules

$$
\mathrm{H}_{B}^{\text {odd }}(X(\mathbb{C}), \mathbb{R})=\mathrm{H}_{B}^{\text {odd }+} \oplus \mathrm{H}_{B}^{\text {odd }-}
$$

into positive and negative subspaces. Applying Proposition 2.12 once again we obtain a decomposition

$$
\mathrm{H}_{B}^{\mathrm{ev}}(X(\mathbb{C}), \mathbb{R})=\mathrm{H}_{B}^{\mathrm{ev}+} \oplus \mathrm{H}_{B}^{\mathrm{ev}-}
$$

where $\mathrm{H}_{B}^{d,+} \subset \mathrm{H}_{B}^{\mathrm{ev}+}$ and $\mathrm{H}_{B}^{d,-} \subset \mathrm{H}_{B}^{\mathrm{ev}-}$.

Furthermore, by Proposition 2.12 and by hyperbolicity, we know that, as $\mathbb{R}[G]$-modules,

$$
\begin{gathered}
\mathrm{H}_{B}^{\mathrm{ev}+} / \mathrm{H}_{B}^{d,+} \cong \mathrm{H}_{B}^{\mathrm{ev}-} / \mathrm{H}_{B}^{d,-} \cong \bigoplus_{t \text { even }, t<d} \mathrm{H}_{B}^{t}(X(\mathbb{C}), \mathbb{R}) \\
\mathrm{H}_{B}^{\text {odd }+} \cong \mathrm{H}_{B}^{\text {odd }-} \cong \bigoplus_{t \text { odd }, t<d} \mathrm{H}_{B}^{t}(X(\mathbb{C}), \mathbb{R})
\end{gathered}
$$

Theorem 3.6. With the above notation and hypotheses, $\mathrm{H}_{B}^{\bullet+}$ and $\mathrm{H}_{B}^{\bullet-}$ are both free virtual $\mathbb{R}[G]$ modules.

Proof. Since $G$ acts freely on $X(\mathbb{C})$, by the Lefschetz fixed point theorem (see for instance [Ver73]) for each $g \in G, g \neq 1$, the virtual character associated to $\mathrm{H}_{B}^{\bullet}(X(\mathbb{C}), \mathbb{R})$ is zero when evaluated on such $g$; thus $\mathrm{H}_{B}^{\bullet}=\mathrm{H}_{B}^{\bullet+}+\mathrm{H}_{B}^{\bullet-}$ is a free virtual $\mathbb{R}[G]$-module.

Similarly we shall show that $\mathrm{H}_{B}^{\bullet+}-\mathrm{H}_{B}^{\bullet-}$ is a free virtual $\mathbb{R}[G]$-module; this will then establish the theorem. To see that $\mathrm{H}_{B}^{\bullet+}-\mathrm{H}_{B}^{\bullet-}$ is free, we recall that by the $G$-signature theorem in [AS68, Theorem 6.12] (see also [Sha78, V.18]), for each non-trivial element $g \in G$, the value of the virtual character of $\mathrm{H}_{B}^{\bullet+}-\mathrm{H}_{B}^{\bullet-}$ evaluated on $g$ is presented in terms of data associated to the fixed point set $X(\mathbb{C})^{g}$. Since $g$ acts without fixed points, it then follows that this virtual character is zero on all such $g$.

\subsection{The comparison isomorphism}

In this section we take $K=\mathbb{R}$ and suppose that $X$ has even dimension.

Theorem 3.7. Let $\langle\iota\rangle=\operatorname{Gal}(\mathbb{C} / \mathbb{R})$. The comparison isomorphism

$$
\mathrm{H}_{\mathrm{dR}}^{\bullet}(X) \otimes_{\mathbb{R}} \mathbb{C} \rightarrow \mathrm{H}_{B}^{\bullet}(X) \otimes_{\mathbb{R}} \mathbb{C}
$$

is an isometry when $\mathrm{H}_{\mathrm{dR}}^{\bullet}(X)$ is endowed with $\sigma$ and $\mathrm{H}_{B}^{\bullet}(X)$ is endowed with $c$. Moreover, under this isomorphism $\mathrm{H}_{B}^{\bullet}(X)$ identifies as an $\mathbb{R}$-subspace of $\mathrm{H}_{\mathrm{dR}}^{\bullet}(X) \otimes_{\mathbb{R}} \mathbb{C}$ which is stable under the action of both $G$ and $\iota$.

Proof. The comparison isomorphism is certainly an isometry when $\mathrm{H}_{\mathrm{dR}}^{\bullet}(X)$ is endowed with the unsymmetrized duality pairing and $\mathrm{H}_{B+}^{\bullet}(X)$ is endowed with the unsymmetrized form coming from the cup-product (see for instance [GH78, p. 59]). From (3.7) and (3.29) we see that the sign changes involved in the symmetrization process agree and so the symmetrized forms are also isometries.

The stability of the image of $\mathrm{H}_{B}^{\bullet}(X)$ under $G$ follows from the functoriality of the comparison isomorphism, and its stability under $\iota$ follows from [Del79, Corollary 1.6, p. 320].

In the next section we shall use the above theorem together with Proposition 2.15 to obtain signature information for de Rham cohomology from knowledge of the signature properties of Betti cohomology. 


\section{T. Chinburg, G. Pappas and M. Taylor}

\section{Archimedean invariants}

Throughout all of this section we shall suppose that $X$ is defined over $\mathbb{R}$. We begin by recalling some detailed formulas for the archimedean $\varepsilon$-constants associated to $X$. We then use these results and the work in the previous section to prove Theorem 1.1.

\subsection{Archimedean $\varepsilon$-constants}

Here we recall a number of results from $[\mathrm{CEPT} 97, \S 5]$. Let $F_{\infty}: X(\mathbb{C}) \rightarrow X(\mathbb{C})$ denote the involution induced by complex conjugation on $X(\mathbb{C})$, the space of complex points of $X$; then $F_{\infty}$ acts on the Betti cohomology $\mathrm{H}_{B}^{i}(X(\mathbb{C}), \mathbb{Q})$ and, for a complex representation $V$ of $G$ with contragredient $V^{*}$, we write $\mathrm{H}_{B+}^{i}\left(V \otimes_{G} X\right)$ (respectively $\mathrm{H}_{B-}^{i}\left(V \otimes_{G} X\right)$ ) for the subspace of $\left(V^{*} \otimes_{\mathbb{Q}} \mathrm{H}_{B}^{i}(X(\mathbb{C}), \mathbb{Q})\right)^{G}$ on which $F_{\infty}$ acts by +1 (respectively -1 ). (For a discussion of the motives $V \otimes_{G} X$ see [CEPT97, $\S 2]$.) We then set

$$
\chi_{ \pm}\left(V \otimes_{G} X\right)=\sum_{i=0}^{2 d}(-1)^{i} \operatorname{dim}_{\mathbb{C}}\left(\mathrm{H}_{B \pm}^{i}\left(V \otimes_{G} X\right)\right)
$$

and we may extend $\chi_{ \pm}\left(V \otimes_{G} X\right)$ to virtual representations since it is additive in $V$.

The archimedean constant $\varepsilon_{\infty}\left(V \otimes_{G} X\right)$ is constructed from the Hodge realization of the real motive $V \otimes_{G} X$ (see for instance [CEPT97, $\left.\S 5\right]$ for the details of the construction of such $\varepsilon$-constants); again it is also additive in $V$ and thus extends to virtual $V$.

Lemma 4.1. Let $W$ be a virtual symplectic complex representation of $G$.

(a) Both $\chi_{ \pm}\left(W \otimes_{G} X\right)$ are even integers.

(b) If $d$ is odd, then $\varepsilon_{\infty}\left(W \otimes_{G} X\right)=1$.

(c) If $d$ is even, then writing \pm for the sign of $(-1)^{d / 2+1}$ we have

$$
\varepsilon_{\infty}\left(W \otimes_{G} X\right)=i^{\chi_{ \pm}\left(W \otimes_{G} X\right)}
$$

and, moreover, if $\operatorname{dim}_{\mathbb{C}}(W)=0$, then $\varepsilon_{\infty}\left(W \otimes_{G} X\right)=i^{\chi+}\left(W \otimes_{G} X\right)=i^{\chi-\left(W \otimes_{G} X\right)}$.

Proof. Part (a) follows from Lemma 2.9, which shows that each $\operatorname{dim}_{\mathbb{C}}\left(\mathrm{H}_{B \pm}^{i}\left(W \otimes_{G} X\right)\right)$ is even; parts (b) and (c) come from [CEPT97, Lemma 5.1.1].

\subsection{Proof of Theorem 1.1}

Let $\theta$ denote a symplectic character of $G$. By Proposition 2.14, we have

$$
\text { sgn.pf }\left(\theta, \sigma, R \Gamma\left(X, \bigoplus_{i} \Omega_{X / \mathbb{R}}^{i}[d-i]\right)\right)=(\sqrt{-1})^{\left(\sum_{t \geqslant 0}(-1)^{t} n_{\theta}^{-}\left(\sigma^{t}\right)\right)}=(\sqrt{-1})^{n_{\theta}^{-}(\sigma)} .
$$

In this section we complete the proof of Theorem 1.1 by showing that, for an arbitrary virtual symplectic $\mathbb{C}[G]$ representation $W$ of $G$,

$$
(-1)^{\delta(Y) \theta(1) / 2} \varepsilon_{\infty}\left(W \otimes_{G} X\right)=i^{n_{W}^{-}(\sigma)} .
$$

By writing $W=(W-\operatorname{dim}(W))+\operatorname{dim}(W)$ and using additivity we reduce our proof of (4.2) to two cases: the case where $W$ has dimension 0 , and the case where $W$ is two copies of the trivial representation $T$ of $G$.

Throughout the proof we will set $P_{0}=\mathrm{H}_{\mathrm{Hod}}^{\mathrm{ev}}\left(\right.$ respectively $P_{1}=\mathrm{H}_{\mathrm{Hod}}^{\text {odd }}$ ), endowed with $\sigma^{\text {ev }}$ (respectively $\left.\sigma^{\text {odd }}\right)$. Suppose first that $d$ is odd. Then $\left(P_{0}, \sigma^{\text {ev }}\right)$ and $\left(P_{1}, \sigma^{\text {odd }}\right)$ are hyperbolic by Proposition 3.1. If $W$ has dimension 0, then both terms in (4.2) are 1 by Lemma 4.1 and Proposition 2.15(b). Suppose now that $W$ is isomorphic to two copies of $T$. Since we have supposed $d$ is odd, $\delta(Y)=\chi(Y) / 2$ by definition. By Lemma 4.1(b) we know that $\varepsilon_{\infty}\left(W \otimes_{G} X\right)=1$. 


\section{Pfaffians And Hodge Discriminants}

Because the $\left(P_{i}, \sigma_{i}\right)$ are hyperbolic in this case, Proposition 2.15(c) with $V=P$ shows that $n_{W}^{-}(\sigma)=\operatorname{dim}\left(P_{W}^{-}\right)=\operatorname{dim}\left(P_{T}\right)=\chi(Y)$ and so the equality (4.2) has been shown to hold.

We now suppose that $d$ is even. Let $V_{0}=\mathrm{H}_{\mathrm{B}}^{\mathrm{ev}}$ (respectively $V_{1}=\mathrm{H}_{\mathrm{B}}^{\text {odd }}$ ), endowed with $c^{\mathrm{ev}}$ (respectively $c^{\text {odd }}$ ). We note that by Theorem 3.7 the general conditions of Proposition 2.15 are indeed satisfied. Suppose $W$ has dimension 0. By Theorem 3.6, $V_{0}^{ \pm}-V_{1}^{ \pm}$is $\mathbb{R}[G]$-free, and so by Proposition 2.15(b) we know that

$$
n_{W}^{-}(\sigma) \equiv \operatorname{dim}\left(P_{W}^{0,-}\right)-\operatorname{dim}\left(P_{W}^{1,-}\right) \equiv \chi_{-}\left(W \otimes_{G} X\right) \bmod 4
$$

and the equality in this case now follows from Lemma 4.1(c).

To conclude we consider the case where $W$ is two copies of $T$ and $d$ is even. We write $\chi^{ \pm}(Y)=$ $\operatorname{dim}\left(V_{T}^{0, \pm}\right)-\operatorname{dim}\left(V_{T}^{1, \pm}\right)$. By Lemma 4.1(c) we need to show that

$$
\operatorname{dim}\left(P_{W}^{-}\right) \equiv 2 \chi_{ \pm}(Y)-2 \chi^{ \pm}(Y) \bmod 4
$$

where \pm is given by $(-1)^{d / 2+1}$. From Proposition 2.15 (c) we have the congruence

$$
\operatorname{dim}\left(P_{W}^{-}\right) \equiv 2 \chi^{-}(Y)-2 \chi_{-}(Y) \bmod 4 .
$$

To conclude we consider separately the two cases $d \equiv 0,2 \bmod 4$.

Case 1: $d \equiv 2 \bmod 4$. In this case by (4.5) and the fact that $2 \chi^{ \pm}(Y) \equiv-2 \chi^{ \pm}(Y) \bmod 4$ we have to show the congruence

$$
2 \chi^{-}(Y)-2 \chi_{-}(Y) \equiv 2 \chi^{+}(Y)+2 \chi_{+}(Y) \bmod 4,
$$

which is clear since

$$
\chi_{+}(Y)+\chi_{-}(Y)=\chi(Y)=\chi^{+}(Y)+\chi^{-}(Y) .
$$

Case 2: $\quad d \equiv 0 \bmod 4$. This follows at once since we have to show the obvious congruence

$$
2 \chi^{-}(Y)+2 \chi_{-}(Y) \equiv 2 \chi^{-}(Y)-2 \chi_{-}(Y) \bmod 4 .
$$

\section{Class groups}

We begin this section by giving the definition of the symplectic hermitian class group, and we also briefly recall the definition of the equivariant Arakelov class group; for full details on the latter see [CPT02]. We then go on to explain how to associate a hermitian Euler characteristic (respectively an Arakelov Euler characteristic) to a perfect $\mathbb{Z}[G]$ complex with suitable symmetric forms (respectively metrics) on their cohomology.

\subsection{Definition of class groups}

Recall that $R_{G}$ denotes the group of complex virtual characters of $G$, and $R_{G}^{\mathrm{s}}$ is the subgroup of virtual symplectic characters. Let $\overline{\mathbb{Q}}$ be the algebraic closure of $\mathbb{Q}$ in $\mathbb{C}$, and define $\Omega=\operatorname{Gal}(\overline{\mathbb{Q}} / \mathbb{Q})$. Define $J_{f}$ (respectively $J_{\infty}$ ) to be the group of finite ideles (respectively the archimedean ideles) of $\overline{\mathbb{Q}}$. Thus $J_{f}$ is the direct limit of the finite idele groups of all algebraic number fields $E$ in $\overline{\mathbb{Q}}$, and

$$
J_{\infty}=\lim _{E \subset \overline{\mathbb{Q}}}\left(E \otimes_{\mathbb{Q}} \mathbb{R}\right)^{\times} .
$$

The idele group of $\overline{\mathbb{Q}}$ is $J=J_{f} \times J_{\infty}$.

Let $\widehat{\mathbb{Z}}=\prod_{p} \mathbb{Z}_{p}$ denote the ring of integral finite ideles of $\mathbb{Z}$. For $x \in \widehat{\mathbb{Z}}[G]^{\times}$, the element $\operatorname{Det}(x) \in$ $\operatorname{Hom}_{\Omega}\left(R_{G}, J_{f}\right)$ is defined by the rule that, for a representation $T$ of $G$ with character $\psi$,

$$
\operatorname{Det}(x)(\psi)=\operatorname{det}(T(x)) \text {; }
$$




\section{T. Chinburg, G. Pappas and M. Taylor}

the group of all such homomorphisms is denoted by

$$
\operatorname{Det}\left(\widehat{\mathbb{Z}}[G]^{\times}\right) \subseteq \operatorname{Hom}_{\Omega}\left(R_{G}, J_{f}\right) .
$$

More generally, for $n>1$ we can form the group $\operatorname{Det}\left(G L_{n}(\widehat{\mathbb{Z}}[G])\right)$. Because each group ring $\mathbb{Z}_{p}[G]$ is semi-local, we have from [Tay $84, \S 1.2 .6$ ] the equality

$$
\operatorname{Det}\left(G L_{n}(\widehat{\mathbb{Z}}[G])\right)=\operatorname{Det}\left(\widehat{\mathbb{Z}}[G]^{\times}\right) .
$$

Recall that by the Hasse-Schilling norm theorem

$$
\operatorname{Det}\left(\mathbb{Q}[G]^{\times}\right)=\operatorname{Hom}_{\Omega}^{+}\left(R_{G}, \overline{\mathbb{Q}}^{\times}\right),
$$

where the right-hand expression denotes Galois equivariant homomorphisms whose values on $R_{G}^{\mathrm{s}}$ are all totally positive. We then have a diagonal map

$$
\Delta: \operatorname{Hom}_{\Omega}^{+}\left(R_{G}, \overline{\mathbb{Q}}^{\times}\right) \rightarrow \operatorname{Hom}_{\Omega}\left(R_{G}, J_{f}\right) \times \operatorname{Hom}\left(R_{G}, \mathbb{R}_{>0}\right)
$$

where $\Delta(f)=f \times|f|$. Given a homomorphism $f$ on $R_{G}$, we shall write $f^{\text {s }}$ for the restriction of $f$ to $R_{G}^{\mathrm{s}}$; in particular we write

$$
\Delta^{\mathrm{s}}: \operatorname{Hom}_{\Omega}^{+}\left(R_{G}^{\mathrm{s}}, \overline{\mathbb{Q}}^{\times}\right) \rightarrow \operatorname{Hom}_{\Omega}\left(R_{G}^{\mathrm{s}}, J_{f}\right) \times \operatorname{Hom}\left(R_{G}^{\mathrm{s}}, \mathbb{R}_{>0}\right)
$$

for the restriction of $\Delta$ to $R_{G}^{\mathrm{s}}$, so that

$$
\Delta^{\mathrm{s}}\left(f^{\prime}\right)=f^{\prime} \times\left|f^{\prime}\right|=f^{\prime} \times f^{\prime} .
$$

Definition 5.1. The group of symplectic hermitian classes $\mathrm{H}^{\mathrm{s}}(\mathbb{Z}[G])$ is defined to be the quotient group

$$
\mathrm{H}^{\mathrm{s}}(\mathbb{Z}[G])=\frac{\operatorname{Hom}_{\Omega}\left(R_{G}^{\mathrm{s}}, J_{f}\right) \times \operatorname{Hom}\left(R_{G}^{\mathrm{s}}, \mathbb{R}^{\times}\right)}{\operatorname{Im}\left(\Delta^{\mathrm{s}}\right) \cdot\left(\operatorname{Det}^{\mathrm{s}}\left(\widehat{\mathbb{Z}}[G]^{\times}\right) \times 1\right)},
$$

where $\operatorname{Det}^{\mathrm{s}}\left(\widehat{\mathbb{Z}}[G]^{\times}\right)$denotes the restriction of $\operatorname{Det}\left(\widehat{\mathbb{Z}}[G]^{\times}\right)$to hermitian class group $\mathrm{H}^{\mathrm{s}}(\mathbb{Z}[G])$ is slightly different from the hermitian class group $\operatorname{HCl}(\mathbb{Z}[G])$ used in [CPT03] and [Frö84]. There is a natural map between these two class groups. For details see Appendix A.)

Definition 5.2. Suppose $O_{L}$ is the ring of integers of a number field $L$. Let $\Omega_{L}=\operatorname{Gal}(\bar{L} / L)$. Let $J(\bar{L})$ (respectively $J_{f}(\bar{L})$ ) be the ideles (respectively finite ideles) of $\bar{L}$. Define $U\left(O_{L}[G]\right.$ ) (respectively $\left.U_{f}\left(O_{L}[G]\right)\right)$ to be the multiplicative group of unit ideles (respectively finite unit ideles) of the group ring $O_{L}[G]$. Let $\operatorname{Det}^{\mathrm{s}}\left(U\left(O_{L}[G]\right)\right)$ (respectively $\operatorname{Det}^{\mathrm{s}}\left(U_{f}\left(O_{L}[G]\right)\right)$ ) be the subgroup of $\operatorname{Hom}_{\Omega_{F}}\left(R_{G}^{\mathrm{s}}, J(\bar{L})\right)$ (respectively $\operatorname{Hom}_{\Omega_{F}}\left(R_{G}^{\mathrm{s}}, J_{f}(\bar{L})\right)$ ) formed by the restrictions to $R_{G}^{\mathrm{s}}$ of elements of $\operatorname{Det}\left(U\left(O_{L}[G]\right)\right)$ (respectively $\operatorname{Det}\left(U_{f}\left(O_{L}[G]\right)\right)$ ). The adelic hermitian class group $\operatorname{AdHCL}\left(O_{L}[G]\right)$ is defined by

$$
\operatorname{AdHCL}\left(O_{L}[G]\right)=\frac{\operatorname{Hom}_{\Omega_{L}}\left(R_{G}^{\mathrm{s}}, J(\bar{L})\right)}{\operatorname{Det}^{\mathrm{s}}\left(U\left(O_{L}[G]\right)\right)} .
$$

The finite adelic hermitian class group $\operatorname{AdHCL}_{f}\left(O_{L}[G]\right)$ is defined by

$$
\operatorname{AdHCL}_{f}\left(O_{L}[G]\right)=\frac{\operatorname{Hom}_{\Omega_{L}}\left(R_{G}^{\mathrm{s}}, J_{f}(\bar{L})\right)}{\operatorname{Det}^{\mathrm{s}}\left(U_{f}\left(O_{L}[G]\right)\right)} .
$$

Recall from [CPT02, Definition 3.2] that the group of Arakelov classes is defined as

$$
\mathrm{A}(\mathbb{Z}[G])=\frac{\operatorname{Hom}_{\Omega}\left(R_{G}, J_{f}\right) \times \operatorname{Hom}\left(R_{G}, \mathbb{R}_{>0}\right)}{\operatorname{Im}(\Delta) \cdot\left(\operatorname{Det}\left(\widehat{\mathbb{Z}}[G]^{\times}\right) \times 1\right)}
$$

and that the group of symplectic Arakelov classes (see [CPT02, Definition 4.1]) is

$$
\mathrm{A}^{\mathrm{s}}(\mathbb{Z}[G])=\frac{\operatorname{Hom}_{\Omega}\left(R_{G}^{\mathrm{s}}, J_{f}\right) \times \operatorname{Hom}\left(R_{G}^{\mathrm{s}}, \mathbb{R}_{>0}\right)}{\operatorname{Im}\left(\Delta^{\mathrm{s}}\right) \cdot\left(\operatorname{Det}^{\mathrm{s}}\left(\widehat{\mathbb{Z}}[G]^{\times}\right) \times 1\right)}
$$




\section{PfaffiAns AND HodGe DISCRIMINANTS}

Remark 5.3. Firstly, from the above descriptions, we see that $\mathrm{A}^{\mathrm{s}}(\mathbb{Z}[G])$ is naturally a subgroup of $\mathrm{H}^{\mathrm{s}}(\mathbb{Z}[G])$. Secondly, from [Frö83, Lemma 2.1, p. 60], we note that, since all symplectic characters are real-valued, there is a natural isomorphism $\operatorname{Hom}_{\Omega}\left(R_{G}^{\mathrm{s}}, J_{\infty}\right) \cong \operatorname{Hom}\left(R_{G}^{\mathrm{s}}, \mathbb{R}^{\times}\right)$induced by the inclusion $\overline{\mathbb{Q}} \subset \mathbb{C}$.

\subsection{Rational classes and signature classes}

Let $-1_{\infty}$ denote the idele which is 1 at all finite primes and which is -1 at all infinite primes. We consider the two subgroups of

$$
\operatorname{Hom}_{\Omega}\left(R_{G}^{\mathrm{s}}, J\right)=\operatorname{Hom}_{\Omega}\left(R_{G}^{\mathrm{s}}, J_{f}\right) \times \operatorname{Hom}_{\Omega}\left(R_{G}^{\mathrm{s}}, J_{\infty}\right) \cong \operatorname{Hom}_{\Omega}\left(R_{G}^{\mathrm{s}}, J_{f}\right) \times \operatorname{Hom}\left(R_{G}^{\mathrm{s}}, \mathbb{R}^{\times}\right)
$$

given by

$$
\begin{gathered}
\mathrm{R}(\mathbb{Z}[G])=\operatorname{Hom}_{\Omega}\left(R_{G}^{\mathrm{s}}, \mathbb{Q}^{\times}\right) \times 1, \\
\mathrm{~S}_{\infty}(\mathbb{Z}[G])=1 \times \operatorname{Hom}\left(R_{G}^{\mathrm{s}}, \pm 1\right)=\operatorname{Hom}\left(R_{G}^{\mathrm{s}}, \pm 1_{\infty}\right) .
\end{gathered}
$$

Theorem 5.4. The natural map from $\operatorname{Hom}_{\Omega}\left(R_{G}^{\mathrm{s}}, J\right)$ to $\mathrm{H}^{\mathrm{s}}(\mathbb{Z}[G])$ is injective on $\mathrm{R}(\mathbb{Z}[G]) \times \mathrm{S}_{\infty}(\mathbb{Z}[G])$. Thus in the sequel we shall view $\mathrm{R}(\mathbb{Z}[G]) \times \mathrm{S}_{\infty}(\mathbb{Z}[G])$ as a subgroup of $\mathrm{H}^{\mathrm{s}}(\mathbb{Z}[G])$.

Proof. Let $r \times s \in \mathrm{R}(\mathbb{Z}[G]) \times \mathrm{S}_{\infty}(\mathbb{Z}[G])$. We must show that, if $r \times s \in \operatorname{Im}\left(\Delta^{\mathrm{s}}\right) \cdot\left(\operatorname{Det}^{\mathrm{S}}\left(\widehat{\mathbb{Z}}[G]^{\times}\right) \times 1\right)$, then $r=1=s$. Now by the Hasse-Schilling theorem we see immediately that $s$ is positive and hence 1 . We therefore deduce that $r \in \mathrm{R}(\mathbb{Z}[G]) \cap \operatorname{Det}^{\mathrm{s}}\left(\widehat{\mathbb{Z}}[G]^{\times}\right)$, which is known to be trivial by $[\mathrm{CNT} 83$, Proposition 6.1] (see also [Frö84, Theorem 17, p. 190]).

The counterpart for Arakelov classes is the following result, which is shown in [CPT02, $\S 4.4]$.

Theorem 5.5. The natural map from $\operatorname{Hom}_{\Omega}\left(R_{G}^{\mathrm{s}}, J_{f}\right) \times \operatorname{Hom}\left(R_{G}^{\mathrm{s}}, \mathbb{R}_{>0}\right)$ to $\mathrm{A}^{\mathrm{s}}(\mathbb{Z}[G])$ is injective on $\mathrm{R}(\mathbb{Z}[G])$. Thus in the sequel we may view $\mathrm{R}(\mathbb{Z}[G])$ as a subgroup of $\mathrm{A}^{\mathrm{s}}(\mathbb{Z}[G])$.

Viewing $\mathrm{A}^{\mathrm{s}}(\mathbb{Z}[G])$ as a subgroup of $\mathrm{H}^{\mathrm{s}}(\mathbb{Z}[G])$, we obtain the natural decomposition

$$
\mathrm{H}^{\mathrm{s}}(\mathbb{Z}[G])=\mathrm{A}^{\mathrm{s}}(\mathbb{Z}[G]) \times \mathrm{S}_{\infty}(\mathbb{Z}[G]) .
$$

\subsection{Formation of Euler characteristics}

Hermitian Euler characteristics. From now on we fix a set of symplectic $\mathbb{C}[G]$-representations $W_{m}$ whose characters $\theta_{m}$ form a $\mathbb{Z}$-basis of $R_{G}^{\mathrm{s}}$. There is of course a natural $\mathbb{Z}$-basis for $R_{G}^{\mathrm{s}}$ given by the irreducible symplectic characters and the sums of the irreducible non-symplectic characters and their contragredients; in the sequel we shall assume our basis to be of this form. We then fix a non-degenerate $G$-invariant alternating form $\kappa_{m}$ on $W_{m}$ and we let $\left\{w_{m n}\right\}$ denote a hyperbolic basis of $W_{m}$ with respect to $\kappa_{m}$.

Suppose now that we are given a perfect $\mathbb{Z}[G]$-complex $P^{\bullet}$ with $G$-invariant non-degenerate realvalued symmetric forms $\sigma^{\text {ev }}$ (respectively $\sigma^{\text {odd }}$ ) on $\mathrm{H}^{\mathrm{ev}}\left(P_{\mathbb{Q}}^{\bullet}\right)$ (respectively $\mathrm{H}^{\text {odd }}\left(P_{\mathbb{Q}}^{\bullet}\right)$ ). By a result of Swan (see [Ser86, Exercise 16.4]) finitely generated projective $\mathbb{Z}[G]$-modules are locally free. For each prime $p$ of $\mathbb{Z}$ let $\left\{a_{p}^{i j}\right\}_{j}$ denote a $\mathbb{Z}_{p}[G]$-basis for $\mathbb{Z}_{p} \otimes_{\mathbb{Z}} P^{i}$; similarly we choose a $\mathbb{Q}[G]$-basis $\left\{a_{0}^{i j}\right\}_{j}$ for $P_{\mathbb{Q}}^{i}=\mathbb{Q} \otimes_{\mathbb{Z}} P^{i}$; then for each prime $p$ let $\lambda_{p}^{i}$ be the element of $G L\left(\mathbb{Q}_{p}[G]\right)$ such that $\lambda_{p}^{i} a_{p}^{i j}=a_{0}^{i j}$.

As in (2.5) of Definition 2.5, we may construct a $\mathbb{C}$-basis $\left\{b_{j n}^{i m}\right\}_{j n}$ of $\left(W_{m} \otimes_{\mathbb{Q}} P^{i}\right)^{G}$ by letting

$$
b_{j n}^{i m}=r\left(w_{m n} \otimes a_{0}^{i j}\right)=\sum_{g \in G} g w_{m n} \otimes g a_{0}^{i j} .
$$

As previously we shall write $\xi_{m}$ for the canonical isomorphism $\operatorname{det}\left(P_{W_{m}}^{\bullet}\right) \cong \operatorname{det}\left(\mathrm{H}^{\bullet}\left(P_{W_{m}}^{\bullet}\right)\right)$. 


\section{T. Chinburg, G. Pappas and M. Taylor}

Since all the terms in the complexes $P_{\mathbb{Q}}^{\bullet}$ and $\mathrm{H}^{\bullet}\left(P_{\mathbb{Q}}^{\bullet}\right)$ are $\mathbb{Q}[G]$-modules, because the representation $W_{m}$ is symplectic, by Lemma 2.9 it follows that all the terms in the complexes $\left(W_{m} \otimes_{\mathbb{Q}} P_{\mathbb{Q}}^{\bullet}\right)^{G}$ and $\left(W_{m} \otimes_{\mathbb{Q}} \mathrm{H}^{\bullet}\left(P_{\mathbb{Q}}^{\bullet}\right)\right)^{G}$ are even-dimensional. In particular, the remarks in $\S 2.1$ imply that the isomorphism

$$
v_{\mathrm{H}_{m}}: \operatorname{det}\left(\left(W_{m} \otimes_{\mathbb{Q}} \mathrm{H}^{\bullet}\left(P_{\mathbb{Q}}^{\bullet}\right)\right)^{G}\right) \rightarrow \operatorname{det}\left(\left(W_{m} \otimes_{\mathbb{Q}} \mathrm{H}^{\mathrm{ev}}\left(P_{\mathbb{Q}}^{\bullet}\right)\right)^{G}\right) \otimes \operatorname{det}\left(\left(W_{m} \otimes_{\mathbb{Q}} \mathrm{H}^{\operatorname{odd}}\left(P_{\mathbb{Q}}^{\bullet}\right)\right)^{G}\right)^{-1}
$$

is the natural identification with no sign changes.

Definition 5.6. Define $\chi_{\mathrm{H}}^{\mathrm{s}}\left(P^{\bullet}, \sigma\right) \in \mathrm{H}^{\mathrm{s}}(\mathbb{Z}[G])$ to be the class represented under $(5.3)$ by the character map which sends the character $\theta_{m}$ to

$$
\prod_{p<\infty} \operatorname{Det}\left(\lambda_{p}^{i}\right)\left(\theta_{m}\right)^{(-1)^{i}} \times \operatorname{Pf}_{\left(\kappa_{m} \otimes \sigma\right)^{G}}\left(\xi_{m}\left(\bigotimes_{i}\left(\bigwedge_{j n} b_{j n}^{i m}\right)^{(-1)^{i}}\right)\right),
$$

where the terms on the right are taken in lexicographic order. Let $\operatorname{sgn} \cdot \operatorname{pf}\left(P^{\bullet}, \sigma\right)$ be the class in $\mathrm{S}_{\infty}(\mathbb{Z}[G])=\operatorname{Hom}\left(R_{G}^{\mathrm{s}}, \pm 1_{\infty}\right)$ which sends $\theta_{m}$ to 1 (respectively $\left.-1_{\infty}\right)$ if $\operatorname{sgn} . p f\left(\theta_{m}, \sigma, P^{\bullet}\right)$ equals 1 (respectively -1 ).

We now wish to show that these classes are independent of all choices. This is true for sgn.pf $\left(P^{\bullet}, \sigma\right)$ by Proposition 2.10 and Corollary 2.11, so we focus on $\chi_{\mathrm{H}}^{\mathrm{s}}\left(P^{\bullet}, \sigma\right)$.

It is clear from (5.1) that if we change basis from the given $\mathbb{Z}_{p}[G]$-basis for $\mathbb{Z}_{p} \otimes P^{i},\left\{a_{p}^{i j}\right\}_{j}$, then we only change the representing character function by an element in $\operatorname{Det}^{\mathrm{s}}\left(\mathbb{Z}_{p}[G]^{\times}\right) \times 1$. Similarly, if we change the given $\mathbb{Q}[G]$-basis for $\mathbb{Q} \otimes P^{i},\left\{a_{0}^{i j}\right\}_{j}$, then we only change the representing character function by an element in $\operatorname{Im}\left(\Delta^{\mathrm{s}}\right)$.

Next we consider the possible dependence on the alternating forms $\kappa_{m}$ and the chosen hyperbolic basis $\left\{w_{m n}\right\}$. Let $\eta_{m}$ be a further non-degenerate $G$-invariant alternating form on $W_{m}$, let $\left\{w_{m n}^{\prime}\right\}$ denote a hyperbolic basis of $W_{m}$ with respect to $\eta_{m}$, and put

$$
b_{j n}^{i m}=r\left(w_{m n}^{\prime} \otimes a_{0}^{i j}\right)=\sum_{g \in G} g w_{m n}^{\prime} \otimes g a_{0}^{i j} .
$$

In order to show that the value in (5.8) does not change, we must show that

$$
\operatorname{Pf}_{\left(\kappa_{m} \otimes \sigma\right)^{G}}\left(\xi_{m}\left(\bigotimes_{i}\left(\bigwedge_{j n} b_{j n}^{i m}\right)^{(-1)^{i}}\right)\right)=\operatorname{Pf}_{\left(\eta_{m} \otimes \sigma\right)^{G}}\left(\xi_{m}\left(\bigotimes_{i}\left(\bigwedge_{j n} b_{j n}^{\prime i m}\right)^{(-1)^{i}}\right)\right) .
$$

This follows from Proposition 2.10(b) since the map $T: U \rightarrow U$ appearing in this proposition does not depend on the choice of $W$ or of an alternating form on $W$.

Arakelov Euler characteristics. Here we briefly recall the construction of the Arakelov Euler characteristic given in [CPT02]. Let $\left\{V_{r}\right\}$ denote the distinct simple two-sided ideals of the complex group algebra $\mathbb{C}[G]$, and let $\nu_{\mathbb{C}}^{(r)}$ denote the hermitian form on $V_{r}$ given by the restriction of the standard non-degenerate $G$-invariant hermitian form $\nu_{\mathbb{C}}: \mathbb{C}[G] \times \mathbb{C}[G] \rightarrow \mathbb{C}$,

$$
\nu_{\mathbb{C}}\left(\sum_{g \in G} l_{g} g, \sum_{h \in G} m_{h} h\right)=|G| \sum_{g \in G} l_{g} \overline{m_{g}},
$$

and we let $\left\{v_{r s}\right\}$ denote an orthonormal basis of $V_{r}$ with respect to $\nu_{\mathbb{C}}^{(r)}$.

We next suppose that we are given a perfect $\mathbb{Z}[G]$-complex $P^{\bullet}$ with metrics $h=\left\{h_{r}\right\}$ on the equivariant determinant of cohomology, i.e. each $h_{r}$ is a metric on the $\operatorname{complex}$ line $\operatorname{det}\left(\left(V_{r} \otimes \mathbb{Q}\right.\right.$ $\left.\left.\mathrm{H}^{\bullet}\left(P^{\bullet}\right)\right)^{G}\right)$. We again let $\left\{a_{p}^{i j}\right\}_{j}$ denote a $\mathbb{Z}_{p}[G]$-basis for $\mathbb{Z}_{p} \otimes P^{i}$ and let $\left\{a_{0}^{i j}\right\}_{j}$ denote a $\mathbb{Q}[G]$-basis 


\section{Pfaffians And Hodge Discriminants}

for $\mathbb{Q} \otimes P^{i}$; as previously, we let $\lambda_{p}^{i}$ be the element of $G L\left(\mathbb{Q}_{p}[G]\right)$ such that $\lambda_{p}^{i} a_{p}^{i j}=a_{0}^{i j}$. Then for each pair $i, r$ we put

$$
c_{j s}^{i r}=r\left(v_{r s} \otimes a_{0}^{i j}\right)=\sum_{g \in G} g v_{r s} \otimes g a_{0}^{i j} .
$$

As before, $\left\{c_{j s}^{i r}\right\}$ is a $\mathbb{C}$-basis of $\left(V_{r} \otimes \mathbb{Q} P^{i}\right)^{G}$.

Definition 5.7. The equivariant Arakelov class $\chi_{\mathrm{A}}\left(P^{\bullet}, h\right) \in \mathrm{A}(\mathbb{Z}[G])$ is defined to be the class represented by the following homomorphism on characters: if $V_{r}$ has character $\chi_{r}(1) \chi_{r}$, then the complex conjugate $\bar{\chi}_{r}$ is sent to the value

$$
\prod_{p<\infty} \operatorname{Det}\left(\lambda_{p}^{i}\right)\left(\bar{\chi}_{r}\right)^{(-1)^{i}} \times h_{r}\left(\xi_{r}\left(\bigotimes_{i}\left(\bigwedge_{j s} c_{j s}^{i r}\right)^{(-1)^{i}}\right)\right)^{1 / \chi_{r}(1)} .
$$

From $[$ CPT02, $\S 3.3]$ we know that the class given by this character map is again independent of choices. The symplectic Arakelov class $\chi_{\mathrm{A}}^{\mathrm{s}}\left(P^{\bullet}, h\right) \in \mathrm{A}^{\mathrm{s}}(\mathbb{Z}[G])$ is then given by restricting the above character map to symplectic characters.

The hermitian metrics associated to a symmetric bilinear form. With the notation of $\S 5.3$, we suppose that we are given non-degenerate $G$-invariant real-valued symmetric bilinear forms $\sigma^{\text {ev }}, \sigma^{\text {odd }}$ on $\mathrm{H}^{\text {ev }}\left(P_{\mathbb{Q}}^{\bullet}\right), \mathrm{H}^{\text {odd }}\left(P_{\mathbb{Q}}^{\bullet}\right)$. We now briefly recall how these naturally determine a system of metrics on the equivariant determinant of cohomology of $P^{\bullet}$. We observe that $\left(\nu_{\mathbb{C}}^{(r)} \otimes \sigma^{\mathrm{ev}}\right)^{G}$ is a non-degenerate hermitian form on the vector space $\left(V_{r} \otimes_{\mathbb{Q}} \mathrm{H}^{\mathrm{ev}}\left(P_{\mathbb{Q}}^{\bullet}\right)\right)^{G}$; the determinant of this form affords a hermitian form $\operatorname{det}\left(\left(\nu_{\mathbb{C}}^{(r)} \otimes \sigma^{\mathrm{ev}}\right)^{G}\right)$ on the complex line $\operatorname{det}\left(\left(V_{r} \otimes_{\mathbb{Q}} \mathrm{H}^{\mathrm{ev}}\left(P_{\mathbb{Q}}^{\bullet}\right)\right)^{G}\right)$ which may be either positive or negative definite; multiplying by -1 if the form is negative definite, in all cases we then obtain a positive definite form which we denote by $\left|\operatorname{det}\left(\left(\nu_{\mathbb{C}}^{(r)} \otimes \sigma^{\mathrm{ev}}\right)^{G}\right)\right|$. The positive definite form $\left|\operatorname{det}\left(\left(\nu_{\mathbb{C}}^{(r)} \otimes \sigma^{\text {odd }}\right)^{G}\right)\right|$ is defined similarly. We then write $h_{r}$ for the metric on the complex line $\operatorname{det}\left(V_{r} \otimes_{\mathbb{Q}}\left(\mathrm{H}^{\bullet}\left(P_{\mathbb{Q}}^{\bullet}\right)\right)^{G}\right)$ corresponding via $v_{\mathrm{H}^{\bullet}(P \bullet)}$ to the positive definite form

$$
\left|\operatorname{det}\left(\left(\nu_{\mathbb{C}}^{(r)} \otimes \sigma^{\mathrm{ev}}\right)^{G}\right)\right| \otimes\left|\operatorname{det}\left(\left(\nu_{\mathbb{C}}^{(r)} \otimes \sigma^{\text {odd }}\right)^{G}\right)\right|^{-1} .
$$

Then $h=\left\{h_{r}\right\}$ is the required system of metrics on the equivariant determinant of cohomology of $P^{\bullet}$.

Using the method of proof of Proposition 2.10 we obtain the following result. Suppose that $U$ is a free $\mathbb{R}[G]$-module which supports a $G$-invariant symmetric form $\sigma$. Let $V$ be a left ideal of $\mathbb{C}[G]$ endowed with the non-degenerate $G$-invariant hermitian form $\nu_{V}$ given by the restriction of $\nu_{\mathbb{C}}$. Let $T: U \rightarrow U$ be a $\mathbb{C}[G]$-module isomorphism for which (2.11) holds relative to some choice of basis $\left\{u_{i}\right\}_{i=1}^{q}$ for $U$ as a $\mathbb{C}[G]$-module. Let $h_{V}$ denote the hermitian form on the complex vector space $\left(V \otimes_{\mathbb{R}} U\right)^{G}$ given by restricting $(1 / \# G) \nu_{V} \otimes \sigma$. Make $V$ a right $\mathbb{C}[G]$-module via the rule $v g=g^{-1} v$ for $v \in V$ and $g \in G$. The choice of $\left\{u_{i}\right\}_{i}$ then gives an isomorphism $V \otimes_{\mathbb{C}[G]} U \cong V^{q}$; let $T_{V}^{(q)}$ denote the automorphism $1 \otimes_{\mathbb{C}[G]} T$ of this space.

Proposition 5.8. Let $\left\{v_{V, s}\right\}_{s}$ be an orthonormal basis of $V$ with respect to $\nu_{V}$. Then $T_{V}^{(q)}$ is a self-adjoint with respect to the form $\nu_{V}^{(q)}$ on $V^{q}$ which is the direct sum of q copies of $\nu_{V}$. We have

$$
h_{V}\left(\bigwedge_{s, i} r\left(v_{V, s} \otimes u_{i}\right)\right)=\left|\operatorname{det}\left(T_{V}^{(q)}\right)\right|^{1 / 2},
$$

where $r: V^{q}=V \otimes_{\mathbb{C}[G]} U \rightarrow\left(V \otimes_{\mathbb{R}} U\right)^{G}$ sends $v \otimes_{\mathbb{C}[G]} u$ to $\sum_{g \in G} g v \otimes g u$.

Independence of Arakelov classes under quasi-isomorphism. We first recall the following result from [CPT02, Theorem 3.9]. Suppose $P_{1}^{\bullet}$ (respectively $P_{2}^{\bullet}$ ) is a perfect $\mathbb{Z}[G]$-complex which supports 


\section{T. Chinburg, G. Pappas and M. Taylor}

metrics $h^{1}=\left\{h_{r}^{1}\right\}_{r}$ (respectively $h^{2}=\left\{h_{r}^{2}\right\}_{r}$ ) on its equivariant determinant of cohomology. Suppose further that there is a quasi-isomorphism $\phi: P_{1}^{\bullet} \rightarrow P_{2}^{\bullet}$ in the derived category of bounded complexes of finitely generated $\mathbb{Z}[G]$-modules, which has the property that $\phi_{*} h^{1}=h^{2}$. Then we know that the formation of Arakelov classes is natural with respect to quasi-isomorphisms in the sense that $\chi_{\mathrm{A}}\left(P_{1}^{\bullet}, h^{1}\right)=\chi_{\mathrm{A}}\left(P_{2}^{\bullet}, h^{2}\right)$.

Comparison of Euler characteristics. Suppose $W$ is either an irreducible symplectic representation of $G$ or the sum $W_{1} \oplus \bar{W}_{1}$ of an irreducible non-symplectic representation $W_{1}$ of $G$ with its dual. Define $V$ to be the two-sided ideal of $\mathbb{C}[G]$ associated to $W$ in the first case, and the sum of the two-sided ideals $V_{1}$ and $\bar{V}_{1}$ associated to $W_{1}$ and to $\bar{W}_{1}$ in the second case. As a representation of $G, V$ is then isomorphic to the direct sum of $d$ copies of $W$, where $d=\operatorname{dim}_{\mathbb{C}}(W)$ in the first case and $d=\operatorname{dim}_{\mathbb{C}}\left(W_{1}\right)$ in the second case. Comparing the archimedean terms in Definitions 5.6 and 5.7, and using Corollary 2.11, Proposition 5.8 and (2.4) of Proposition 2.1, we see that, under the decomposition $(5.6)$ of $\S 5.2$,

$$
\chi_{\mathrm{H}}^{\mathrm{s}}\left(P^{\bullet}, \sigma\right)=\chi_{\mathrm{A}}^{\mathrm{s}}\left(P^{\bullet}, h_{\sigma}\right) \times \operatorname{sgn} \cdot \operatorname{pf}\left(P^{\bullet}, \sigma\right) .
$$

Since sgn.pf $\left(P^{\bullet}, \sigma\right)$ depends only on the quasi-isomorphism class of $P^{\bullet}$ by the results of $\S 2.3$, we conclude that the same is true of $\chi_{\mathrm{H}}^{\mathrm{s}}\left(P^{\bullet}, \sigma\right)$.

\section{Hodge-Arakelov discriminants}

Throughout this section we make the following assumptions. Let $\mathcal{X}$ be a flat projective scheme over $\operatorname{Spec}(\mathbb{Z})$ which is equidimensional of dimension $d+1$ and which supports the action of a finite group $G$; we let $\mathcal{Y}$ denote the quotient $\mathcal{X} / G$, and we further assume that the following two conditions are satisfied.

(T1) The action of $G$ on $\mathcal{X}$ is 'tame' (for every point $x$ of $\mathcal{X}$ the order of the inertia group $I_{x} \subset G$ is prime to the residual characteristic of $x)$. Since $\mathcal{X}$ maps onto $\operatorname{Spec}(\mathbb{Z})$, it follows that the locus of ramification of the action of $G$ is fibral. We write $X$ (respectively $Y$ ) for the generic fiber $\mathcal{X} \times_{\operatorname{Spec}(\mathbb{Z})} \operatorname{Spec}(\mathbb{Q})\left(\right.$ respectively $\left.\mathcal{Y} \times_{\operatorname{Spec}(\mathbb{Z})} \operatorname{Spec}(\mathbb{Q})\right)$. The group $G$ acts freely on $X$ and so the cover $X \rightarrow Y$ is étale.

(T2) Both schemes $\mathcal{X}$ and $\mathcal{Y}$ are regular and 'tame' (i.e. they are regular and all their special fibers are divisors with normal crossings with multiplicities prime to the residue characteristic).

Let $\Omega_{\mathcal{X} / \mathbb{Z}}^{1}$ denote the coherent sheaf of differentials of $\mathcal{X} \rightarrow \operatorname{Spec}(\mathbb{Z})$. Since $\mathcal{X}$ is regular, we may choose a resolution of $\Omega_{\mathcal{X} / \mathbb{Z}}^{1}$ by a length 2 complex $K^{\bullet}$ of $G$-equivariant locally free $\mathcal{O}_{\mathcal{X}}$ sheaves. For $i \geqslant 0$ we let $L \wedge^{i}$ denote the $i$ th left derived exterior power functor of Dold and Puppe on perfect complexes of $G$-equivariant $\mathcal{O}_{\mathcal{X}}$-sheaves (that is to say, $\mathcal{O}_{\mathcal{X}}$-sheaves with a $G$-action which is compatible with the $G$-action on $\mathcal{O}_{\mathcal{X}}$ ). Thus $L \wedge^{i} K^{\bullet}$ denotes the complex arising from the application of $L \wedge^{i}$ to $K^{\bullet}$ and we define $L \wedge^{\bullet} \Omega_{\mathcal{X} / \mathbb{Z}}^{1}$ to be the direct sum of the complexes $L \wedge^{i}$ $K^{\bullet}[-i]$ for $0 \leqslant i \leqslant d$. For details of the Dold-Puppe exterior power functor, the reader is referred to [DP61], [Ill71] and [SABK92, §§ 5.4-5.9].

We recall from [CEPT96] that, because $G$ acts tamely, $R \Gamma\left(\mathcal{X}, L \wedge^{\bullet} \Omega_{\mathcal{X} / \mathbb{Z}}^{1}\right)$ may represented by a perfect $\mathbb{Z}[G]$-complex. Note for future reference that on the generic fiber $X$ of $\mathcal{X}$ each $\left(L \wedge^{i} K^{\bullet}\right) \otimes_{\mathbb{Z}} \mathbb{Q}$ is quasi-isomorphic to the sheaf of differentials $\Omega_{X / \mathbb{Q}}^{i}$ viewed as a complex concentrated in degree 0 .

We begin by considering the hermitian Euler characteristics associated to $R \Gamma\left(\mathcal{X}, L \wedge \bullet \Omega_{\mathcal{X} / \mathbb{Z}}^{1}\right)$ when this complex is endowed with the duality pairings described in $\S 3.1$. Next we consider various Arakelov Euler characteristics associated to the de Rham cohomology of $\mathcal{X}$; we then conclude by piecing this all together to prove Theorem 1.3. 


\section{Pfaffians AND Hodge DiscRiminants}

\subsection{Hodge Euler characteristics}

Let $\sigma=\left\{\sigma^{\text {ev }}, \sigma^{\text {odd }}\right\}$ denote the $G$-invariant forms on the Hodge cohomology $\mathrm{H}_{\text {Hod }}^{t}(X / \mathbb{Q})[d]$ of $L \wedge \bullet \Omega_{X / \mathbb{Q}}^{1}$ considered in $\S 3.1$. Because hypothesis (T1) is satisfied, we know from [CEPT96] that the complex $R \Gamma\left(\mathcal{X}, L \wedge^{\bullet} \Omega_{\mathcal{X} / \mathbb{Z}}^{1}\right)$ is represented by a perfect $\mathbb{Z}[G]$-complex $P^{\bullet}$. We let $\sigma_{P} \bullet$ denote the induced forms on the cohomology of $P^{\bullet}$, and we set

$$
\chi_{\mathrm{H}}^{\mathrm{s}}\left(R \Gamma\left(\mathcal{X}, L \wedge^{\bullet} \Omega_{\mathcal{X} / \mathbb{Z}}^{1}\right), \sigma\right):=\chi_{\mathrm{H}}^{\mathrm{s}}\left(P^{\bullet}, \sigma_{P} \bullet\right) .
$$

From $\S 5.3$ we know that the formation of hermitian Euler characteristics is invariant under quasiisomorphism and so indeed the above hermitian Euler characteristic is independent of the complex $P^{\bullet}$ chosen.

Writing $h_{\sigma}$ for the hermitian metrics on the determinant of cohomology associated to $\sigma$, from (5.9) we know that we can write

$$
\chi_{\mathrm{H}}^{\mathrm{s}}\left(R \Gamma\left(\mathcal{X}, L \wedge^{\bullet} \Omega_{\mathcal{X} / \mathbb{Z}}^{1}\right), \sigma\right)=\chi_{A}^{\mathrm{s}}\left(R \Gamma\left(\mathcal{X}, L \wedge^{\bullet} \Omega_{\mathcal{X} / \mathbb{Z}}^{1}\right), h_{\sigma}\right) \times \operatorname{sgn} \cdot \operatorname{pf}\left(R \Gamma\left(L \wedge^{\bullet} \Omega_{X / \mathbb{Q}}^{1}\right), \sigma\right) .
$$

Furthermore, by Theorem 1.1, we know that the sgn.pf term is completely determined by the archimedean $\varepsilon$-constants of $\mathcal{X}$. Therefore, in order to describe fully the hermitian Hodge Euler characteristic $\chi_{\mathrm{H}}^{\mathrm{s}}\left(R \Gamma\left(\mathcal{X}, L \wedge^{\bullet} \Omega_{\mathcal{X} / \mathbb{Z}}^{1}\right), \sigma\right)$, we now need to describe the Arakelov Euler characteristic $\chi_{A}^{\mathrm{s}}\left(R \Gamma\left(\mathcal{X}, L \wedge^{\bullet} \Omega_{\mathcal{X} / \mathbb{Z}}^{1}\right), h_{\sigma}\right)$. We shall now relate this Arakelov Euler characteristic to those studied in $[\mathrm{CPT} 02]$.

\section{$6.2 L^{2}$-norms}

In this section we consider the $L^{2}$-norms on the Hodge cohomology groups of $X$.

Given a Kähler metric $h_{Y}$ on the complex tangent space $T Y$ of an arithmetic variety $\mathcal{Y}$, which is invariant under complex conjugation, we denote by $h_{X}=h^{T X}$ the Kähler metric on $X(\mathbb{C})$ given by the pullback of $h_{Y}$; this then is also invariant under complex conjugation. Define $h_{X}^{D}$ to be the metric on the complex cotangent space of $X(\mathbb{C})$ which is dual to $h_{X}$.

Let $d_{X}$ denote the volume form given by the $d$ th exterior power of the $(1,1)$-form associated to $h_{X}^{D}$. Define the $L^{2}$ inner product on the smooth forms

$$
\mathcal{A}^{0, q}\left(X(\mathbb{C}), \Omega_{X(\mathbb{C})}^{p}\right)=\mathcal{A}^{0, q}(X) \otimes_{C^{\infty}(X(\mathbb{C}))} \mathcal{A}^{p, 0}(X)=\mathcal{A}^{p, q}(X(\mathbb{C}))
$$

by

$$
\langle s, t\rangle_{X}=\frac{1}{|G| d !} \int_{X(\mathbb{C})} \wedge^{p+q} h_{X}^{D}(s(x), t(x))\left(\frac{i}{2 \pi}\right)^{d} d_{X},
$$

where $\wedge^{p+q} h_{X}^{D}(-,-)$ denotes the inner product on $p+q$ forms given by the $(p+q)$ th exterior product of $h_{X}^{D}$ (see for instance [SABK92, $\S$ V.2.2 and p. 131]). The reason for the normalization factor $(i / 2 \pi)^{d}$ on the volume form will become apparent below: it will ensure that the corresponding $L^{2}$-norm is compatible with Serre duality pairings of $\S 3.1$. The reason for normalizing by the factor $|G|^{-1}$ is that, since $X \rightarrow Y$ is étale, our metrics are then natural with respect to pullback in the sense that, for $p$-forms $s^{\prime}, t^{\prime}$ on $Y$, we then have $\left\langle\pi^{*} s^{\prime}, \pi^{*} t^{\prime}\right\rangle_{X}=\left\langle s^{\prime}, t^{\prime}\right\rangle_{Y}$ where

$$
\left\langle s^{\prime}, t^{\prime}\right\rangle_{Y}=\frac{1}{d !} \int_{Y(\mathbb{C})} \wedge^{p+q} h_{Y}^{D}\left(s^{\prime}(y), t^{\prime}(y)\right)\left(\frac{i}{2 \pi}\right)^{d} d_{Y}
$$

and $d_{Y}$ is the volume form given by the $d$ th exterior power of the $(1,1)$-form associated to $h_{Y}^{D}$. Let $\Delta^{q}=\overline{\partial \partial}^{*}+\bar{\partial}^{*} \bar{\partial}$ be the Laplace operator on $\mathcal{A}^{p, q}(X(\mathbb{C}))$. The Hodge isomorphism

$$
\mathrm{H}^{q}\left(X(\mathbb{C}), \Omega_{X(\mathbb{C})}^{p}\right)=\operatorname{ker}\left(\Delta^{q}\right)
$$

then gives an $L^{2}$-norm on $\mathrm{H}^{q}\left(X(\mathbb{C}), \Omega_{X(\mathbb{C})}^{p}\right)$. 


\section{T. Chinburg, G. Pappas and M. Taylor}

Let $\widehat{G}$ denote the set of complex irreducible characters of $G$ and let $\phi \in \widehat{G}$. Recall that the set of one-dimensional $\mathbb{C}$-vector spaces given by the determinants of the different $\phi$-isotypic subspaces of cohomology is called the equivariant determinant of cohomology. (Thus, in the terminology of $[\mathrm{B}], \log |\cdot|_{L^{2}, \phi}$ is the coefficient of $\phi$ in the symbol $\log |\cdot|_{L^{2}}$.) We let $|G|^{-1}|\cdot|_{L^{2}, \phi}$ denote the induced metric on the determinant of the $\phi$-isotypic part of the cohomology of $\Omega_{X(\mathbb{C})}^{\bullet}$, and we denote the resulting $L^{2}$-metric on the equivariant determinant of cohomology of $\Omega_{X(\mathbb{C})}^{\bullet}$ by $|G|^{-1} \wedge^{\bullet}|\cdot|_{L^{2}}$ in order to emphasize the appearance of the scaling factor $|G|^{-1}$.

Identifying $\mathrm{H}^{d}\left(X(\mathbb{C}), \Omega_{X(\mathbb{C})}^{d}\right)$ with the Dolbeault cohomology group $\mathrm{H}_{\bar{\partial}}^{d, d}(X)$ and then integrating over $X$ affords a surjection

$$
\mathrm{H}^{d}\left(X, \Omega_{X}^{d}\right) \otimes \mathbb{C}=\mathrm{H}^{d}\left(X(\mathbb{C}), \Omega_{X(\mathbb{C})}^{d}\right) \stackrel{\int_{X}}{\longrightarrow} \mathbb{C} .
$$

From the above discussion we know that the following diagram commutes.

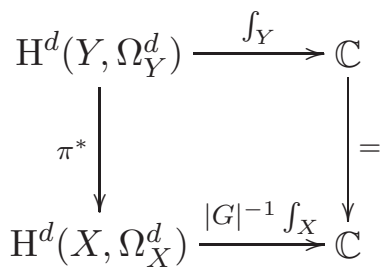

We then define the trace map

$$
\mathrm{H}^{d}\left(X, \Omega_{X}^{d}\right) \stackrel{|G|^{-1} T r}{\longrightarrow} \mathbb{Q}
$$

to be induced by the map

$$
\frac{i^{d}}{(2 \pi)^{d} d !|G|} \int_{X}
$$

Recall that, by Proposition 3.4, the following diagram commutes:

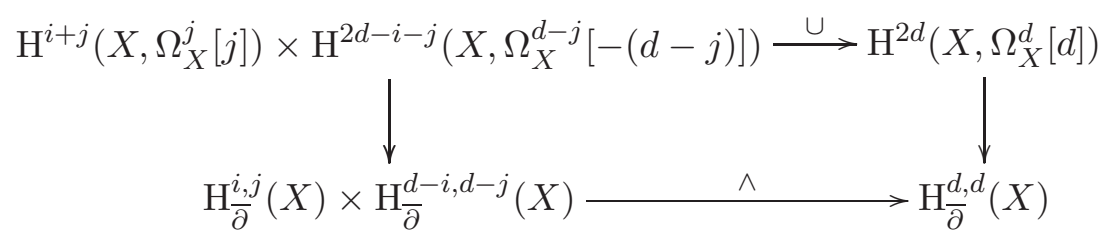

where the upper horizontal map is the cup-product, the lower horizontal map is the exterior product of differential forms, and the vertical arrows are Dolbeault isomorphisms. The $L^{2}$-norms $|G|^{-1}|\cdot|_{L^{2}}$ we have constructed on the Dolbeault cohomology groups in the bottom row of (6.4) are compatible with the cap-product pairing on forms and with Serre duality (see [GSZ91, § 1.4] and also the proof of Theorem 7.8 in [CPT02]). By grouping together terms corresponding to forms of type $(i, j)$ with forms of type $(d-i, d-j)$, we see from (6.4) that the metrics $h_{\sigma}=\left\{h_{r}\right\}$ induced by $\sigma$ on the $\mathbb{C}$-lines which form the equivariant determinant of cohomology coincide with the metrics induced by $|G|^{-1}|\cdot|_{L^{2}}$. In summary we have now shown the following result.

Lemma 6.1. The metrics $h_{\sigma}=\left\{h_{r}\right\}$, associated to the Hodge pairings $\sigma_{i, j}$ of $\S 3.1$, coincide with the metric $|G|^{-1} \wedge \cdot|\cdot|_{L^{2}}$ on the equivariant determinant of cohomology of $\Omega_{X(\mathbb{C})}^{\bullet}$. Therefore

$$
\chi_{\mathrm{A}}\left(R \Gamma\left(\mathcal{X}, L \wedge \bullet \Omega_{\mathcal{X} / \mathbb{Z}}^{1}\right)[d], h_{\sigma}\right)=\chi_{\mathrm{A}}\left(R \Gamma\left(\mathcal{X}, L \wedge^{\bullet} \Omega_{\mathcal{X} / \mathbb{Z}}^{1}\right)[d],|G|^{-1} \wedge^{\bullet}|\cdot|_{L^{2}}\right)
$$

and the same equality holds if $\chi_{\mathrm{A}}$ is replaced on both sides by $\chi_{\mathrm{A}}^{\mathrm{s}}$. 


\section{PfaffiAns AND HodGe DISCRIMINANTS}

\subsection{Proof of Theorem 1.3}

We denote by $|G|^{-1} \wedge h_{X, Q}^{D}$, the Quillen metric on the equivariant determinant of cohomology of $\Omega_{X(\mathbb{C})}^{p}$ associated to $|G|^{-1} \wedge^{p} h_{X}^{D}$. Recall that this is constructed by multiplying by the inverse of the equivariant analytic torsion associated to $|G|^{-1} \wedge^{p} h_{X}^{D}$, which we will denote $T_{\phi}\left(\Omega_{X(\mathbb{C})}^{p},|G|^{-1} \wedge^{p} h_{X}^{D}\right)$; see [Bis95] for full details of this construction.

By (6.2) and (6.5), in order to prove Theorem 1.3, it will suffice to show the following theorem.

TheOREM 6.2. One has

$$
\prod_{p=0}^{d} T_{\phi}\left(\Omega_{X(\mathbb{C})}^{p},|G|^{-1} \wedge^{p} h_{X}^{D}\right)^{(-1)^{p}}=1
$$

and so

$$
\chi_{\mathrm{A}}\left(R \Gamma\left(\mathcal{X}, L \wedge^{\bullet} \Omega_{\mathcal{X} / \mathbb{Z}}^{1}\right)[d],|G|^{-1} \wedge^{\bullet}|\cdot|_{L^{2}}\right)=\chi_{\mathrm{A}}\left(R \Gamma\left(\mathcal{X}, L \wedge^{\bullet} \Omega_{\mathcal{X} / \mathbb{Z}}^{1}\right)[d],|G|^{-1} \wedge^{\bullet} h_{X, Q}^{D}\right) .
$$

Proof. The proof proceeds in two steps. Firstly we show that

$$
\prod_{p=0}^{d} T_{\phi}\left(\Omega_{X(\mathbb{C})}^{p}, \wedge^{p} h_{X}^{D}\right)^{(-1)^{p}}=1 .
$$

The proof of this is the same as that of Theorem 3.1 in [RS73] mutatis mutandis; see [MR04].

Secondly, in order to deduce (6.6) from (6.8), note that $|G|^{-1} \wedge \cdot|\cdot|_{L^{2}}$ arises from the set of metrics $|G|^{-1}|\cdot|_{L^{2}}$ coming from scaling by $|G|^{-1}$ (on forms of all weights) the metrics $|\cdot|_{L^{2}}$. The associated Laplacians remain unchanged, and so their analytic torsions coincide, that is to say

$$
T_{\phi}\left(\Omega_{X(\mathbb{C})}^{p},|G|^{-1} \wedge^{p} h_{X}^{D}\right)=T_{\phi}\left(\Omega_{X(\mathbb{C})}^{p}, \wedge^{p} h_{X}^{D}\right)
$$

as required.

\section{Non-archimedean invariants}

In this section we fix a prime number $p$ and we adopt the notation of the previous section but with the base $\operatorname{Spec}(\mathbb{Z})$ replaced by $\operatorname{Spec}\left(\mathbb{Z}_{p}\right)$. Thus $\mathcal{X}$ is again a regular scheme but which is now projective and flat over $\operatorname{Spec}\left(\mathbb{Z}_{p}\right)$ and which satisfies the hypotheses $(\mathrm{T} 1)$ and $(\mathrm{T} 2)$ stated in $\S 6$ when $\mathbb{Z}$ is replaced by $\mathbb{Z}_{p}$. The principal goal of this section is to build on the work in [CEPT98] in order to produce a Pfaffian characterization of non-archimedean local $\varepsilon$-constants.

\subsection{Characterization of $\varepsilon_{0}$}

7.1.1 The Pfaffian divisor. We begin by recalling a number of results from [CEPT98]. Let $\left\{b_{i}\right\}_{i} \in I$ denote the distinct irreducible components of the reduced special fiber $\mathcal{Y}_{p}^{\text {red }}$ of $\mathcal{Y}$. For each $i \in I$ we choose an irreducible component $B_{i}$ of $\mathcal{X}_{p}^{\text {red }}$ above $b_{i}$; we let $I_{i}$ denote the inertia group of the generic point of $B_{i}$ and we let $u_{i}$ denote the augmentation character of $I_{i}$, that is to say the regular character of $I_{i}$ minus the trivial character. Let $\psi$ be a symplectic character of $G$ with values in $\overline{\mathbb{Q}}$. Since the character $\operatorname{Ind}_{I_{i}}^{G} u_{i}$ takes integer values, we know by Lemma 2.9 that the value of the character inner product $\left(\operatorname{Ind}_{I_{i}}^{G} u_{i}, \psi\right)$ is necessarily an even integer. As a result, this inner product does not change if we view $\psi$ as a symplectic character with values in $\overline{\mathbb{Q}}_{p}$ via some choice of an embedding of $\overline{\mathbb{Q}}$ into $\overline{\mathbb{Q}}_{p}$. (Note that in $\S 2$ (respectively $\S 3$ ) of [CEPT98], $\psi$ is considered to have values in $\overline{\mathbb{Q}}_{p}$ (respectively $\overline{\mathbb{Q}}$ ).) We define the Pfaffian divisor associated to $\psi$ to be the $\mathcal{Y}$-divisor, supported on the special fiber, given by

$$
\operatorname{Pf}(\mathcal{Y}, \psi)=\sum_{i} \frac{1}{2}\left(\operatorname{Ind}_{I_{i}}^{G} u_{i}, \psi\right) b_{i}
$$




\section{T. Chinburg, G. Pappas and M. Taylor}

Let $\Omega_{\mathcal{Y} / \mathbb{Z}_{p}}^{1}\left(\log \mathcal{Y}_{p}^{\text {red }} / \log \mathbb{F}_{p}\right)$ denote the sheaf of $\mathcal{Y}$-differentials with at worst logarithmic singularities along the special fiber $\mathcal{Y}_{p}$ of $\mathcal{Y}$ (see for instance $[\mathrm{K}]$ for full details). We recall that, with our hypothe-

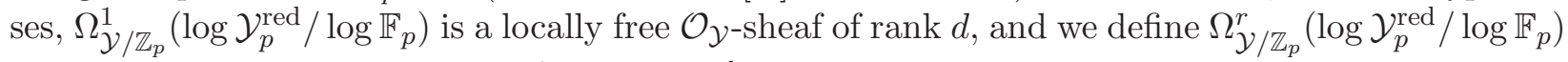
to be the $r$ th exterior power of $\Omega_{\mathcal{Y} / \mathbb{Z}_{p}}^{1}\left(\log \mathcal{Y}_{p}^{\text {red }} / \log \mathbb{F}_{p}\right)$. With the usual $\lambda$-ring notation we put

$$
\lambda_{-1}\left(\Omega_{\mathcal{Y} / \mathbb{Z}_{p}}^{1}\left(\log \mathcal{Y}_{p}^{\text {red }} / \log \mathbb{F}_{p}\right)\right)=\sum_{r=0}^{d}(-1)^{r} \Omega_{\mathcal{Y} / \mathbb{Z}_{p}}^{r}\left(\log \mathcal{Y}_{p}^{\text {red }} / \log \mathbb{F}_{p}\right)
$$

in the Grothendieck group $K_{0}(Y)$ of locally free sheaves on $Y$. In [CEPT98, §3] we defined a character function $k^{\prime} \in \operatorname{Hom}_{\Omega}\left(R_{G}^{\mathrm{s}}, \mathbb{Z}\right)$ by the rule that

$$
k^{\prime}(\psi)=\operatorname{deg}\left(\lambda_{-1}\left(\Omega_{\mathcal{Y} / \mathbb{Z}_{p}}^{1}\left(\log \mathcal{Y}_{p}^{\text {red }} / \log \mathbb{F}_{p}\right)\right) \cdot \operatorname{Pf}(\mathcal{Y}, \psi)\right),
$$

where the right-hand side has the following meaning. For an integral fibral divisor $D$ of $\mathcal{Y}$ the support of $\lambda_{-1}\left(\Omega_{\mathcal{Y} / \mathbb{Z}_{p}}^{1}\left(\log \mathcal{Y}_{p}^{\text {red }} / \log \mathbb{F}_{p}\right)\right) \otimes \mathcal{O}_{D}$ is entirely punctual and its degree over $\mathbb{F}_{p}$ is equal to $(-1)^{d}$ times the degree of the top Chern class of $\left.\Omega_{\mathcal{Y} / \mathbb{Z}_{p}}^{1}\left(\log \mathcal{Y}_{p}^{\text {red }} / \log \mathbb{F}_{p}\right)\right|_{D}$. We then extend this definition to all fibral divisors of $\mathcal{Y}$ by linearity.

Definition 7.1. Let $L=\mathbb{Q}\left(\zeta_{p}\right)$. Define $c$ to be the class in the finite adelic hermitian class group $\operatorname{AdHCL}_{f}\left(O_{L}[G]\right)$ of Definition 5.2 represented by the following character function. $k \in$ $\operatorname{Hom}_{\Omega_{L}}\left(R_{G}^{\mathrm{s}}, J_{f}(\bar{L})\right)$. For each symplectic character $\psi$, the semi-local component of the idele $k(\psi) \in$ $J_{f}(\bar{L})$ at the finite place $v$ of $L$ is

$$
k(\psi)_{v}= \begin{cases}(-p)^{k^{\prime}(\psi)} & \text { if } v \mid p, \\ 1 & \text { if } v \nmid p .\end{cases}
$$

Note that by Frobenius reciprocity, $k^{\prime}(\psi)=0$ and $k(\psi)=1$ if $\psi$ is a sum of copies of the trivial character of $G$.

7.1.2 p-adic absolute values. Let $\overline{\mathbb{Q}}_{p}$ be a chosen algebraic closure of $\mathbb{Q}_{p}$, set $\Omega_{p}=\operatorname{Gal}\left(\overline{\mathbb{Q}}_{p} / \mathbb{Q}_{p}\right)$ and let $R_{G, p}$ denote the ring of $\overline{\mathbb{Q}}_{p}$-valued characters of $G$. We fix an embedding $L \hookrightarrow \overline{\mathbb{Q}}_{p}$, we let $L_{p}$ denote the closure of $L$ in $\overline{\mathbb{Q}}_{p}$, and we let $\lambda$ denote a chosen root of $T^{p-1}+p$ in $L_{p}$. Fix a group homomorphism $\mathbb{Q} \rightarrow \overline{\mathbb{Q}}_{p}^{\times}$which sends $1 \in \mathbb{Q}$ to $\lambda$, and let $\lambda^{\mathbb{Q}}$ be the image of this homomorphism. Given $x \in \overline{\mathbb{Q}}_{p}^{\times}$, define $\|x\|_{p} \in \lambda^{\mathbb{Q}}$ by stipulating that $x \cdot\|x\|_{p}$ is a $p$-adic unit. Let $|x|_{p}$ be the value on $x$ of the unique extension to $\overline{\mathbb{Q}}_{p}$ of the usual $p$-adic absolute value on $\mathbb{Q}$. It is important to keep in mind that $\|x\|_{p} \neq|x|_{p}$ in general, even if $x \in \mathbb{Q}$, since $\lambda \mathbb{Q}^{\mathbb{Q}} \mathbb{Q}=(-p)^{\mathbb{Z}}$.

For $g \in \operatorname{Hom}\left(R_{G, p}, \overline{\mathbb{Q}}_{p}^{\times}\right)$, we say that $\|g\|_{p}$ is well defined if $\|g\|_{p}$ takes values in $\lambda^{\mathbb{Z}}$. If $\|g\|_{p}$ is well defined and if $g$ commutes with the action of $\Omega_{L_{p}}=\operatorname{Gal}\left(\overline{\mathbb{Q}}_{p} / L_{p}\right)$, then $\|g\|_{p}$ also commutes with the action of $\Omega_{L_{p}}$.

For brevity we shall write $(\overline{\mathbb{Q}})_{p}$ for $\left(\overline{\mathbb{Q}} \otimes_{\mathbb{Q}} \mathbb{Q}_{p}\right)$ and we extend the embedding $L \hookrightarrow \overline{\mathbb{Q}}_{p}$ to an embedding $h: \overline{\mathbb{Q}} \hookrightarrow \overline{\mathbb{Q}}_{p}$. This then induces an isomorphism (see [Frö83, II.2.1])

$$
h^{*}: \operatorname{Hom}_{\Omega_{L}}\left(R_{G},(\overline{\mathbb{Q}})_{p}^{\times}\right) \rightarrow \operatorname{Hom}_{\Omega_{L_{p}}}\left(R_{G, p}, \overline{\mathbb{Q}}_{p}^{\times}\right) .
$$

For $f \in \operatorname{Hom}_{\Omega}\left(R_{G},(\overline{\mathbb{Q}})_{p}^{\times}\right)$we say that $\|f\|_{p}$ is well defined if and only if $\left\|h^{*}(f)\right\|_{p}$ is well defined and, in that case, we set

$$
\|f\|_{p}=h^{*-1}\left(\left\|h^{*}(f)\right\|_{p}\right) .
$$

7.1.3 The local constant $\varepsilon_{0}$. In this section we briefly recall a number of properties of local constants and the main result of [CEPT98]. 


\section{Pfaffians And Hodge Discriminants}

Let $d x$ be a Haar measure on $\mathbb{Q}_{p}$ and let $\psi$ be a non-trivial additive character of $\mathbb{Q}_{p}$. Let ${ }^{\prime} V=\left(V^{\prime}, N\right)$ be a continuous complex representation of the Weil-Deligne group (see [Del74, $\left.\S 8\right]$ ). Thus $V^{\prime}$ is a continuous complex representation of the Weil group $W_{\mathbb{Q}_{p}}$ and $N$ is a nilpotent endomorphism of $V^{\prime}$. In [Del74, $\S 5.1$ ], Deligne defines the local constant $\varepsilon\left({ }^{\prime} V, d x, \psi\right) \in \mathbb{C}^{*}$. The constant $\varepsilon_{0}\left({ }^{\prime} V, d x, \psi\right)$ is defined by

$$
\varepsilon_{0}\left({ }^{\prime} V, d x, \psi\right)=\operatorname{det}\left(-F \mid V^{\prime I}\right) \varepsilon\left({ }^{\prime} V, d x, \psi\right),
$$

where $V^{\prime I}$ is the subspace fixed by the inertia subgroup $I$ of $W_{\mathbb{Q}_{p}}$ and $F \in W_{\mathbb{Q}_{p}}$ induces the inverse of the Frobenius automorphism on residue class fields.

The function ' $V \rightarrow \varepsilon_{0}\left({ }^{\prime} V, d x, \psi\right)$ extends to virtual representations of the Weil-Deligne group by linearity. By the standard transformation formula (see [Del74, $\S 5.1]$ ), $\varepsilon_{0}\left({ }^{\prime} V, d x, \psi\right)$ is independent of the choices of $d x$ and $\psi$ if $V^{\prime}$ is of dimension 0 and has trivial determinant; in this case we write $\varepsilon_{0}\left({ }^{\prime} V\right)$ for $\varepsilon_{0}\left({ }^{\prime} V, d x, \psi\right)$.

Once and for all we choose a prime number $l$ different from $p$ and an embedding $j: \mathbb{Q}_{l} \rightarrow \mathbb{C}$. For each $i, 0 \leqslant i \leqslant 2 d$, we consider the étale cohomology group $\mathrm{H}_{l}^{i}=\mathrm{H}_{\text {et }}^{i}\left(\mathcal{X} \times \overline{\mathbb{Q}}_{p}, \mathbb{Q}_{l}\right)$. By following the procedure in $\left[\right.$ Del74, $\S 8$, each $j_{*} \mathrm{H}_{l}^{i}$ affords an open kernel representation of the Weil-Deligne group ${ }^{\prime} W_{\mathbb{Q}_{p}}$. Since the action of $G$ is defined over $\mathbb{Q}$, these representations extend to representations of ${ }^{\prime} W_{\mathbb{Q}_{p}} \times G$ (see [CEPT97, § 2] for details). By [CEPT97, Proposition 2.4.1] the constant $\varepsilon_{0}\left(\left(j_{*} \mathrm{H}_{l}^{i} \otimes\right.\right.$ $\left.V)^{G}\right)$ is independent of the choices of Haar measure and additive character for a complex symplectic representation $V$ of $G$ dimension 0 . We now regard $l$ and $j$ as fixed and define

$$
\varepsilon_{0}\left(\mathcal{X} \otimes_{G} V\right)=\prod_{i=0}^{2 d} \varepsilon_{0}\left(\left(j_{*} \mathrm{H}_{l}^{i} \otimes V\right)^{G}\right)^{(-1)^{i}} .
$$

For an arbitrary representation $V$ of $G$ we set

$$
\tilde{\varepsilon}_{0}\left(\mathcal{X} \otimes_{G} V\right)=\varepsilon_{0}\left(\mathcal{X} \otimes_{G}(V-\operatorname{dim}(V) .1)\right)
$$

and we write $\tilde{\varepsilon}_{0}(\mathcal{X})$ for the resulting function $\chi_{V} \rightarrow \tilde{\varepsilon}_{0}\left(\mathcal{X} \otimes_{G} V\right)$ on $R_{G}$. Let $\tilde{\varepsilon}_{0}^{\mathrm{s}}(\mathcal{X})$ be the restriction of $\tilde{\varepsilon}_{0}(\mathcal{X})$ to $R_{G}^{\mathrm{s}}$.

Proposition 7.2. One has

$$
\tilde{\varepsilon}_{0}^{\mathrm{s}}(\mathcal{X}) \in \operatorname{Hom}_{\Omega_{\mathbb{Q}}}\left(R_{G}^{\mathrm{s}}, \pm p^{\mathbb{Z}}\right) .
$$

Proof. See Propositions 4.2 and 4.3 in [CEPT98].

Proposition 7.3. Let $L=\mathbb{Q}\left(\zeta_{p}\right)$ and suppose that $v$ is a place of $\mathbb{Q}$. Write $L_{v}=L \otimes_{\mathbb{Q}} \mathbb{Q}_{v}$, and let $O_{L_{v}}$ be the integral closure of $1 \otimes \mathbb{Z}_{v}$ in $L_{v}$. Let $\tilde{\varepsilon}_{0}^{\mathrm{s}}(\mathcal{X})_{v}$ be the composition of $\tilde{\varepsilon}_{0}^{\mathrm{s}}(\mathcal{X})$ with the inclusion $\overline{\mathbb{Q}} \rightarrow(\overline{\mathbb{Q}})_{v}^{\times}=\left(\overline{\mathbb{Q}} \otimes_{\mathbb{Q}} \mathbb{Q}_{v}\right)^{\times}$.

(a) One has that $\left\|\tilde{\varepsilon}_{0}(\mathcal{X})\right\|_{p}$ is well defined, and

$$
\left\|\tilde{\varepsilon}_{0}(\mathcal{X})\right\|_{p} . \tilde{\varepsilon}_{0}(\mathcal{X}) \in \operatorname{Det}\left(O_{L_{p}}[G]^{\times}\right) .
$$

(b) For a prime number $l$ different from $p$, one has $\tilde{\varepsilon}_{0}(\mathcal{X})_{l} \in \operatorname{Det}\left(O_{L_{l}}[G]^{\times}\right)$.

(c) Recall that the idele-valued function $k$ on $R_{G}^{\mathrm{s}}$ was defined in (7.2). Then, for $\psi \in R_{G}^{\mathrm{s}}$, one has

$$
\left\|\tilde{\varepsilon}_{0}^{\mathrm{S}}(\mathcal{X})\right\|_{p}(\psi)=k(\psi)_{p}^{-1} .
$$

Proof. Parts (a) and (b) are shown in [CEPT98, Theorem 4]. To show part (c), we know from Proposition 7.2 that $\tilde{\varepsilon}_{0}^{\mathrm{s}}(\mathcal{X})(\psi) \in \pm p^{\mathbb{Z}}$. Therefore

$$
\left\|\tilde{\varepsilon}_{0}^{\mathrm{s}}(\mathcal{X})\right\|_{p}(\psi)=(-p)^{-v_{p}\left(\tilde{\varepsilon}_{0}^{\mathrm{s}}(\mathcal{X})(\psi)\right)}
$$




\section{T. Chinburg, G. Pappas and M. Taylor}

since $\lambda^{p-1}=-p$, where $v_{p}$ is the usual $p$-adic valuation on $\mathbb{Q}^{*}$. The equality in part (c) is now a consequence of the definition of $k(\psi)_{p}$ in $(7.2)$ and the equality

$$
v_{p}\left(\widetilde{\varepsilon}_{0}^{\mathrm{s}}(\mathcal{X})(\psi)\right)=k^{\prime}(\psi)
$$

shown in [CEPT98, Proposition 5.2].

From [CEPT98, Theorem 1] we know the following.

Theorem 7.4. Define the group $R C\left(O_{L}[G]\right)$ of rational classes in $\operatorname{AdHCL}_{f}\left(O_{L}[G]\right)$ to be

$$
R C\left(O_{L}[G]\right)=\frac{\operatorname{Hom}_{\Omega_{\mathbb{Q}}}\left(R_{G}^{s}, \mathbb{Q}^{*}\right) \cdot \operatorname{Det}^{\mathrm{s}}\left(U_{f}\left(O_{L}[G]\right)\right)}{\operatorname{Det}^{\mathrm{S}}\left(U_{f}\left(O_{L}[G]\right)\right)},
$$

where

$$
\operatorname{AdHCL}_{f}\left(O_{L}[G]\right)=\frac{\operatorname{Hom}_{\Omega_{L}}\left(R_{G}^{s}, J_{f}(\bar{L})\right)}{\operatorname{Det}^{\mathrm{s}}\left(U_{f}\left(O_{L}[G]\right)\right)} .
$$

Since $L=\mathbb{Q}\left(\zeta_{p}\right)$, the natural homomorphism $\operatorname{Hom}_{\Omega_{\mathbb{Q}}}\left(R_{G}^{\mathrm{s}}, \mathbb{Q}^{*}\right) \rightarrow R C\left(O_{L}[G]\right)$ is an isomorphism; we let

$$
\theta: R C\left(O_{L}[G]\right) \rightarrow \operatorname{Hom}_{\Omega_{\mathbb{Q}}}\left(R_{G}^{\mathrm{s}}, \mathbb{Q}^{*}\right)
$$

be the inverse of this isomorphism. The class $c$ defined in Definition 7.1 is in the subgroup $R C\left(O_{L}[G]\right)$. If $V$ is a symplectic representation of $G$ with character $\psi$ then

$$
\theta(c)(\psi)=\varepsilon_{0}\left(\mathcal{X} \otimes_{G}(V-\operatorname{dim}(V) \cdot 1)\right) .
$$

\subsection{The non-archimedean invariant $\|\operatorname{Pf}(\mathcal{X})\|_{p}$}

In this section we present the non-archimedean Pfaffian invariant which we require for the characterization of non-archimedean $\varepsilon$-constants.

7.2.1 Duality maps. Recall that $\pi: \mathcal{X} \rightarrow \mathcal{Y}=\mathcal{X} / G$ is the quotient map associated to the $G$-action on $\mathcal{X}$. We let $E_{1}^{\bullet}$ denote the complex of length 2 ,

$$
E_{1}^{\bullet}: \pi_{*} \mathcal{O}_{\mathcal{X}} \stackrel{T r}{\longrightarrow} \operatorname{Hom}_{\mathcal{O}_{\mathcal{Y}}}\left(\pi_{*} \mathcal{O}_{\mathcal{X}}, \mathcal{O}_{\mathcal{Y}}\right)
$$

where $\pi_{*} \mathcal{O}_{\mathcal{X}}$ is placed in degree 0 and where, for local sections $x_{1}, x_{2} \in \pi_{*} \mathcal{O}_{\mathcal{X}}(U)$,

$$
\operatorname{Tr}\left(x_{1}\right)\left(x_{2}\right)=\sum_{g \in G} g\left(x_{1}\right) \cdot g\left(x_{2}\right) .
$$

We then use the inclusion map $\mathcal{O}_{\mathcal{Y}} \hookrightarrow \mathcal{O}_{\mathcal{Y}}\left(\mathcal{Y}_{p}-\mathcal{Y}_{p}^{\text {red }}\right)$ to define a further complex,

$$
E^{\bullet}: \pi_{*} \mathcal{O}_{\mathcal{X}} \stackrel{T r^{\prime}}{\longrightarrow} \operatorname{Hom}_{\mathcal{O}_{\mathcal{Y}}}\left(\pi_{*} \mathcal{O}_{\mathcal{X}}, \mathcal{O}_{\mathcal{Y}}\left(\mathcal{Y}_{p}-\mathcal{Y}_{p}^{\text {red }}\right)\right) \text {. }
$$

The following is a standard result for tame extensions of valuation rings.

Lemma 7.5. Let $N$ be a finite extension of $\mathbb{Q}_{p}$, let $O_{N}$ denote the valuation ring of $N$ and let $\psi$ denote the character of a finitely generated $O_{N}[G]$-module $W$. Let $E_{W}^{\bullet}=\left(W \otimes_{\mathbb{Z}_{p}} E^{\bullet}\right)^{G}$ and $E_{1, W}^{\bullet}=\left(W \otimes_{\mathbb{Z}_{p}} E_{1}^{\bullet}\right)^{G}$ as complexes of $\mathcal{O}_{\mathcal{Y}^{\prime}}$ modules, where $\mathcal{Y}^{\prime}=O_{N} \otimes_{\mathbb{Z}_{p}} \mathcal{Y}$. Then

$$
\operatorname{det}\left(E_{1, W}^{\bullet}\right)=\mathcal{O}_{\mathcal{Y}^{\prime}}\left(-\eta^{-1}\left(T_{1}\right)\right) \quad \text { and } \operatorname{det}\left(E_{W}^{\bullet}\right)=\mathcal{O}_{\mathcal{Y}^{\prime}}\left(-\eta^{-1}(T)\right),
$$

where

$$
T_{1}=\sum_{i}\left(\operatorname{Ind}_{I_{i}}^{G} u_{i}, \psi\right) b_{i} \quad \text { and } \quad T=\psi(1)\left(\mathcal{Y}_{p}^{\mathrm{red}}-\mathcal{Y}_{p}\right)+T_{1}
$$

and $\eta: \mathcal{Y}^{\prime} \rightarrow \mathcal{Y}$ is the projection. 


\section{Pfaffians And Hodge Discriminants}

Proof. First recall that via the trace we can identify $\operatorname{Hom}_{\mathcal{O}_{\mathcal{Y}}}\left(\pi_{*} \mathcal{O}_{\mathcal{X}}, \mathcal{O}_{\mathcal{Y}}\right)$ with the inverse different $\pi_{*} \mathcal{D}_{\mathcal{X} / \mathcal{Y}}^{-1}$. Clearly the result can be proved by consideration of the codimension 1 points of $\mathcal{Y}$. Next let $\eta_{i}$ be the generic point of $b_{i}$ and let $k_{i}$ denote the residue field of $\eta_{i}$. The result therefore follows from the $k_{i}[G]$-isomorphism (see for instance [Tay84, §3.3] for a proof based on an idea due to Chase)

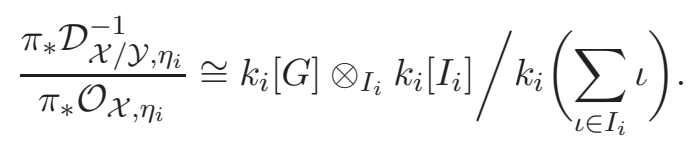

This then establishes the first equality and the second one follows immediately from the first.

Since $\mathcal{X} / \mathcal{Y}$ satisfies (T1) and (T2) we know that, for each $r, 0 \leqslant r \leqslant d$,

$$
\Omega_{\mathcal{X} / \mathbb{Z}_{p}}^{r}\left(\log \mathcal{X}_{p}^{\text {red }} / \log \mathbb{F}_{p}\right)=\pi^{*}\left(\Omega_{\mathcal{Y} / \mathbb{Z}_{p}}^{r}\left(\log \mathcal{Y}_{p}^{\text {red }} / \log \mathbb{F}_{p}\right)\right)
$$

From [CPT02, Lemma 7.10] we recall that, writing $\omega_{\mathcal{Y} / \mathbb{Z}_{p}}$ for the canonical divisor of $\mathcal{Y} / \mathbb{Z}_{p}$, the following lemma holds.

Lemma 7.6. One has

$$
\Omega_{\mathcal{Y} / \mathbb{Z}_{p}}^{d}\left(\log \mathcal{Y}_{p}^{\mathrm{red}} / \log \mathbb{F}_{p}\right)=\omega_{\mathcal{Y} / \mathbb{Z}_{p}}\left(\mathcal{Y}_{p}^{\mathrm{red}}-\mathcal{Y}_{p}\right)
$$

This gives isomorphisms

$$
\Omega_{\mathcal{Y} / \mathbb{Z}_{p}}^{r}\left(\log \mathcal{Y}_{p}^{\text {red }} / \log \mathbb{F}_{p}\right)=\operatorname{Hom}_{\mathcal{O}_{\mathcal{Y}}}\left(\Omega_{\mathcal{Y} / \mathbb{Z}_{p}}^{d-r}\left(\log \mathcal{Y}_{p}^{\text {red }} / \log \mathbb{F}_{p}\right), \omega_{\mathcal{Y}}\left(\mathcal{Y}_{p}^{\text {red }}-\mathcal{Y}_{p}\right)\right)
$$

for $0 \leqslant r \leqslant d$. Tensoring $E^{\bullet}$ with $\Omega_{\mathcal{Y} / \mathbb{Z}_{p}}^{r}\left(\log \mathcal{Y}_{p}^{\text {red }} / \log \mathbb{F}_{p}\right)$ and using the above lemma together with (7.6) and (7.7), we obtain complexes

$$
D_{r}^{\bullet}: \pi_{*} \Omega_{\mathcal{X} / \mathbb{Z}_{p}}^{r}\left(\log \mathcal{X}_{p}^{\text {red }} / \log \mathbb{F}_{p}\right) \stackrel{\delta^{r}}{\longrightarrow} \operatorname{Hom}_{\mathcal{O}_{\mathcal{Y}}}\left(\pi_{*} \Omega_{\mathcal{X} / \mathbb{Z}_{p}}^{d-r}\left(\log \mathcal{X}_{p}^{\text {red }} / \log \mathbb{F}_{p}\right), \omega_{\mathcal{Y} / \mathbb{Z}_{p}}\right) .
$$

In the sequel we shall also be interested in the duality maps on cohomology. To be more precise, we let $X$ denote the generic fiber $\mathcal{X} \times \mathbb{Q}_{p}$ and note that

$$
\Omega_{\mathcal{X} / \mathbb{Z}_{p}}^{r}\left(\log \mathcal{X}_{p}^{\mathrm{red}} / \log \mathbb{F}_{p}\right) \otimes \mathbb{Q}_{p}=\Omega_{X / \mathbb{Q}_{p}}^{r}
$$

Therefore the duality pairing $\delta^{r}$ in (7.8) together with duality on $\mathcal{Y}$ induces a quasi-isomorphism

$$
R \Gamma\left(\delta^{r}\right)_{\mathbb{Q}_{p}}: R \Gamma\left(\pi_{*} \Omega_{X}^{r}\right) \cong \operatorname{Hom}\left(R \Gamma\left(\pi_{*} \Omega_{X}^{d-r}\right), \mathbb{Q}_{p}\right)[-d] .
$$

The induced maps on cohomology coincide with $\# G$ times the Serre duality maps $\sigma_{i, j}$ defined in (3.5) of $\S 3.1$ when $\mathbb{Q}$ is replaced by $\mathbb{Q}_{p}$. (The $\# G$ factor arises from the fact that in (3.5) we multiplied the usual trace map by $1 / \# G$.) Define $Y=X / G=\mathcal{Y} \times \mathbb{Q}_{p}$ and

$$
e(Y)=\sum_{i, j}(-1)^{i+j} \operatorname{dim}_{\mathbb{Q}_{p}} H^{j}\left(Y, \Omega_{Y / \mathbb{Q}_{p}}^{i}\right) .
$$

7.2.2 Non-archimedean Pfaffians. In this section we introduce the non-archimedean counterpart to the invariant sgn.pf of $\S 2.4$. Again let $N$ be a finite extension of $\mathbb{Q}_{p}$ and let $W$ be a symplectic representation of $G$ defined over $N$, with $G$-invariant alternating form $\kappa$ and with character $\psi$. Suppose that $\mathcal{F}$ is a coherent $G$-sheaf on $\mathcal{X}$. Since the action of $G$ on $\mathcal{X}$ is tame, $R \Gamma(\mathcal{F})$ is quasi-isomorphic to a bounded complex $Q^{\bullet}$ of finitely generated projective $\mathbb{Z}_{p}[G]$-modules. We can furthermore assume that all but one of the terms of $Q^{\bullet}$ are free $\mathbb{Z}_{p}[G]$-modules. The action of $G$ on the general fiber $X$ of $\mathcal{X}$ is free. Hence by the Lefschetz-Riemann-Roch theorem, the character of the virtual $\mathbb{Q}_{p}[G]$-module $\sum_{i}(-1)^{i}\left[\mathbb{Q}_{p} \otimes_{\mathbb{Z}_{p}} Q^{i}\right]$ is the character of a free module. This forces all the $Q^{i}$ to be free $\mathbb{Z}_{p}[G]$-modules, since projective $\mathbb{Z}_{p}[G]$-modules are determined by their characters. 


\section{T. Chinburg, G. Pappas and M. Taylor}

We can thus find a bounded complex $P^{\bullet}$ of finitely generated free $\mathbb{Z}_{p}[G]$-modules which is quasiisomorphic to $R \Gamma\left(\bigoplus_{i} \Omega_{\mathcal{X} / \mathbb{Z}_{p}}^{i}\left(\log \mathcal{X}_{p}^{\text {red }} / \log \mathbb{F}_{p}\right)[d-i]\right)$. The modules $P^{\text {ev }}$ and $P^{\text {odd }}$ are then defined as in $\S 2.3$. As in $\S 2.7$ we fix free $\mathbb{Z}_{p}$-modules $F^{\mathrm{ev}}$ and $F^{\text {odd }}$ which support $\mathbb{Z}_{p}[G]$-isomorphisms

$$
\mathbb{Z}_{p}[G] \otimes F^{\mathrm{ev}} \cong P^{\mathrm{ev}}, \quad \mathbb{Z}_{p}[G] \otimes F^{\mathrm{odd}} \cong P^{\text {odd }},
$$

so that we have isomorphisms

$$
W \otimes F^{\mathrm{ev}} \cong\left(W \otimes P^{\mathrm{ev}}\right)^{G}=P_{W}^{\mathrm{ev}}, \quad W \otimes F^{\mathrm{odd}} \cong\left(W \otimes P^{\mathrm{odd}}\right)^{G}=P_{W}^{\mathrm{odd}} .
$$

We have

$$
\operatorname{rank}_{\mathbb{Z}_{p}}\left(F^{\mathrm{ev}}\right)-\operatorname{rank}_{\mathbb{Z}_{p}}\left(F^{\text {odd }}\right)=\operatorname{rank}_{\mathbb{Z}_{p}[G]}\left(P^{\mathrm{ev}}\right)-\operatorname{rank}_{\mathbb{Z}_{p}[G]}\left(P^{\text {odd }}\right)=(-1)^{d} e(Y) .
$$

We set $\operatorname{det} F^{\bullet}=\operatorname{det} F^{\mathrm{ev}} \otimes \operatorname{det} F^{\text {odd }}{ }^{-1}$. Let $\sigma^{\mathrm{ev}}, \sigma^{\text {odd }}$ be the duality pairings of $\S 3.1$, and let $\operatorname{Pf}_{W}$ denote the composite isomorphism:

$$
\begin{aligned}
\operatorname{det}\left(F^{\bullet}\right)^{\operatorname{dim} W} \otimes N & \cong \operatorname{det}\left(F^{\bullet}\right)^{\operatorname{dim} W} \otimes \operatorname{det} W^{\operatorname{dim} F^{\bullet}} \\
& \cong \operatorname{det}\left(W \otimes F^{\mathrm{ev}}\right) \otimes \operatorname{det}\left(W \otimes F^{\mathrm{odd}}\right)^{-1} \\
& \cong \operatorname{det} P_{W}^{\mathrm{ev}} \otimes \operatorname{det} P_{W}^{\mathrm{odd}}-1 \\
& \cong \operatorname{det} P_{W}^{\bullet} \\
& \cong N
\end{aligned}
$$

where the first isomorphism is induced by the tensor power of the inverse of the Pfaffian isomorphism

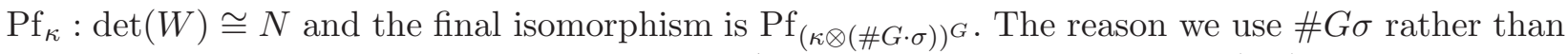
$\sigma$ in the final automorphism is to remove the $1 / \# G$ factor on the far right in (3.5) of $\S 3.1$, so as to use the standard trace map coming from duality on the general fiber of $\mathcal{X}$.

The following result is proved in the same way as Proposition 2.6.

Proposition 7.7. Let $\psi$ be the character of $W$. Let $|\operatorname{Pf}(\mathcal{X}, \psi)|_{p} \in \mathbb{R}\left(\right.$ respectively $\left.\|\operatorname{Pf}(\mathcal{X}, \psi)\|_{p} \in \lambda^{\mathbb{Q}}\right)$ be the result of evaluating the above map $\operatorname{Pf}_{W}$ on the $\operatorname{dim}(W)$ th power of the determinant of any choice of $\mathbb{Z}_{p}$-bases of $F^{\mathrm{ev}}$ and $F^{\text {odd }}$ and by then apply the $p$-adic absolute value function $|\cdot|_{p}: N^{*} \rightarrow \mathbb{R}$ (respectively the function \|\|$_{p}: N^{*} \rightarrow \lambda^{\mathbb{Q}}$ ). Then $|\operatorname{Pf}(\mathcal{X}, \psi)|_{p}$ and $\|\operatorname{Pf}(\mathcal{X}, \psi)\|_{p}$ are independent of the choice of basis, the choice of isomorphisms (7.11) and the choice of a symplectic representation $W$ with character $\psi$.

To compute Pfaffians using discriminants coming from duality pairings, we need the following linear algebra result. Let $O_{N}$ be the valuation ring of $N$. Suppose $\mathcal{W}$ is a finitely generated $O_{N^{-}}$ submodule of $W$ such that the restriction of $\kappa$ to $\mathcal{W}$ is perfect, in the sense that it induces an isomorphism from $\mathcal{W}$ to $\mathcal{W}^{D}=\operatorname{Hom}_{O_{N}}\left(\mathcal{W}, O_{N}\right)$. Suppose that $P$ is a finitely generated free $\mathbb{Z}_{p}[G]$ module and that $\tau: P \rightarrow P^{D}=\operatorname{Hom}_{\mathbb{Z}_{p}}\left(P, \mathbb{Z}_{p}\right)$ is a homomorphism which is an isomorphism on tensoring with $\mathbb{Q}_{p}$. Then $\tau$ induces an $O_{N}$-module homomorphism

$$
\tau_{\mathcal{W}}: P_{\mathcal{W}}=\left(\mathcal{W} \otimes_{\mathbb{Z}_{p}} P\right)^{G} \rightarrow\left(\mathcal{W} \otimes \operatorname{Hom}_{\mathbb{Z}_{p}}\left(P, \mathbb{Z}_{p}\right)\right)^{G}=\left(P^{D}\right)_{\mathcal{W}}
$$

which is injective with cokernel a finite $O_{N}$-module with some non-zero order ideal $I \subset O_{N}$. Let

$$
s_{\mathcal{W}}:\left(\mathcal{W} \otimes \operatorname{Hom}_{\mathbb{Z}_{p}}\left(P, \mathbb{Z}_{p}\right)\right)^{G}=\left(P^{D}\right)_{\mathcal{W}} \rightarrow\left(P_{\mathcal{W}}\right)^{D}=\operatorname{Hom}_{O_{N}}\left(P_{\mathcal{W}}, O_{N}\right)
$$

be the homomorphism which is the composition of the isomorphism

$$
\left(\mathcal{W} \otimes \operatorname{Hom}_{\mathbb{Z}_{p}}\left(P, \mathbb{Z}_{p}\right)\right)^{G}=\left(\operatorname{Hom}_{O_{N}}\left(\mathcal{W}, O_{N}\right) \otimes \operatorname{Hom}_{\mathbb{Z}_{p}}\left(P, \mathbb{Z}_{p}\right)\right)^{G} \rightarrow \operatorname{Hom}_{O_{N}}\left(\mathcal{W} \otimes P, O_{N}\right)^{G}
$$

resulting from the $G$-isomorphism $\kappa: \mathcal{W} \rightarrow \mathcal{W}^{D}=\operatorname{Hom}\left(\mathcal{W}, O_{N}\right)$ with the map resulting from restricting homomorphisms from $\mathcal{W} \otimes P$ to $P_{W}=(\mathcal{W} \otimes P)^{G}$. Let $h: P_{\mathcal{W}} \times P_{\mathcal{W}} \rightarrow O_{N}$ be the bilinear form associated to the map $s_{\mathcal{W}} \circ \tau_{\mathcal{W}}: P_{\mathcal{W}} \rightarrow\left(P_{\mathcal{W}}\right)^{D}$. 


\section{Pfaffians AND Hodge DiscRiminants}

Lemma 7.8. Let $r$ be the rank of $P$ as a $\mathbb{Z}_{p}[G]$-module and let $z$ be the rank of $\mathcal{W}$ as an $O_{N}$-module.

(a) The map $s_{\mathcal{W}}$ induces an isomorphism from $\left(P^{D}\right)_{\mathcal{W}}$ to $\# G \cdot\left(P_{\mathcal{W}}\right)^{D}$.

(b) Let $\beta$ be an $O_{N}$-module generator for $\operatorname{det}_{O_{N}}\left(P_{\mathcal{W}}\right)$. Then $\lambda=\operatorname{det}\left(\tau_{W}\right)(\beta) \otimes \beta^{-1}$ is a non-zero element of

$$
\operatorname{det}\left(\operatorname{Cone}\left(\tau_{\mathcal{W}}\right)\right)=\operatorname{det}_{O_{N}}\left(\left(P^{D}\right) \mathcal{W}\right) \otimes\left(\operatorname{det}_{O_{N}}\left(P_{\mathcal{W}}\right)\right)^{\otimes(-1)}
$$

(c) The cokernel of $s_{\mathcal{W}} \circ \tau_{\mathcal{W}}$ has $O_{N}$-order ideal

$$
(\# G)^{r z} \cdot I=d_{h}\left(\beta^{\otimes 2}\right) \cdot O_{N},
$$

where $d_{h}: \operatorname{det}\left(P_{\mathcal{W}}\right)^{\otimes 2} \rightarrow N$ is the discriminant associated to the bilinear form $h$.

(d) One has

$$
\operatorname{det}\left(\operatorname{Cone}\left(\tau_{\mathcal{W}}\right)\right)=I^{-1} \cdot \lambda=(\# G)^{r z} \cdot d_{h}\left(\beta^{\otimes 2}\right)^{-1} \cdot \lambda
$$

Thus if ||$_{p}$ is the $p$-adic absolute value on $\operatorname{det}\left(\operatorname{Cone}\left(\tau_{\mathcal{W}}\right)\right)$ for which $|\lambda|_{p}=1$, and $\alpha$ is a generator for $\operatorname{det}\left(\operatorname{Cone}\left(\tau_{\mathcal{W}}\right)\right)$ as an $O_{N}$-module, then

$$
\left|(\# G)^{r z}\right|_{p} \cdot|\alpha|_{p}^{-1}=\left|d_{h}\left(\beta^{\otimes 2}\right)\right|_{p} .
$$

Proof. To show part (a), note that $\operatorname{Hom}_{O_{N}}\left(\mathcal{W} \otimes P, O_{N}\right)^{G}=\operatorname{Hom}_{O_{N}}\left((\mathcal{W} \otimes P)_{G}, O_{N}\right)$, where $(\mathcal{W} \otimes P)_{G}$ is the $O_{N}$-module of $G$-covariants of $\mathcal{W} \otimes P$. Since $P$ is finitely generated and free, the map induced by the inclusion of $(\mathcal{W} \otimes P)^{G}$ into $\mathcal{W} \otimes P$ gives an isomorphism $P_{\mathcal{W}} \rightarrow \# G \cdot(\mathcal{W} \otimes P)_{G}$, which leads to part (a). Since $P$ is a free $\mathbb{Z}_{p}[G]$-module, the rank of $P_{\mathcal{W}}$ over $O_{N}$ is $r z$. This implies parts (b) and (c). Part (d) now follows from parts (b) and (c) together with the fact that $\operatorname{det}_{O_{N}}\left(\left(P^{D}\right)_{\mathcal{W}}\right)=$ $I^{-1} \cdot \operatorname{det}\left(\tau_{\mathcal{W}}\right)(\beta)$ by the definition of $I$.

Proposition 7.9. Let $\mathcal{W}$ be as in Lemma 7.8 and let $\psi$ be the (symplectic) character of $W=$ $N \otimes_{O_{N}} \mathcal{W}$. Let $\delta^{\bullet}$ denote the shifted direct sum $\delta^{\bullet}=\bigoplus_{r} \delta^{r}[d-r]$ of the morphisms appearing in (7.8). The complex $R \Gamma\left(\text { Cone }\left(\delta^{\bullet}\right)\right)_{\mathcal{W}}$ is a perfect complex of $O_{N}$-modules, with finite cohomology groups, and there is a canonical trivialization $N \otimes_{O_{N}} \operatorname{det}\left(R \Gamma\left(\operatorname{Cone}\left(\delta^{\bullet}\right)\right)_{\mathcal{W}}\right)=N$ arising from the duality theorem on the general fiber $X$ of $\mathcal{X}$. This trivialization and the $p$-adic absolute value ||$_{p}$ on $N$ give an absolute value on $\operatorname{det}\left(R \Gamma\left(\operatorname{Cone}\left(\delta^{\bullet}\right)\right)_{\mathcal{W}}\right)$, which we also denote by $\left.\right|_{p}$. We have

$$
\left|(\# G)^{(-1)^{d} e(Y) \operatorname{dim}(W)}\right|_{p} \cdot|\operatorname{Pf}(\mathcal{X}, \psi)|_{p}^{-2}=\left|\operatorname{det}\left(R \Gamma\left(\operatorname{Cone}\left(\delta^{\bullet}\right)\right)_{\mathcal{W}}\right)\right|_{p}
$$

where the right-hand side is defined to be $|\alpha|_{p}$ for any $O_{N}$ generator $\alpha$ of $\operatorname{det}\left(R \Gamma\left(\operatorname{Cone}\left(\delta^{\bullet}\right)\right)_{\mathcal{W}}\right)$.

Proof. Recall from $\S 2.2$ that for a finite-dimensional $K$-vector space $V$ which supports an alternating form $h$, then $d_{h}= \pm \mathrm{Pf}_{h}^{\otimes 2}$ on $\operatorname{det} V^{\otimes 2}$, where $d_{h}$ is the discriminant functional. We apply this observation to the following situation. Suppose that $P^{\bullet}$ denotes a bounded complex of free $\mathbb{Z}_{p}[G]$-modules with $G$-invariant symmetric forms $\sigma^{\text {ev }}$ (respectively $\sigma^{\text {odd }}$ ) on $\mathrm{H}^{\mathrm{ev}}\left(P^{\bullet}\right)$ (respectively $\left.\mathrm{H}^{\text {odd }}\left(P^{\bullet}\right)\right)$. Then $d_{\left(\kappa \otimes \sigma^{\mathrm{ev}}\right)^{G}}= \pm \operatorname{Pf}_{\left(\kappa \otimes \sigma^{\text {ev }}\right)^{G}}^{\otimes 2}$ on $\operatorname{det} \mathrm{H}^{\mathrm{ev}}\left(P_{W}^{\bullet}\right)^{\otimes 2}$ and $d_{\left(\kappa \otimes \sigma^{\text {odd }}\right)^{G}}= \pm \operatorname{Pf}_{\left(\kappa \otimes \sigma^{\text {odd }}\right)^{G}}^{\otimes 2}$ on $\operatorname{det} \mathrm{H}^{\text {odd }}\left(P_{W}^{\bullet}\right)^{\otimes 2}$.

We now specialize to the case in which $P^{\bullet}$ is a complex of free $\mathbb{Z}_{p}[G]$-modules which is quasiisomorphic to

$$
R \Gamma\left(\bigoplus_{i} \Omega_{\mathcal{X} / \mathbb{Z}_{p}}^{i}\left(\log \mathcal{X}_{p}^{\mathrm{red}} / \log \mathbb{F}_{p}\right)[d-i]\right)
$$

and $\sigma^{\text {ev }}$ (respectively $\sigma^{\text {odd }}$ ) are the duality pairings of $\S 3.1$. Then the duality map $\delta^{\bullet}$ gives a map

$$
R \Gamma\left(\delta^{\bullet}\right): P^{\bullet} \rightarrow \operatorname{Hom}\left(P^{\bullet}, \mathbb{Z}_{p}\right) .
$$

As noted after (7.9), this map tensored with $\mathbb{Q}_{p}$ over $\mathbb{Z}_{p}$ induces the Serre duality pairings on cohomology which determine $\# G \sigma^{\mathrm{ev}}$ and $\# G \sigma^{\text {odd }}$. From $(7.15)$ we get a map

$$
R \Gamma\left(\delta^{\bullet}\right)_{\mathcal{W}}: P_{\mathcal{W}}^{\bullet} \rightarrow \operatorname{Hom}\left(P^{\bullet}, O_{N}\right)_{\mathcal{W}}
$$




\section{T. Chinburg, G. Pappas and M. Taylor}

such that

$$
R \Gamma\left(\operatorname{Cone}\left(\delta^{\bullet}\right)\right)_{\mathcal{W}}=\operatorname{Cone}\left(R \Gamma\left(\delta^{\bullet}\right) \mathcal{W}\right) .
$$

To complete the proof we now choose $\mathbb{Z}_{p}[G]$-bases $\left\{a_{j}^{i}\right\}$ of each term $P^{i}$ of $P^{\bullet}$ and a hyperbolic basis $\left\{w_{k}\right\}$ of $W$; we then let $b=\bigotimes_{i}\left(\bigwedge_{j, k} a_{i}^{j} \otimes w_{k}\right)^{(-1)^{i}}$. Then by definition

$$
\begin{aligned}
|\operatorname{Pf}(\mathcal{X}, \psi)|_{p}^{2} & =\left|\operatorname{Pf}_{(\kappa \otimes(\# G \cdot \sigma))^{G}}\left(\xi_{W}(b)\right)\right|_{p}^{2} \\
& =\left| \pm d_{(\kappa \otimes(\# G \cdot \sigma))^{G}}\left(\xi_{W}(b)^{\otimes^{2}}\right)\right|_{p} \\
& =\left|(\# G)^{(-1)^{d} e(Y) \operatorname{dim}(W)}\right|_{p} \cdot\left|\operatorname{det}\left(R \Gamma\left(\operatorname{Cone}\left(\delta^{\bullet}\right)\right)_{\mathcal{W}}\right)\right|_{p}^{-1},
\end{aligned}
$$

where the last equality follows from part (d) of Lemma 7.8 together with (7.12).

\subsection{Proof of Theorem 1.2}

In this section we shall use the Riemann-Roch theorem for localized Chern characters [Ful98] to show the following result.

Proposition 7.10. Suppose $p$ does not divide $\# G$. Let $\|\operatorname{Pf}(\mathcal{X})\|_{p} \in \operatorname{Hom}\left(R_{G}^{\mathrm{s}}, J_{f}(\bar{L})\right)$ be the character function which sends each $\psi \in R_{G}^{\mathrm{s}}$ to the idele having semi-local component in $L_{l}$ equal to 1 if $l \neq p$ and equal to $\|\operatorname{Pf}(\mathcal{X}, \psi-\operatorname{dim}(\psi) \cdot 1)\|_{p}$ if $l=p$. Then for each $\psi \in R_{G}^{\mathrm{s}}$ one has

$$
\left(\|\operatorname{Pf}(\mathcal{X})\|_{p}(\psi)\right)_{p}^{(-1)^{d}}=k(\psi)_{p}^{-1}=\left\|\widetilde{\varepsilon}_{0}^{\mathrm{s}}(\mathcal{X})\right\|_{p}(\psi) .
$$

In consequence,

$$
\|\operatorname{Pf}(\mathcal{X}, \psi-\psi(1) \cdot 1)\|_{p}=(-1)^{a_{p}(\psi)}|\operatorname{Pf}(\mathcal{X}, \psi-\psi(1) \cdot 1)|_{p} \in(-p)^{\mathbb{Z}},
$$

where $a_{p}(\psi)=v_{p}\left(|\operatorname{Pf}(\mathcal{X}, \psi-\psi(1) \cdot 1)|_{p}\right)$. Hence $\|\operatorname{Pf}(\mathcal{X})\|_{p} \in \operatorname{Hom}_{\Omega_{L}}\left(R_{G}^{\mathrm{s}}, J_{f}(\bar{L})\right)$.

Remark. The hypothesis that $p \nmid \# G$ is not essential to the method, e.g. it was not used in Proposition 7.9. We assume that $p \nmid \# G$ in order to apply the calculations in [CPT07, Theorem 3.10(e)]. The second equality in (7.16) was shown in Proposition 7.3(c); we include it here for the sake of completeness. Note that by Proposition 7.2 , the information contained in $\left\|\tilde{\varepsilon}_{0}^{\mathrm{s}}(\mathcal{X})\right\|_{p}(\psi)$ is the $p$-adic absolute value of $\tilde{\varepsilon}_{0}^{\mathrm{s}}(\mathcal{X})(\psi)$. Theorem 7.11 (below) presents a sharper result because it also captures the sign of $\tilde{\varepsilon}_{0}^{\mathrm{s}}(\mathcal{X})(\psi)$.

Before proving this proposition, let us first use it to establish the following result, which proves Theorem 1.2 of the Introduction.

Theorem 7.11. The class in $\operatorname{AdHCL}_{f}\left(O_{L}[G]\right)$ represented by $\|\operatorname{Pf}(\mathcal{X})\|_{p}^{-1}$ equals $c^{(-1)^{d}}$ when $c$ is the rational class in $\operatorname{AdHCL}_{f}\left(O_{L}[G]\right)$ defined in Definition 7.1. Hence by (7.4) of Theorem 7.4, $\|\operatorname{Pf}(\mathcal{X})\|_{p}$ and $d=\operatorname{dim}(\mathcal{Y})-1$ determine the constants $\varepsilon_{0}(\mathcal{Y}, \psi) \in \mathbb{Q}^{*}$ for all virtual symplectic characters $\psi$ of degree 0 .

Proof. This follows from Proposition 7.10 together with the definition of the class $c$ in terms of the function $\psi \rightarrow k(\psi)$ which was given in Definition 7.1.

We now return to the proof of Proposition 7.10.

Proof of Proposition 7.10. As previously, let $N$ be a finite extension of $\mathbb{Q}_{p}$, and let $O_{N}$ denote the valuation ring of $N$. Let $\mathcal{V}$ denote an $O_{N}[G]$-module with character $\psi$; set $\mathcal{W}=\mathcal{V} \oplus \mathcal{V}^{D}$ and we endow $\mathcal{W}$ with the $G$-invariant alternating form $\kappa$ given by the rule

$$
\kappa\left((v, f),\left(v^{\prime}, f^{\prime}\right)\right)=f^{\prime}(v)-f\left(v^{\prime}\right) .
$$

Since $\mathcal{W}$ has character $2 \psi$, by additivity and by Proposition 7.9 it will suffice to show that

$$
\left|\operatorname{det}\left(R \Gamma\left(\operatorname{Cone}\left(\delta^{\bullet}\right)\right)_{\mathcal{W}}\right)\right|_{p}^{(-1)^{d}}=k(2 \psi)_{p}^{2} .
$$




\section{Pfaffians AND Hodge DiscRiminants}

To show this equality we let $f: \mathcal{Y} \rightarrow \operatorname{Spec}\left(\mathbb{Z}_{p}\right)$ denote the structure map of $\mathcal{Y}$. With the notation of (7.8) we have

$$
D_{r, \mathcal{W}}^{\bullet}=E_{\mathcal{W}}^{\bullet} \otimes \Omega_{\mathcal{Y} / \mathbb{Z}_{p}}^{r}\left(\log \mathcal{Y}_{p}^{\text {red }} / \log \mathbb{F}_{p}\right)
$$

This is a complex of $O_{N} \otimes_{\mathbb{Z}_{p}} O_{\mathcal{Y}}$-modules on $\mathcal{Y}$; we will treat it simply as a complex of $O_{\mathcal{Y}^{-}}$ modules in the following Riemann-Roch arguments. We must recall some notation from [Ful98]. Let $A^{*}\left(\mathcal{Y}_{p} \rightarrow \mathcal{Y}\right)_{\mathbb{Q}_{p}}$ denote the group of bivariant classes as defined in [Ful98, § 17.1]. The complex $D_{r, \mathcal{W}}^{\bullet}$ is exact off $\mathcal{Y}_{p}$ so it has a localized Chern character $\operatorname{ch}_{\mathcal{Y}_{p}}^{\mathcal{Y}}\left(D_{r, \mathcal{W}}^{\bullet}\right)$ (see [Ful98, §18.1]). Let $\operatorname{Td}(f)=\operatorname{td}(f)[Y]$ denote the Todd class associated to the (virtual) tangent bundle of $\mathcal{Y}$ (see [Ful98, $\S 18.2]$ ). Write $D^{\bullet}$ for the shifted direct sum $\bigoplus \delta^{r}[d-r]$. The following 'localized' Riemann-Roch theorem follows from [Rob98, Theorems 12.5.1 and 12.6.1]:

$$
v_{p, N}\left(\operatorname{det}\left(R \Gamma\left(\operatorname{Cone}\left(\delta^{\bullet}\right)\right)_{\mathcal{W}}\right)\right)=f_{p, *}\left(\left(\left(\operatorname{ch}_{\mathcal{Y}_{p}}^{\mathcal{Y}}\left(D_{\bullet}^{\bullet}, \mathcal{W}\right)\right) \cap \operatorname{Td}(f)\right)_{0}\right) .
$$

In this equality, the map $f_{p, *}: \mathbb{Q} \otimes_{\mathbb{Z}} A_{0}\left(\mathcal{Y}_{p}\right) \rightarrow \mathbb{Q} \otimes_{\mathbb{Z}} A_{0}\left(\operatorname{Spec}\left(\mathbb{F}_{p}\right)\right)=\mathbb{Q}$ on the right-hand side is the push forward of 0-cycles, so that it is given by the degree of 0-cycles over $\mathbb{F}_{p}$. Since we are working over $\mathbb{Z}_{p}$ rather than over $O_{N}$, the left-hand side of (7.20) is defined to be $v_{p, N}(\alpha)$ for any $O_{N}$ generator $\alpha$ of $\operatorname{det}\left(R \Gamma\left(\operatorname{Cone}\left(\delta_{\mathcal{W}}^{\bullet}\right)\right)\right)$, where $v_{p, N}: N^{*} \rightarrow \mathbb{Z}$ is the valuation normalized by $v_{p, N}(p)=\left[N: \mathbb{Q}_{p}\right]$. Equality (7.20) can also be derived following the proof of [Ful98, Theorem 18.2(1)] by considering the morphism $f_{p}: \mathcal{Y}_{p} \rightarrow \operatorname{Spec}\left(\mathbb{F}_{p}\right)$ as a morphism of schemes over $S=\operatorname{Spec}\left(\mathbb{Z}_{p}\right)$.

On the one hand by (7.19) above and [CPT07, Theorem 3.10(e)] we know that

$$
\begin{aligned}
\operatorname{ch}_{\mathcal{Y}_{p}}^{\mathcal{Y}}\left(D_{\bullet, \mathcal{W}}^{\bullet}\right) & =\operatorname{ch}_{\mathcal{Y}_{p}}^{\mathcal{Y}}\left(E_{\mathcal{W}}^{\bullet}\right) \cdot \operatorname{ch}\left(\sum_{r=0}^{d}(-1)^{d-r} \Omega_{\mathcal{Y} / \mathbb{Z}_{p}}^{r}\left(\log \mathcal{Y}_{p}^{\text {red }} / \log \mathbb{F}_{p}\right)\right) \\
& =(-1)^{d} \operatorname{ch}_{\mathcal{Y}_{p}}^{\mathcal{Y}}\left(E_{\mathcal{W}}^{\bullet}\right) \cdot \operatorname{ch}\left(\lambda_{-1}\left(\Omega_{\mathcal{Y} / \mathbb{Z}_{p}}^{1}\left(\log \mathcal{Y}_{p}^{\text {red }} / \log \mathbb{F}_{p}\right)\right)\right) .
\end{aligned}
$$

On the other hand from Lemma 7.5 and [CPT07, Theorem 3.10] we deduce that for an $O_{N}[G]$ module $\mathcal{W}$ with symplectic character $2 \psi$ one has

$$
\operatorname{ch}_{\mathcal{Y}_{p}}^{\mathcal{Y}}\left(E_{\mathcal{W}}^{\bullet}\right) \equiv-\left[N: \mathbb{Q}_{p}\right] \sum_{i}\left(\operatorname{Ind}_{I_{i}}^{G} u_{i}, 2 \psi\right) b_{i} \quad \bmod A^{>1}\left(\mathcal{Y}_{p} \rightarrow \mathcal{Y}\right)_{\mathbb{Q}_{p}}
$$

Here the $\left[N: \mathbb{Q}_{p}\right]$ factor on the right comes from the fact that (7.5) in Lemma 7.5 refers to $\mathcal{Y}^{\prime}=O_{N} \otimes \mathcal{Y}$ rather than $\mathcal{Y}$. Since $\lambda_{-1}\left(\Omega_{\mathcal{Y} / \mathbb{Z}_{p}}^{1}\left(\log \mathcal{Y}_{p}^{\text {red }} / \log \mathbb{F}_{p}\right)\right)$ lies in the $d$ th level of the $\gamma$-filtration of $\mathrm{K}_{0}(\mathcal{Y})$, we know that

$$
\operatorname{ch}\left(\lambda_{-1}\left(\Omega_{\mathcal{Y} / \mathbb{Z}_{p}}^{1}\left(\log \mathcal{Y}_{p}^{\mathrm{red}} / \log \mathbb{F}_{p}\right)\right)\right)=\lambda_{-1}\left(\Omega_{\mathcal{Y} / \mathbb{Z}_{p}}^{1}\left(\log \mathcal{Y}_{p}^{\mathrm{red}} / \log \mathbb{F}_{p}\right)\right)
$$

and we also note that trivially

$$
\operatorname{td}(f) \equiv 1 \quad \bmod A^{>0}\left(\mathcal{Y}_{p} \rightarrow \mathcal{Y}\right)_{\mathbb{Q}_{p}}
$$

We can therefore piece the above together to deduce that

$$
\begin{aligned}
& v_{p, N}\left(\operatorname{det}\left(R \Gamma\left(\operatorname{Cone}\left(\delta_{\mathcal{W}}^{\bullet}\right)\right)\right)\right. \\
& \quad=\left[N: \mathbb{Q}_{p}\right](-1)^{d+1} \operatorname{deg}_{\mathbb{F}_{p}}\left(\lambda_{-1}\left(\Omega_{\mathcal{Y} / \mathbb{Z}_{p}}^{1}\left(\log \mathcal{Y}_{p}^{\mathrm{red}} / \log \mathbb{F}_{p}\right)\right) \cdot\left(\sum_{i}\left(\operatorname{Ind}_{I_{i}}^{G} u_{i}, 2 \psi\right) b_{i}\right)\right) .
\end{aligned}
$$

The desired equality (7.18) now follows from (7.21) together with the definitions of $k(\psi)$ in $(7.2)$ and of $k^{\prime}(\psi)$ in $(7.1)$ and the normalization of $v_{p, N}$. 


\section{T. Chinburg, G. Pappas and M. Taylor}

\subsection{An example}

Suppose $L$ is a tamely ramified quadratic extension of $\mathbb{Q}_{p}$. Let $G=\operatorname{Gal}\left(L / \mathbb{Q}_{p}\right)$ act on $\mathcal{X}=\operatorname{Spec}\left(O_{L}\right)$, so that $\mathcal{Y}=\mathcal{X} / G=\operatorname{Spec}\left(\mathbb{Z}_{p}\right)$. Let $N=\mathbb{Q}_{p}$ and let $\mathcal{W}=\mathbb{Z}_{p} v_{1} \oplus \mathbb{Z}_{p} v_{2}$ have alternating form $\kappa: \mathcal{W} \times \mathcal{W} \rightarrow \mathbb{Z}_{p}=O_{N}$ determined by $\kappa\left(v_{1}, v_{2}\right)=1$. Fix an action of $G$ on $\mathcal{W}$ by letting the non-trivial element $g \in G$ act by multiplication by -1 . The character $\psi$ of $W=\mathbb{Q}_{p} \otimes_{\mathbb{Z}_{p}} \mathcal{W}$ is $2 \cdot \chi$ when $\chi$ is the one-dimensional non-trivial character of $G$.

Let $e\left(L / \mathbb{Q}_{p}\right) \in\{1,2\}$ be the ramification degree of $L / \mathbb{Q}_{p}$, where $e\left(L / \mathbb{Q}_{p}\right)=1$ if $p=2$. The Pfaffian divisor $\operatorname{Pf}(\mathcal{Y}, \psi)$ is $\left(e\left(L / \mathbb{Q}_{p}\right)-1\right) b$ when $b$ is the closed point of $\mathcal{Y}$. The constant

$$
\tilde{\varepsilon}_{0}\left(\mathcal{X} \otimes_{G} W\right)=\varepsilon_{0}\left(\mathcal{X} \otimes_{G}(W-2 \cdot 1)\right)
$$

is 1 if $e\left(L / \mathbb{Q}_{p}\right)=1$ and otherwise is $\left(\begin{array}{c}-1 \\ p\end{array}\right) p$. Since $k^{\prime}(\psi)=(-p)^{e\left(L / \mathbb{Q}_{p}\right)-1}$, this is consistent with Proposition 7.3.

The complexes $E^{\bullet}$ and $E_{1}^{\bullet}$ of $\S 7.2 .1$ correspond to the complex $O_{L} \rightarrow \operatorname{Hom}_{\mathbb{Z}_{p}}\left(O_{L}, \mathbb{Z}_{p}\right)$ induced by the trace map $\operatorname{Tr}_{L / \mathbb{Q}_{p}}$. One has $O_{L}=\mathbb{Z}_{p}[G] \cdot w$ for $w=(1+\sqrt{d}) / 2$ and some non-square $d \in \mathbb{Z}_{p}^{*}$; if $p=2$ then $d \equiv 5 \bmod 8$. In $\S 7.2 .2$ we can take $P^{\bullet}$ (respectively $F^{\bullet}$ ) to be the complex having $P=O_{L}$ (respectively $F=\mathbb{Z}_{p} w$ ) in degree 0 and all other terms equal to 0 . The module $P_{\mathcal{W}}=(\mathcal{W} \otimes P)^{G}$ is then identified with $\mathbb{Z}_{p}\left(v_{1} \otimes \sqrt{d}\right) \oplus \mathbb{Z}_{p}\left(v_{2} \otimes \sqrt{d}\right)$, and the pairing $(\kappa \otimes \# G \sigma)^{G}$ is the unique alternating pairing sending $\left(v_{1} \otimes \sqrt{d}, v_{2} \otimes \sqrt{d}\right)$ to $2 d$.

Using the basis $w^{\otimes 2}$ for $F^{\otimes 2}$ in (7.13) leads to $\operatorname{Pf}(\mathcal{X}, \psi)=2 d$. Note that if $L / \mathbb{Q}_{p}$ is unramified, then $E^{\bullet}$ and $E_{1}^{\bullet}$ are acyclic, $R \Gamma\left(\operatorname{Cone}\left(\delta^{\bullet}\right)\right)_{\mathcal{W}}$ is acyclic and $\left|\operatorname{det}\left(R \Gamma\left(\operatorname{Cone}\left(\delta^{\bullet}\right)\right)_{\mathcal{W}}\right)\right|_{p}=1$. In particular, if $p=2$ then $|\operatorname{Pf}(\mathcal{X}, \psi)|_{p}=|2 d|_{p}=|2|_{p}$ in accordance with (7.14).

We now drop the assumption that $L / \mathbb{Q}_{p}$ is unramified, but assume that $p \nmid \# G$. Since $d=0$, and $\| \pm p\|_{p}=(-p)^{-1}$, the above calculations check (7.16) and (7.17) in this case. It follows that the classes $\|\operatorname{Pf}(\mathcal{X})\|_{p}^{-1}$ and $c^{(-1)^{d}}$ in $\operatorname{AdHCL}_{f}\left(O_{L}[G]\right)$ which appear in Theorem 7.11 are both represented by the character function which sends each $\lambda \in R_{G}^{\mathrm{s}}$ to $k(\lambda)$. As noted in Theorem 7.11, these classes determine $\varepsilon_{0}\left(\mathcal{X} \otimes_{G}(W-2 \cdot 1)\right)=\varepsilon(\mathcal{Y}, \psi-2 \cdot 1)$ via Theorem 7.4. Note that if $L / \mathbb{Q}_{p}$ is ramified, then $p \neq 2$, and the above calculations show that $\left\|\tilde{\varepsilon}_{0}(\mathcal{X})\right\|_{p} \cdot \tilde{\varepsilon}_{0}(\mathcal{X})$ in part (a) of Proposition 7.3 takes the value 1 (respectively -1$)$ on $\psi$ if $p \equiv 3 \bmod 4($ respectively if $p \equiv 1 \bmod 4)$. This congruence information implies that $\left(\left\|\tilde{\varepsilon}_{0}(\mathcal{X})\right\|_{p} \cdot \tilde{\varepsilon}_{0}(\mathcal{X})\right)(\psi)$ is a square in $\mathbb{Z}_{p}^{*}$. This leads to a direct check of part (a) of Proposition 7.3 and of Theorem 7.11 in this case.

\section{ACKNOWLEDGEMENTS}

We are grateful to Boas Erez for urging us to clarify the relationship between Pfaffians and signatures. We also wish to thank the referee for improving this paper with many extraordinarily helpful comments and suggestions.

\section{Appendix A. Comparison of definitions}

The symplectic hermitian class group that we have used, namely $\mathrm{H}^{\mathrm{s}}(\mathbb{Z}[G])$, is very well suited to comparison with Arakelov invariants; indeed, from (5.6) we see that it is the natural vehicle for carrying discriminantal signs associated to Arakelov discriminants. In this appendix we briefly indicate how the class group $\mathrm{H}^{\mathrm{s}}(\mathbb{Z}[G])$, and hermitian classes formed in this group, relate to the previous hermitian classes and class groups, such as those used for instance in [Frö84] and [CPT03]. 


\section{Pfaffians AND Hodge DiscRiminants}

Recall that $\mathrm{H}^{\mathrm{s}}(\mathbb{Z}[G])$ was defined in Definition 5.1. By contrast in [Frö84] and [CPT03] the hermitian class group $\mathrm{HCl}(\mathbb{Z}[G])$ is used, which is described in terms of character functions as

$$
\operatorname{HCl}(\mathbb{Z}[G])=\frac{\operatorname{Hom}_{\Omega_{\mathbb{Q}}}\left(R_{G}, J_{f}\right) \times \operatorname{Det}\left(\mathbb{R}[G]^{\times}\right) \times \operatorname{Hom}_{\Omega_{\mathbb{Q}}}\left(R_{G}^{\mathrm{s}}, \overline{\mathbb{Q}}^{\times}\right)}{\operatorname{Im}(\widetilde{\Delta}) \cdot\left(\operatorname{Det}\left(\widehat{\mathbb{Z}}[G]^{\times} \times \mathbb{R}[G]^{\times}\right) \times 1\right)},
$$

where $\widetilde{\Delta}$ is the twisted diagonal map

$$
\widetilde{\Delta}: \operatorname{Det}\left(\mathbb{Q}[G]^{\times}\right) \rightarrow \operatorname{Hom}_{\Omega_{\mathbb{Q}}}\left(R_{G}, J_{f}\right) \times \operatorname{Det}\left(\mathbb{R}[G]^{\times}\right) \times \operatorname{Hom}_{\Omega_{\mathbb{Q}}}\left(R_{G}^{\mathrm{s}}, \overline{\mathbb{Q}}^{\times}\right)
$$

given by $\widetilde{\Delta}(\operatorname{Det}(a))=\operatorname{Det}(a) \times \operatorname{Det}(a) \times \operatorname{Det}^{\mathrm{s}}(a)^{-1}$. Comparing (5.3) with (A.1) it follows that there is a natural map

induced by the map

$$
\phi: \operatorname{HCl}(\mathbb{Z}[G]) \rightarrow \mathrm{H}^{\mathrm{s}}(\mathbb{Z}[G])
$$

$$
\operatorname{Hom}_{\Omega_{\mathbb{Q}}}\left(R_{G}, J_{f}\right) \times \operatorname{Det}\left(\mathbb{R}[G]^{\times}\right) \times \operatorname{Hom}_{\Omega_{\mathbb{Q}}}\left(R_{G}^{\mathrm{s}}, \overline{\mathbb{Q}}^{\times}\right) \rightarrow \operatorname{Hom}_{\Omega_{\mathbb{Q}}}\left(R_{G}^{\mathrm{s}}, J_{f}\right) \times \operatorname{Hom}\left(R_{G}^{\mathrm{s}}, \mathbb{R}^{\times}\right)
$$

which takes the first left-hand factor into the first right-hand factor by restriction from $R_{G}$ to $R_{G}^{\mathrm{s}}$; which is trivial on the second left-hand factor; and which maps the third left-hand factor to the second right-hand factor by inverting the natural map induced by the inclusion $\overline{\mathbb{Q}} \hookrightarrow \mathbb{C}$.

\section{REFERENCES}

AS68 M. F. Atiyah and I. Singer, The index of elliptic operators III, Ann. of Math. (2) 87 (1968), $564-604$.

Bis95 J. M. Bismut, Equivariant immersions and Quillen metrics, J. Differential Geom. 41 (1995), $53-157$.

CNT83 Ph. Cassou-Noguès and M. J. Taylor, Local root numbers and hermitian Galois structure of rings of integers, Math. Ann. 263 (1983), 251-261.

CEPT96 T. Chinburg, B. Erez, G. Pappas and M. J. Taylor, Tame actions of group schemes: integrals and slices, Duke Math. J. 82 (1996), 269-308.

CEPT97 T. Chinburg, B. Erez, G. Pappas and M. J. Taylor, E-constants and Galois structure of de Rham cohomology, Ann. of Math. (2) 146 (1997), 411-473.

CEPT98 T. Chinburg, B. Erez, G. Pappas and M. J. Taylor, On the $\varepsilon$-constants of arithmetic schemes, Math. Ann. 311 (1998), 377-395.

CPT02 T. Chinburg, G. Pappas and M. J. Taylor, e-constants and equivariant Arakelov Euler characteristics, Ann. Sci. École Norm. Sup. 35 (2002), 307-352.

CPT03 T. Chinburg, G. Pappas and M. J. Taylor, Duality and hermitian Galois module structure, Proc. London Math. Soc. 87 (2003), 54-108.

CPT07 T. Chinburg, G. Pappas and M. J. Taylor, Cubic structures, equivariant Euler characteristics and lattices of modular forms, Ann. of Math (2), to appear.

Del74 P. Deligne, Les constantes des équations fonctionelles de la fonction L, Lecture Notes in Mathematics, vol. 349 (Springer, Berlin, 1974), 501-597.

Del79 P. Deligne, Valeurs de fonctions L et périodes d'intégrales, Proc. Symp. Pure Math. 33 (1979), 313-346.

DP61 A. Dold and D. Puppe, Homologie nicht-additiver Funktoren. Anwendungen, Ann. Inst. Fourier 11 (1961), 201-312.

Frö83 A. Fröhlich, Galois module structure of algebraic integers, Springer Ergebnisse, 3 Folge, Band 1 (Springer, Berlin, 1983).

Frö84 A. Fröhlich, Classgroups and hermitian modules, Progress in Mathematics, vol. 48 (Birkhäuser, Basel, 1984).

Ful98 W. Fulton, Intersection theory, second edition (Springer, Berlin, 1998). 


\section{Pfaffians AND Hodge DiscRiminants}

GSZ91 H. Gillet and C. Soulé, with an appendix by D. Zagier, Analytic torsion and the arithmetic Todd genus, Topology 30 (1991), 21-54.

GH78 P. Griffiths and J. Harris, Principles of algebraic geometry (Wiley, New York, 1978).

Gro66 A. Grothendieck, On the de Rham cohomology of algebraic varieties, Publ. Math. Inst. Hautes Études Sci. 29 (1966), 95-103.

Har77 R. Hartshorne, Algebraic geometry, Graduate Texts in Mathematics, vol. 52 (Springer, Berlin, 1977).

Ill71 L. Illusie, Complexe cotangent et déformations, Lecture Notes in Mathematics, vol. 239 (Springer, Berlin, 1971).

Kat94 K. Kato, Class field theory, D-modules, and ramification on higher dimensional schemes, part 1, Amer. J. Math. 116 (1994), 757-784.

KM76 F. Knudsen and D. Mumford, The projectivity of the moduli space of stable curves. I. Preliminaries on "det" and "Div", Math. Scand. 39 (1976), 19-55.

Lan84 S. Lang, Algebra, second edition (Addison-Wesley, Reading, MA, 1984).

MR04 V. Maillot and D. Roessler, On the periods of motives with complex multiplication and a conjecture of Gross-Deligne, Ann. of Math. (2) 160 (2004), 727-754.

RS73 D. B. Ray and I. M. Singer, Analytic torsion for complex manifolds, Ann. of Math. (2) 98 (1973), 154-177.

Rob98 P. Roberts, Multiplicities and Chern classes in local algebra, Cambridge Tracts in Mathematics, vol. 133 (Cambridge University Press, Cambridge, 1998).

Sai93 T. Saito, $\epsilon$-factor of tamely ramified sheaf on a variety, Invent. Math. 113 (1993), 389-417.

Ser86 J. P. Serre, Linear representations of finite groups, third edition (Springer, Berlin, 1986).

Sha78 P. Shanahan, The Atiyah-Singer index theorem, Lecture Notes in Mathematics, vol. 638 (Springer, Berlin, 1978).

SABK92 C. Soulé, D. Abramovich, J.-F. Burnol and J. Kramer, Lectures on Arakelov geometry, Cambridge Studies in Advanced Mathematics, vol. 33 (Cambridge University Press, Cambridge, 1992).

Tay84 M. J. Taylor, Classgroups of group rings, London Mathematical Society Lecture Note Series, vol. 91 (Cambridge University Press, Cambridge, 1984).

Ver73 J. L. Verdier, Caractéristique d'Euler-Poincaré, Bull. Soc. Math. France 101 (1973), 447-448.

Ted Chinburg ted@math.upenn.edu

Department of Mathematics, University of Pennsylvania, Philadelphia, PA 19104, USA

Georgios Pappas pappas@math.msu.edu

Michigan State University, East Lansing, MI 48824, USA

Martin Taylor martin.taylor@manchester.ac.uk

Department of Mathematics, University of Manchester, Manchester M60 1QD, UK 